Supporting Information

to

\title{
Virtual cocrystal screening methods as tools to understand the formation of pharmaceutical co-crystals - A case study of linezolid, a wide-range antibacterial drug
}

\author{
Mehrnaz Khalaji, Marek J. Potrzebowski and Marta K. Dudek*
}

Centre of Molecular and Macromolecular Studies of Polish Academy of Sciences, Sienkiewicza 112, 90-363 Lodz, Poland

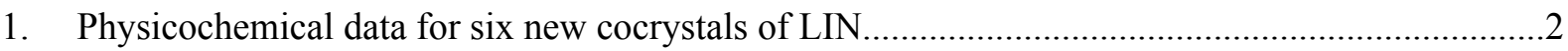

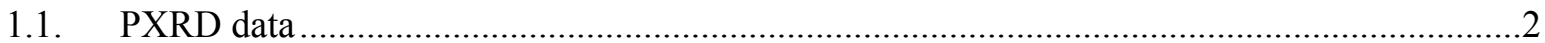

1.2. Comparison of the PXRD data for the new cocrystals of LIN with the simulated PXRD

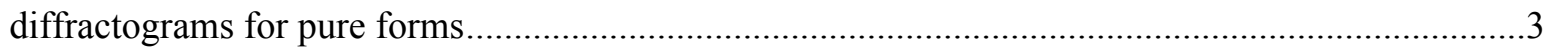

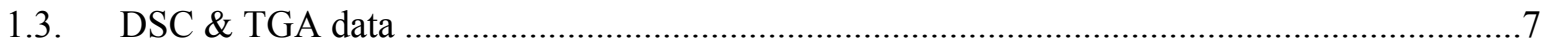

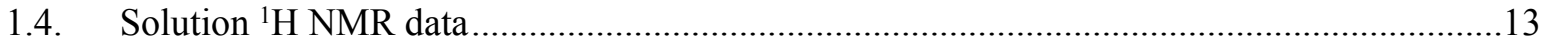

2. Solid-state NMR spectra registered for pure components and the reaction mixtures obtained after

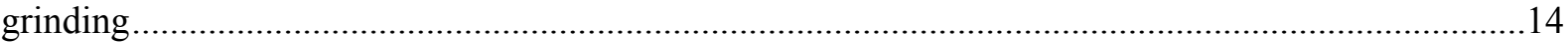

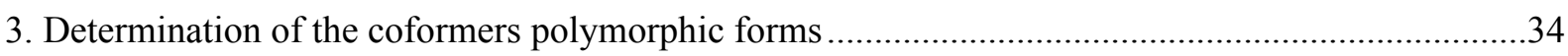

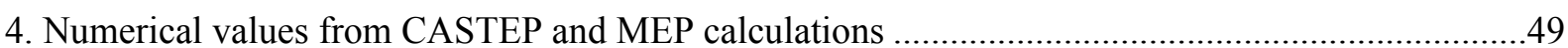

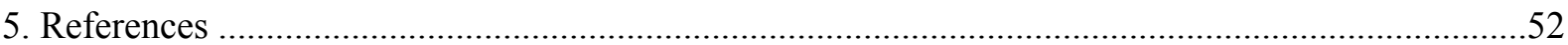




\section{Physicochemical data for six new cocrystals of LIN}

\subsection{PXRD data}
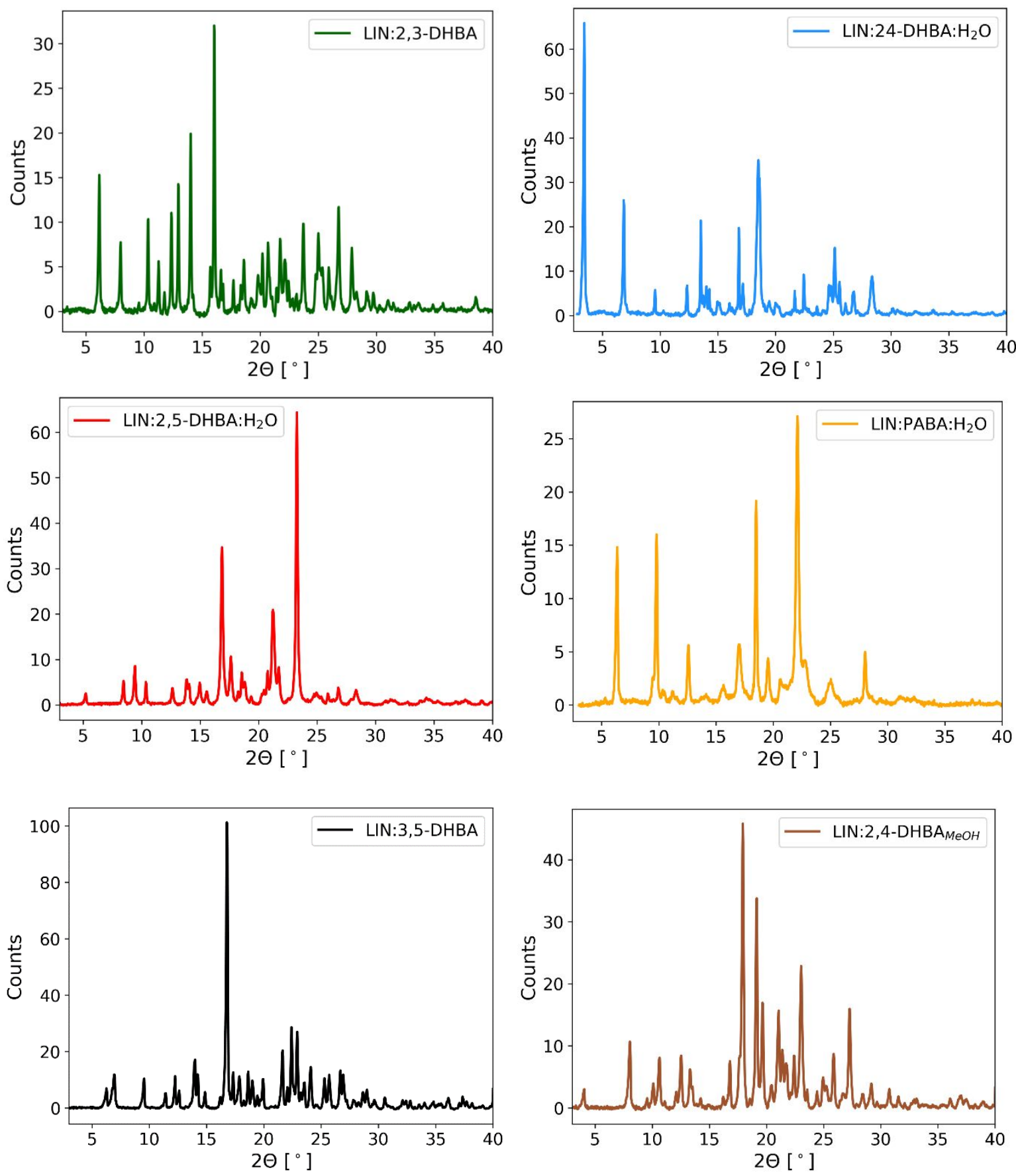

Figures S1. PXRD data for six new crystal phases of LIN with 2,3-DHBA, 2,4-DHBA, 3,5DHBA, 2,4-DHBA and water, 2,5-DHBA and water, and PABA and water. 


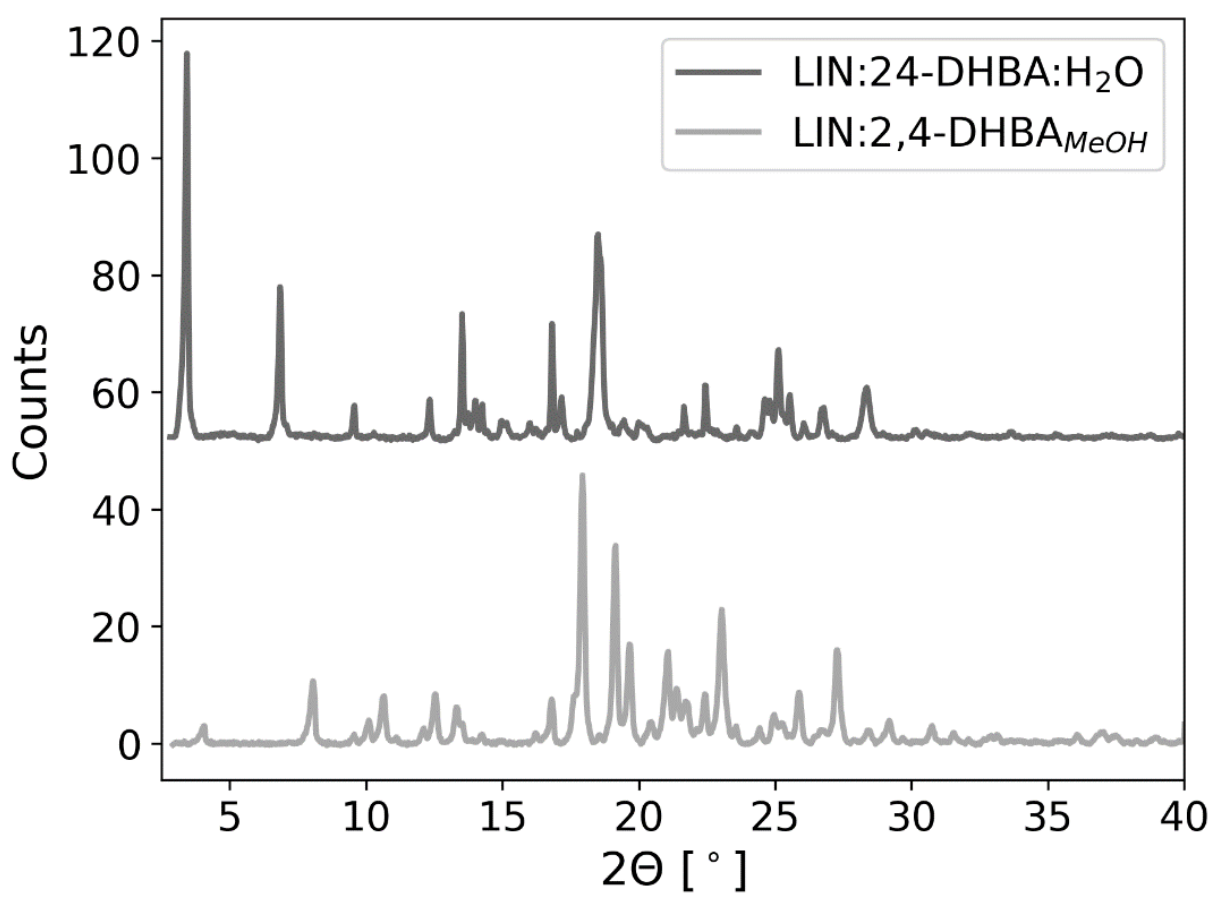

Figure S2. Comparison of PXRD data for LIN:2,4-DHBA and LIN:2,4-DHBA: $\mathrm{H}_{2} \mathrm{O}$ crystals

1.2. Comparison of the PXRD data for the new cocrystals of LIN with the simulated PXRD diffractograms for pure forms

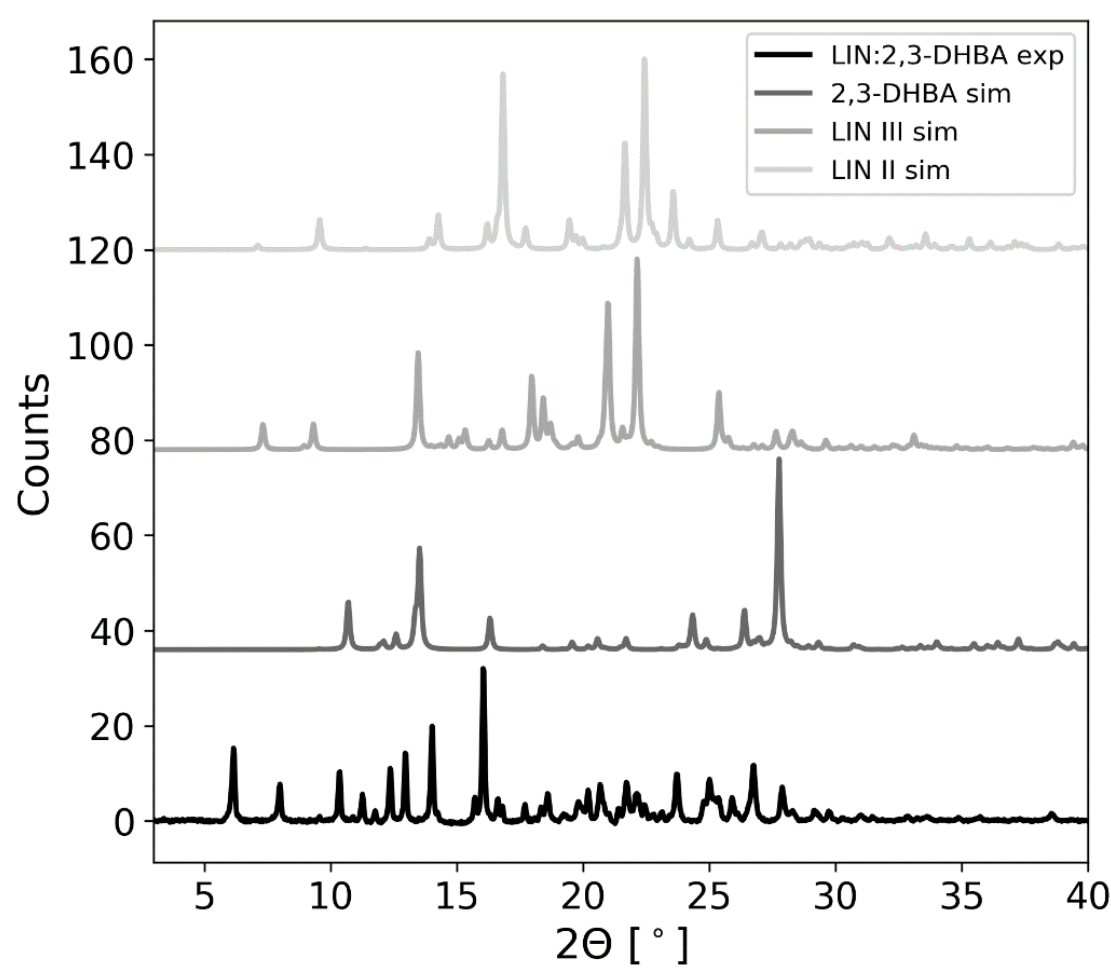

Figure S3. A comparison of simulated PXRD for pure LIN forms II and III and 2,3-DHBA triclinic form with the experimental PXRD for LIN:2,3-DHBA cocrystal 


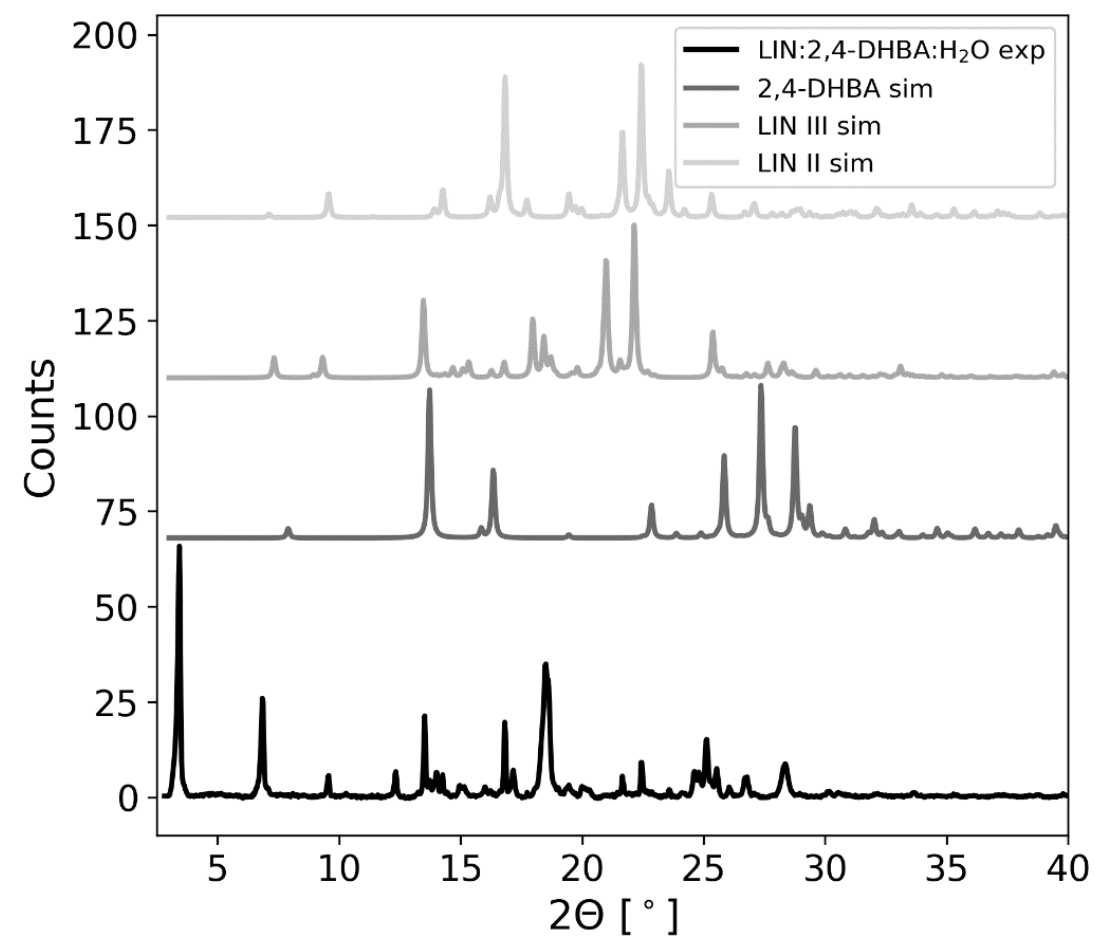

Figure S4. A comparison of simulated PXRD for pure LIN forms II and III and 2,4-DHBA form II with the experimental PXRD for LIN:2,4-DHBA cocrystal hydrate.

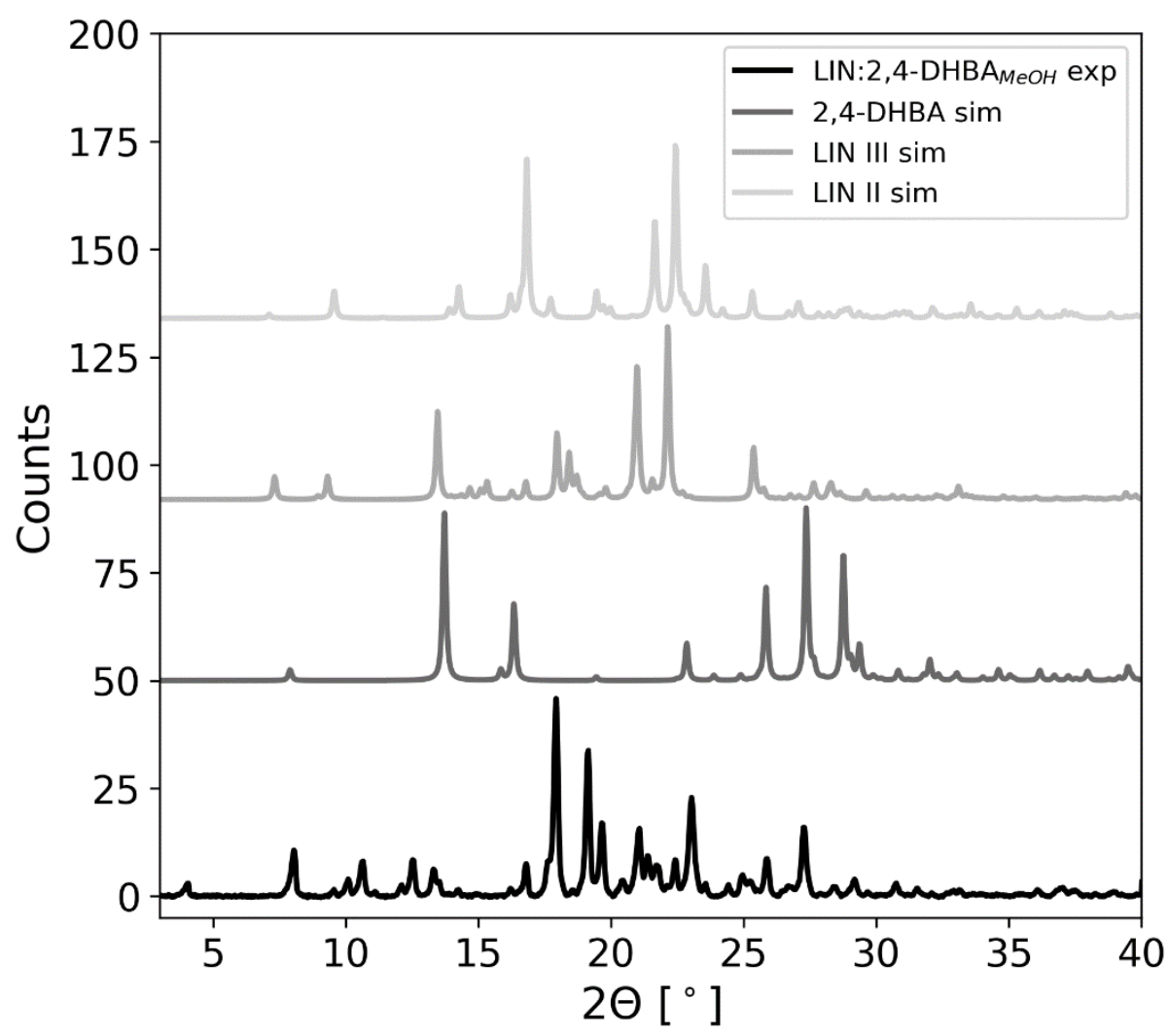

Figure S5. A comparison of simulated PXRD for pure LIN forms II and III and 2,4-DHBA form II with the experimental PXRD for LIN:2,4-DHBA cocrystal. 


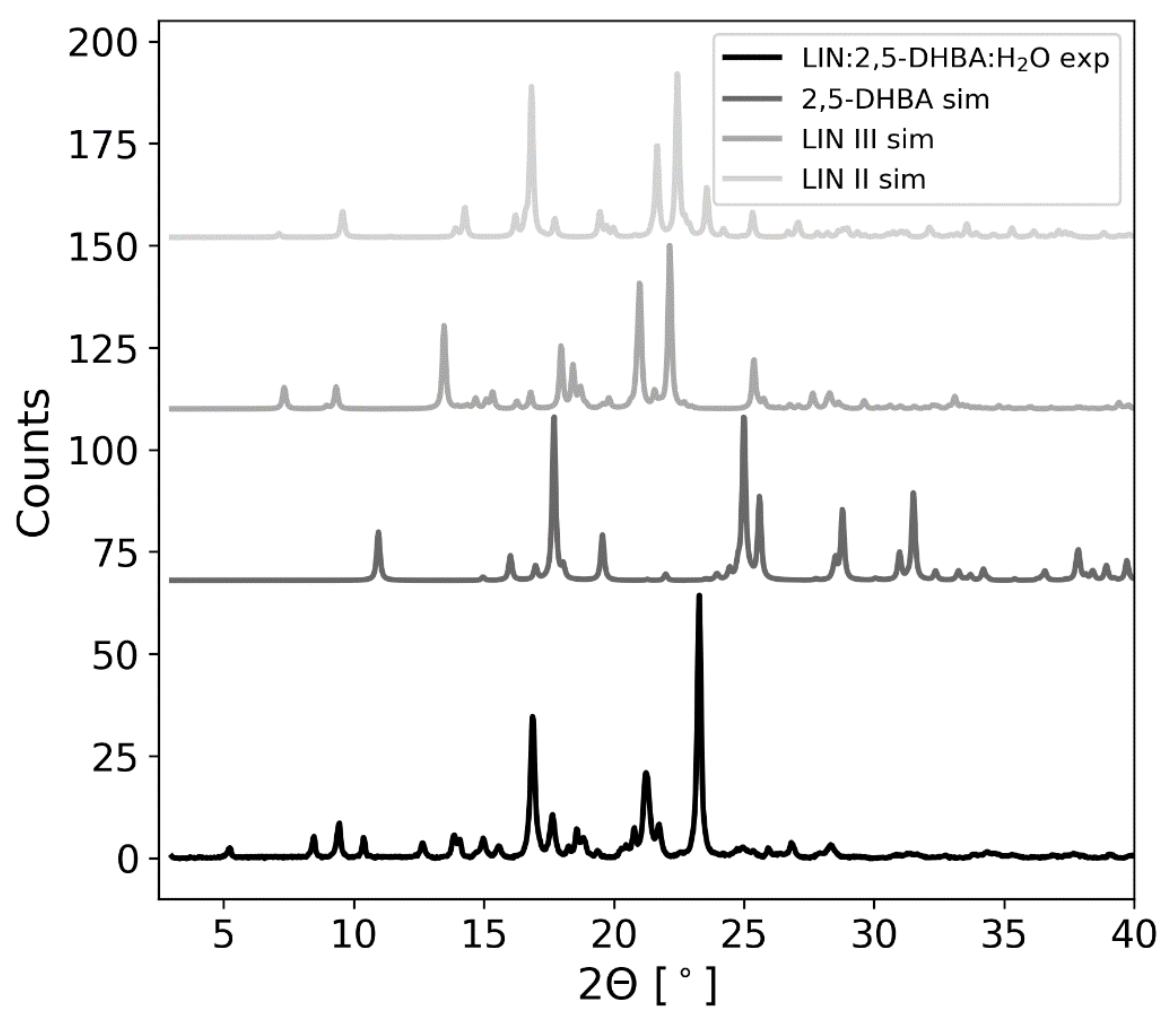

Figure S6. A comparison of simulated PXRD for pure LIN forms II and III and 2,5-DHBA form I with the experimental PXRD for LIN:2,5-DHBA cocrystal hydrate.

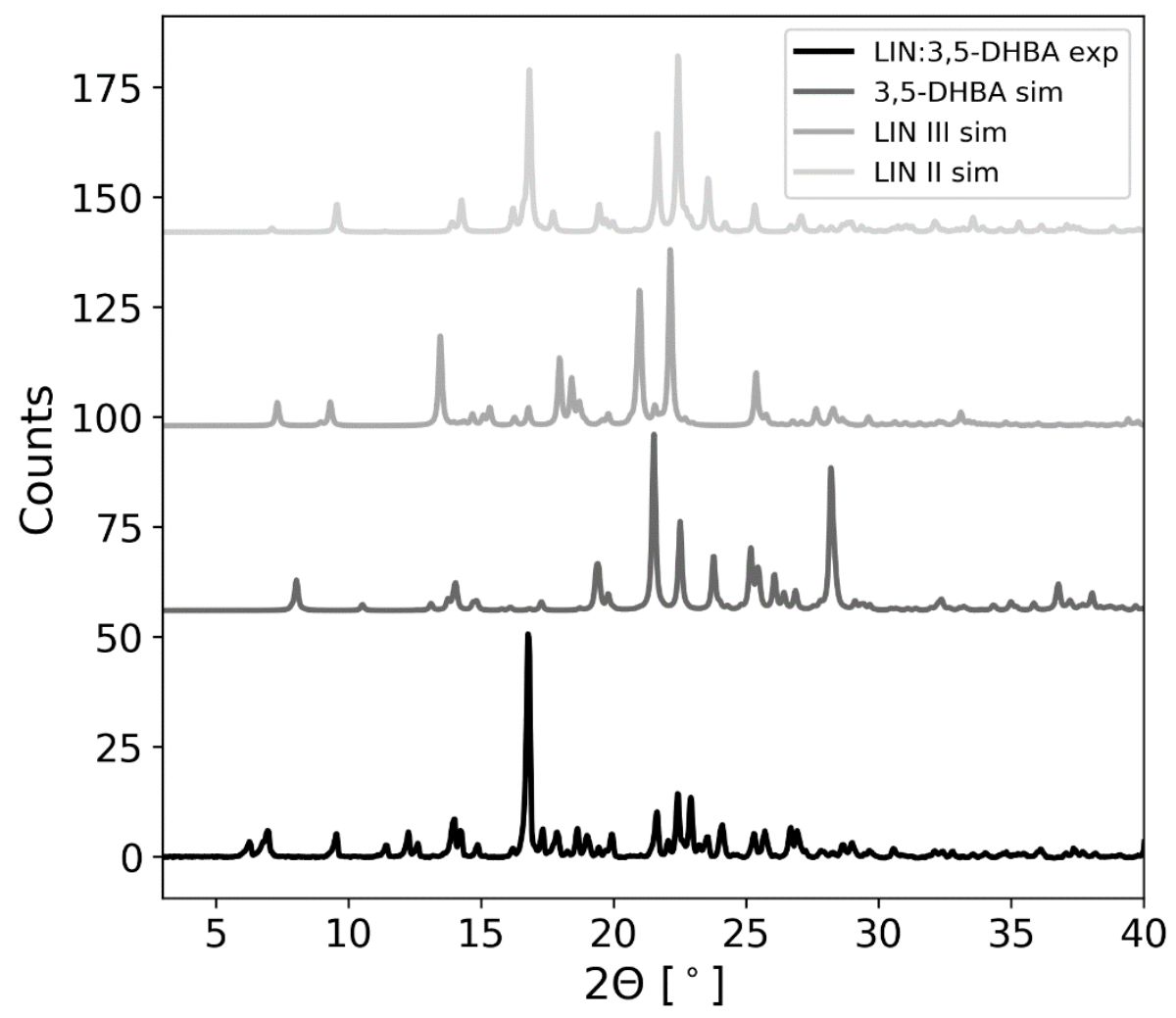

Figure S7. A comparison of simulated PXRD for pure LIN forms II and III and 3,5-DHBA form II with the experimental PXRD for LIN:3,5-DHBA cocrystal. 


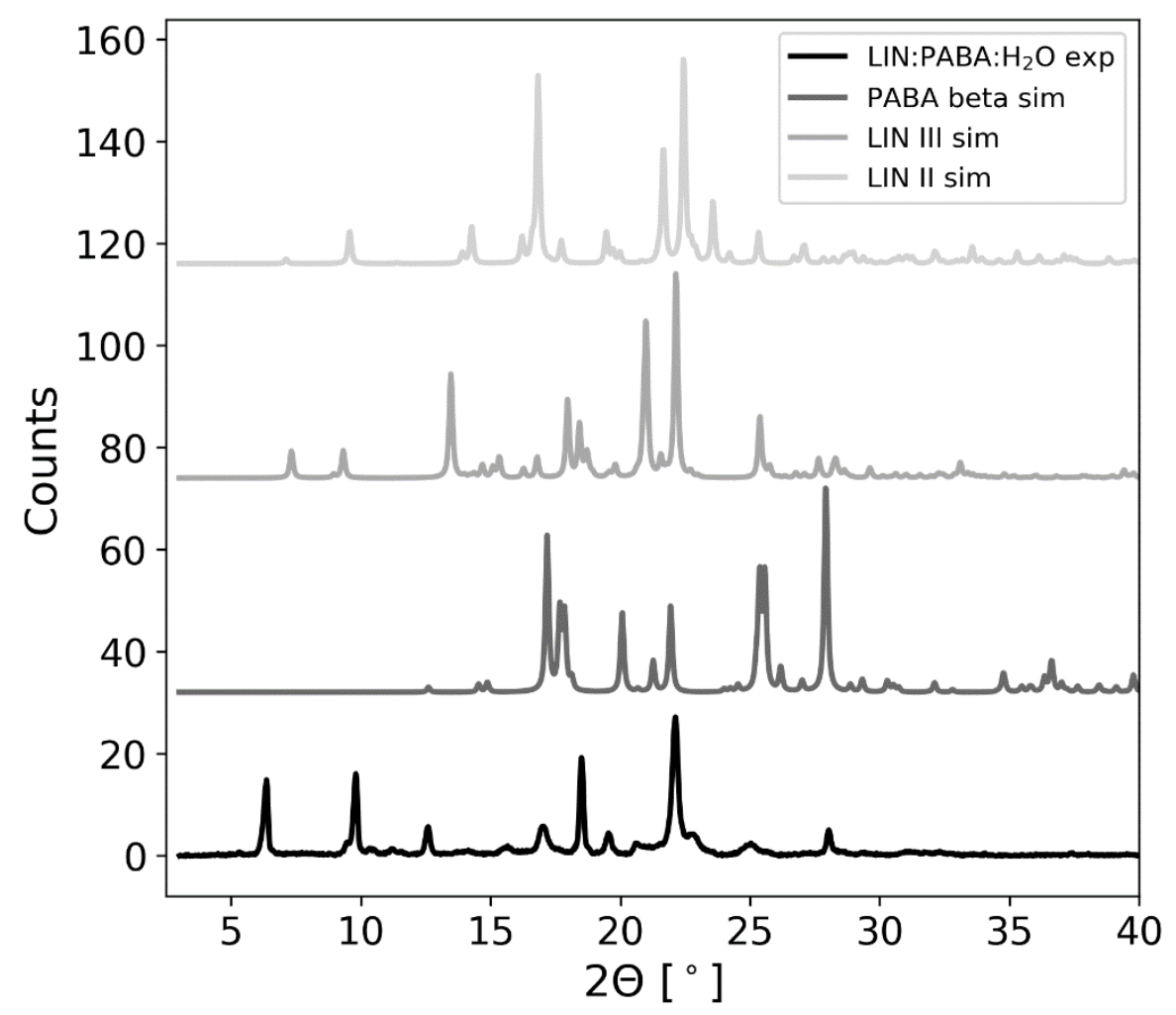

Figure S8. A comparison of simulated PXRD for pure LIN forms II and III and PABA form $\beta$ with the experimental PXRD for LIN:PABA cocrystal hydrate. 
1.3. DSC \& TGA data
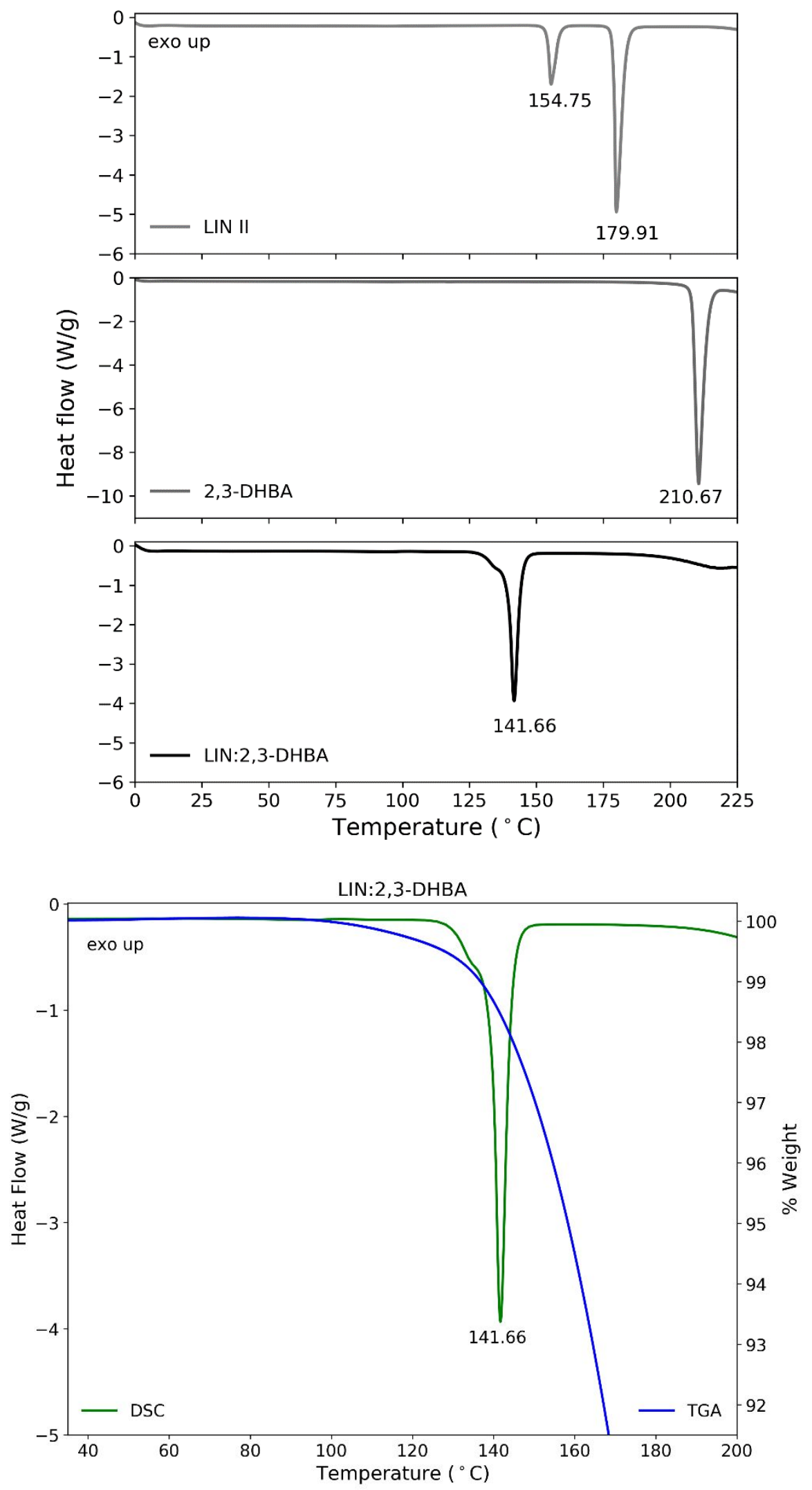

Figure S9. DSC thermograms for LIN:2,3-DHBA cocrystal, LIN form II and pure 2,3-DHBA, as well as TGA results for LIN:2,3-DHBA cocrystal. 

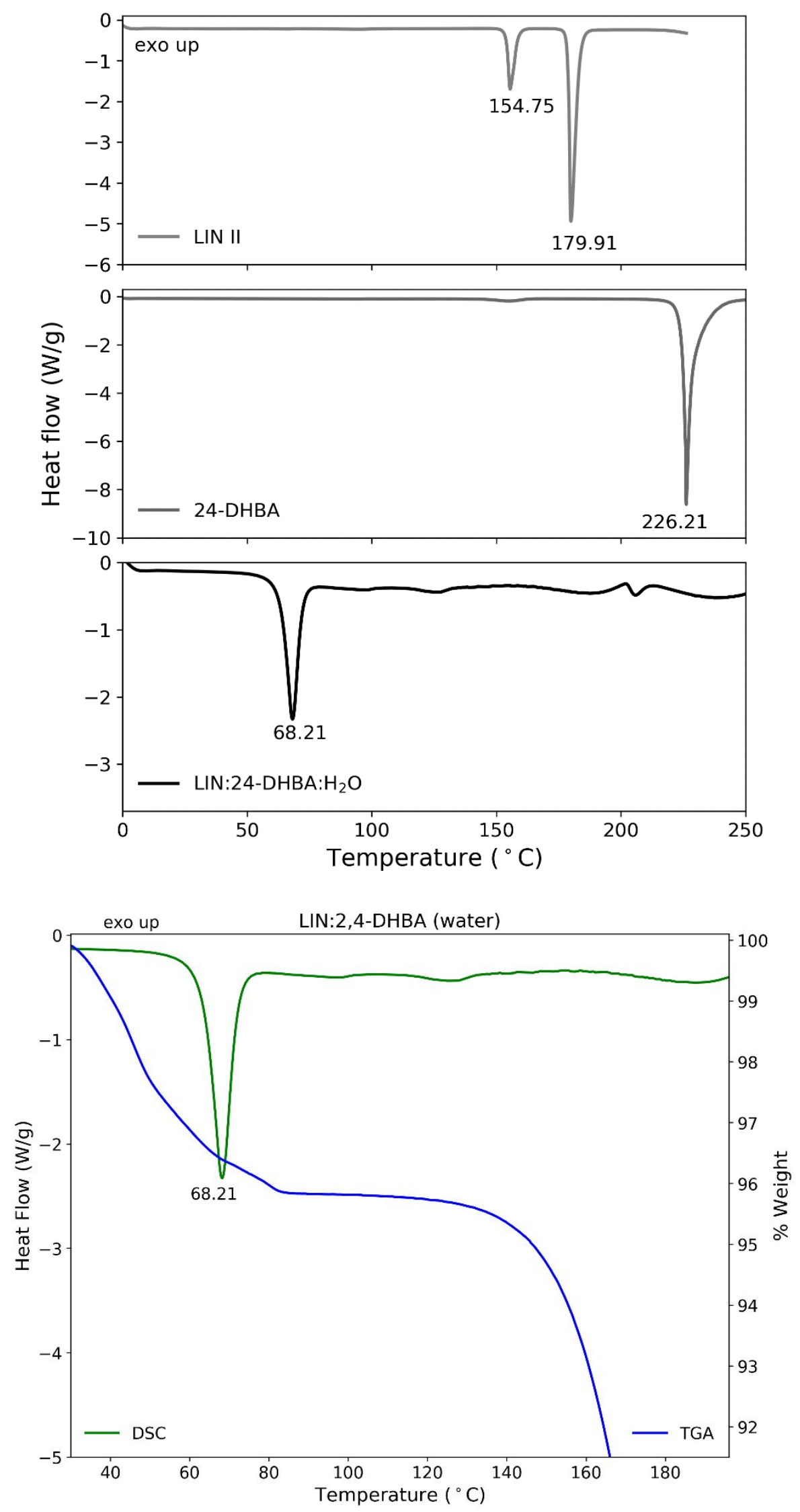

Figure S10. DSC thermograms for LIN:2,4-DHBA cocrystal hydrate, LIN form II and pure 2,4-DHBA, as well as TGA results for LIN:2,4-DHBA cocrystal hydrate. 

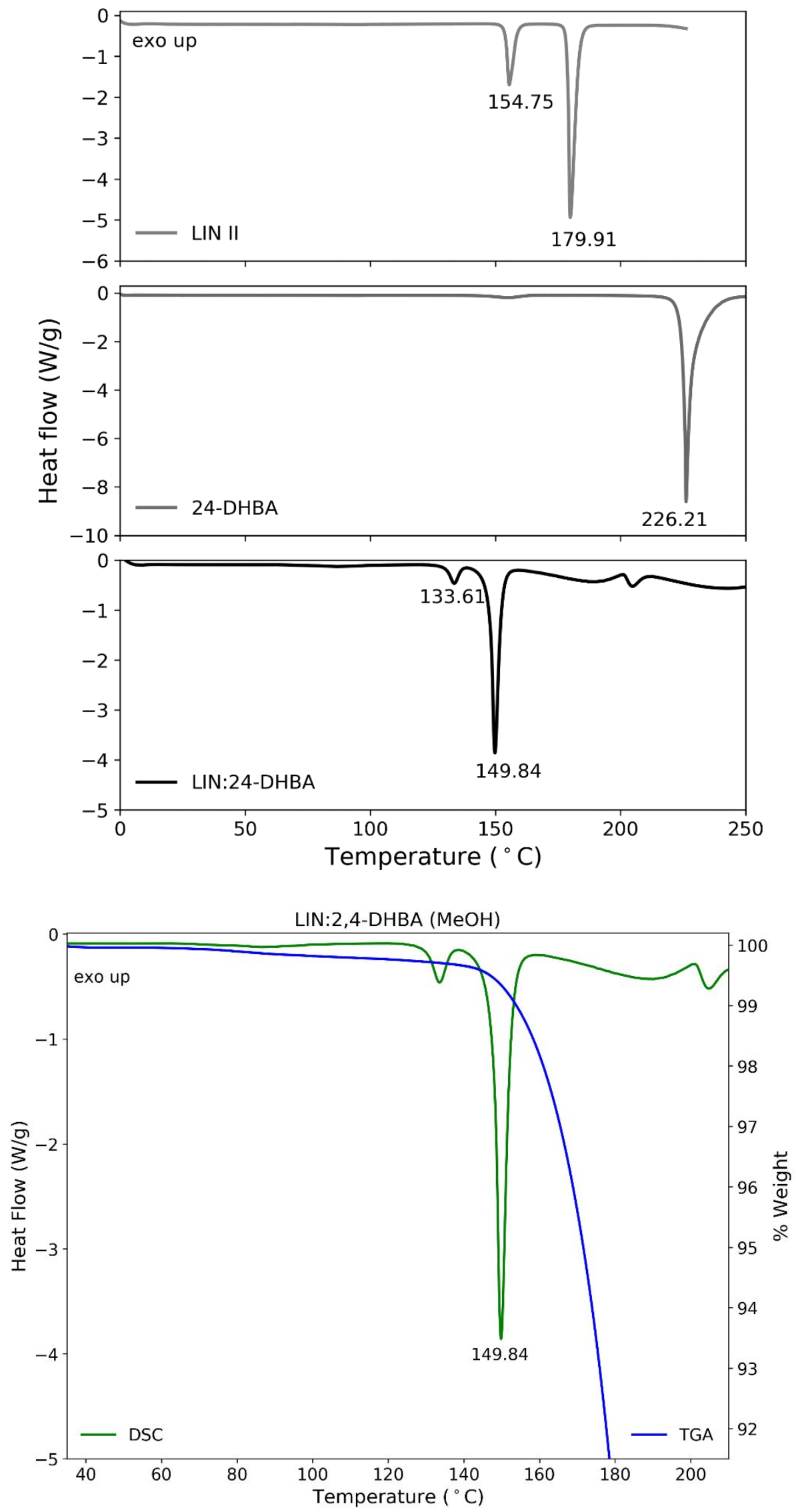

Figure S11. DSC thermograms for LIN:2,4-DHBA cocrystal, LIN form II and pure 2,4-DHBA, as well as TGA results for LIN:2,4-DHBA cocrystal. 

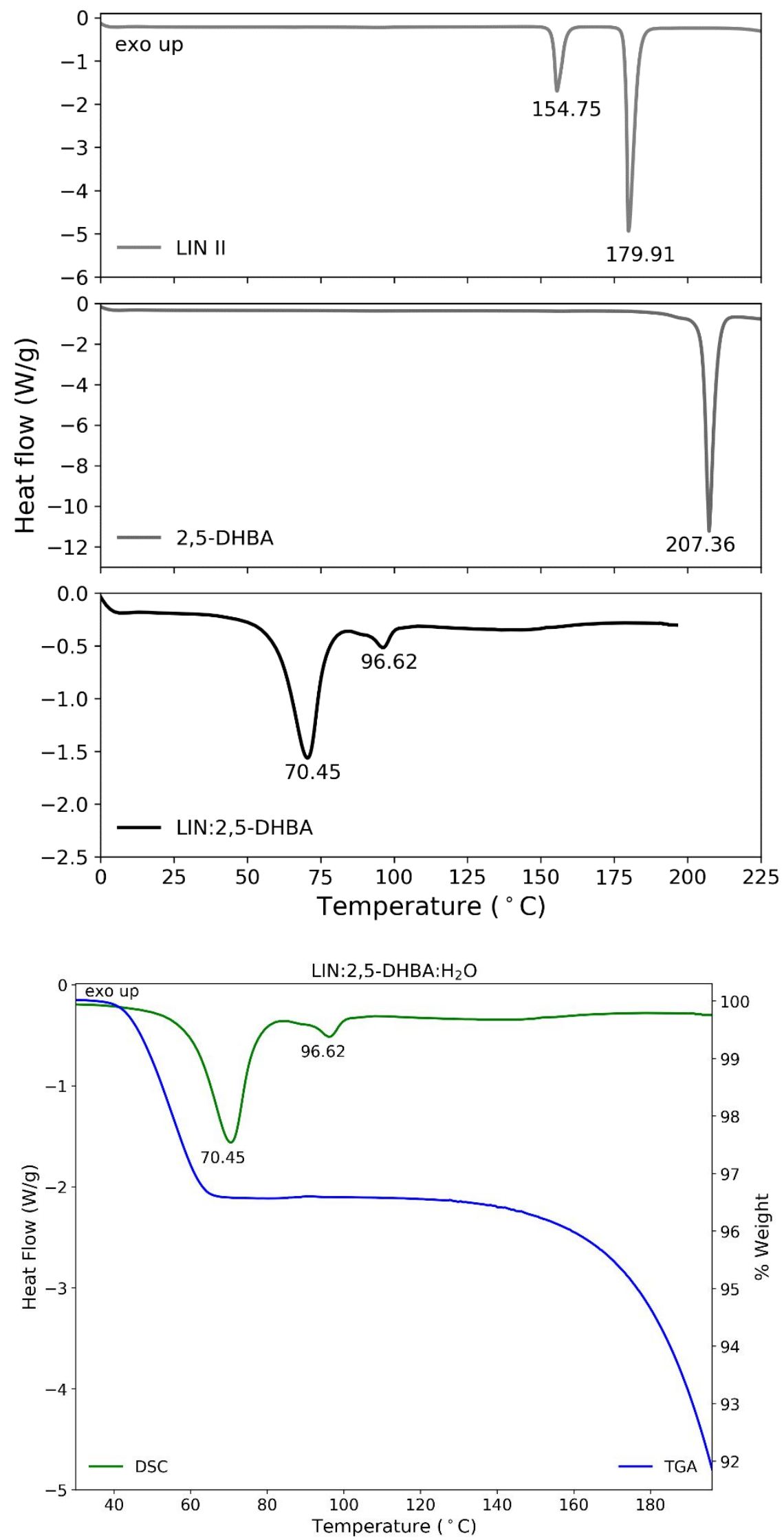

Figure S12. DSC thermograms for LIN:2,5-DHBA cocrystal hydrate, LIN form II and pure 2,5-DHBA, as well as TGA results for LIN:2,5-DHBA cocrystal hydrate. 

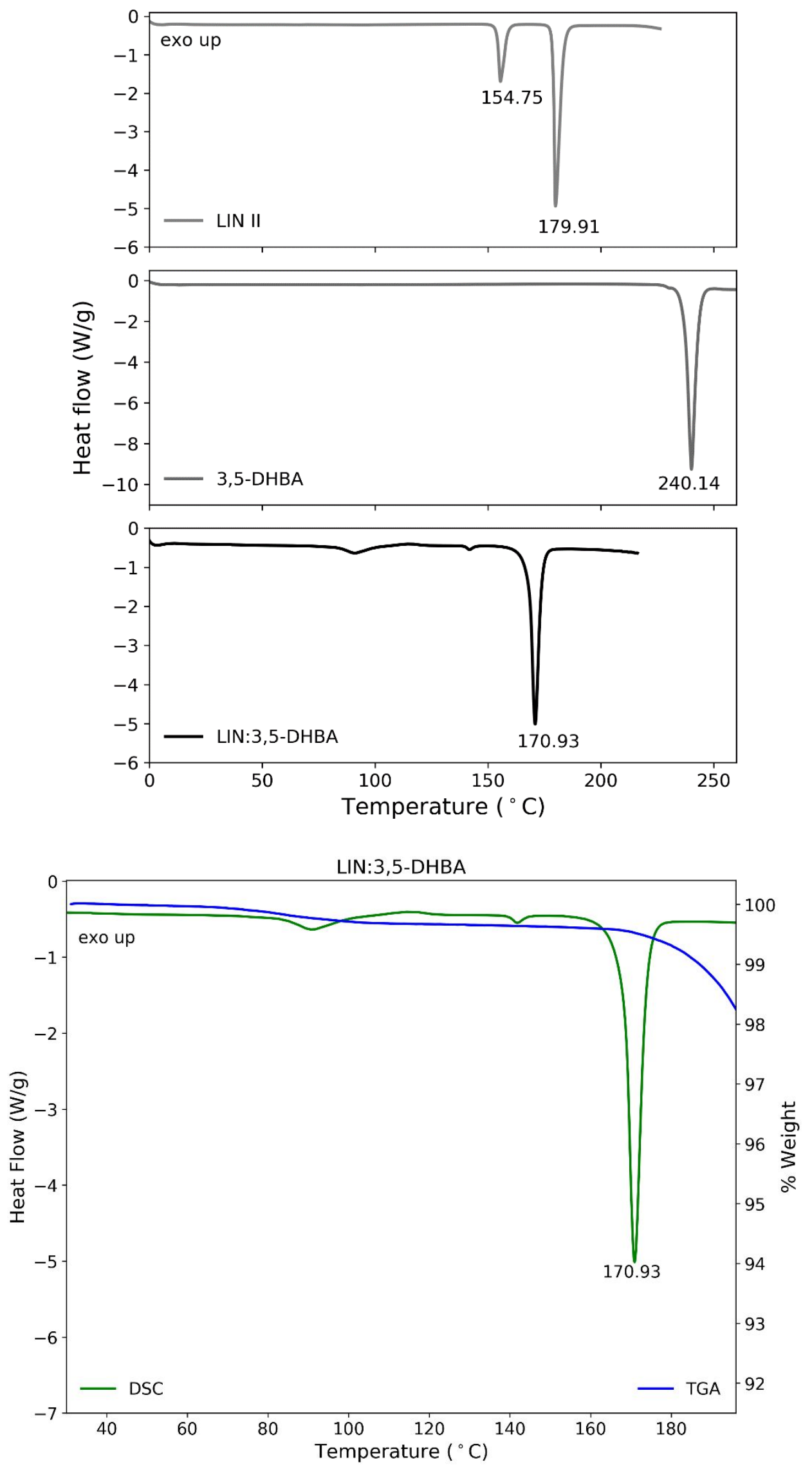

Figure S13. DSC thermograms for LIN:3,5-DHBA cocrystal, LIN form II and pure 3,5-DHBA, as well as TGA results for LIN:3,5-DHBA cocrystal. 

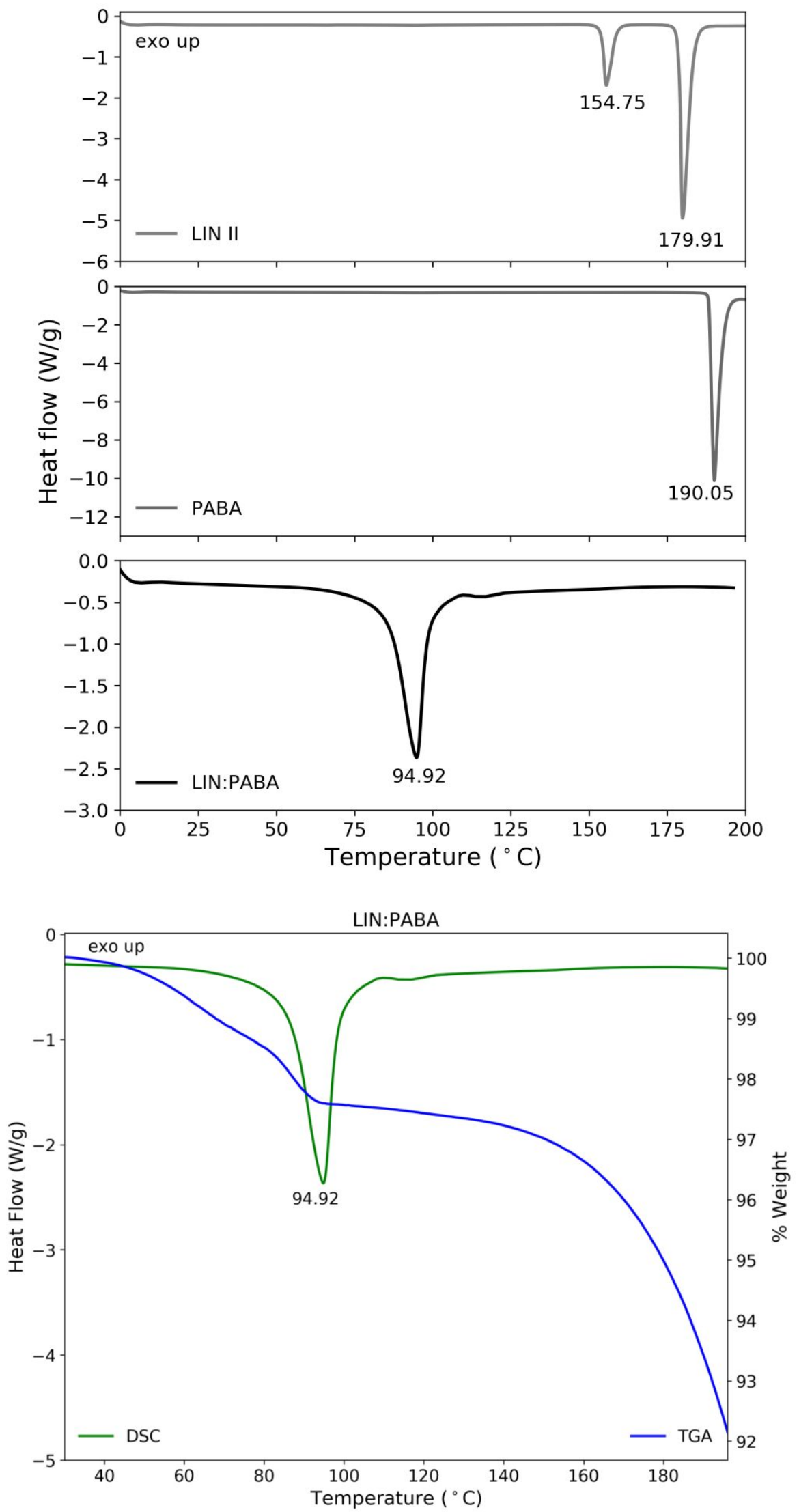

Figure S14. DSC thermograms for LIN:PABA cocrystal hydrate, LIN form II and pure PABA, as well as TGA results for LIN:PABA cocrystal hydrate. 


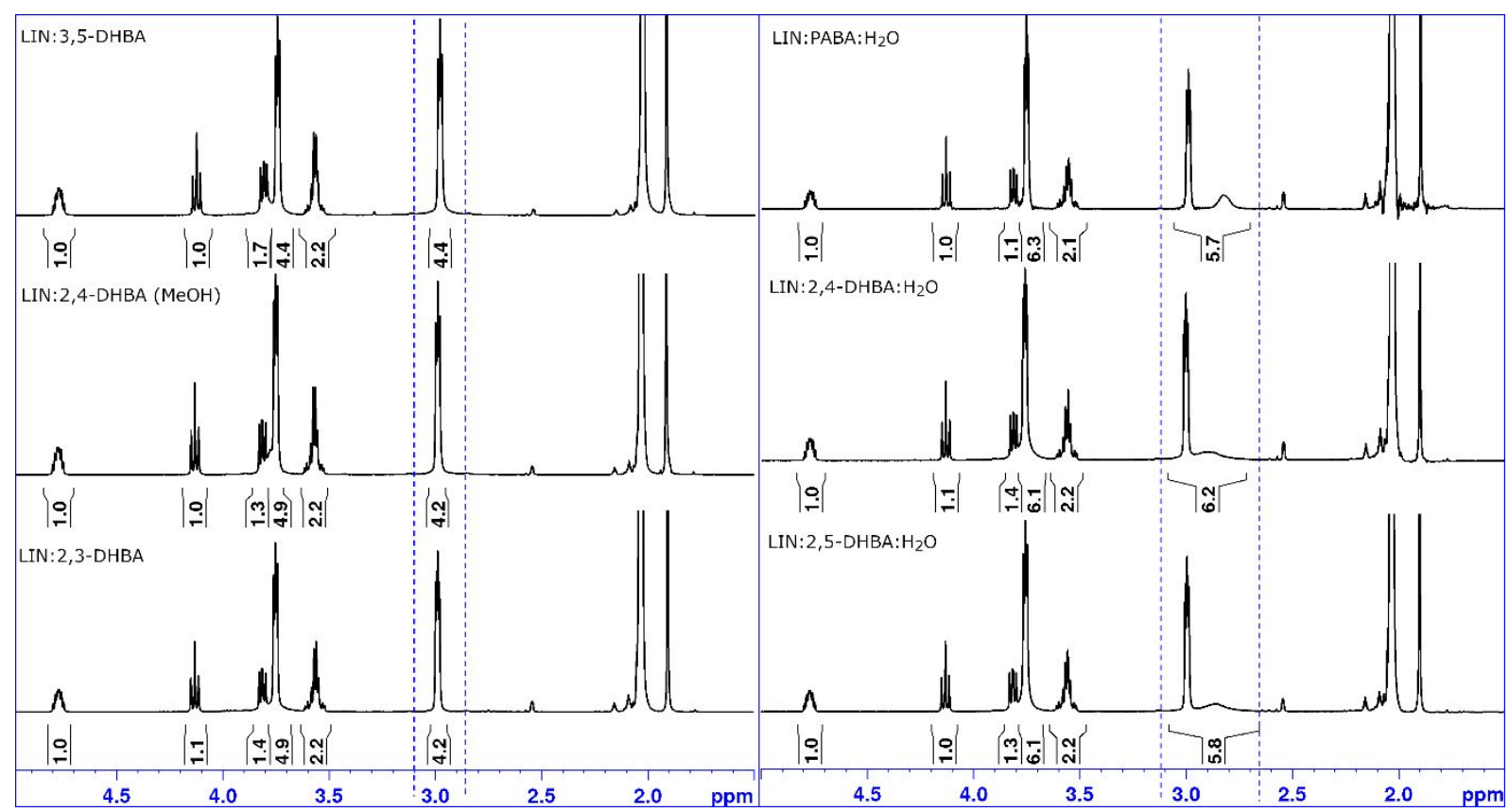

Figure S15. ${ }^{1} \mathrm{H}$ solution NMR spectra of the obtained cocrystals, registered in anhydrous acetone- $d_{6}$ in order to determine (if present) the amount of water present in the respective crystal structures. All ${ }^{1} \mathrm{H}$ signals were integrated in respect to a well-resolved LIN signal at $4.78 \mathrm{ppm}$. The left-hand side panel shows spectra of neat cocrystals, i.e. without the presence of water resonances, which is visible as a lack of ${ }^{1} \mathrm{H}$ resonances at ca. 2.8-2.9 ppm. Instead, only ${ }^{1} \mathrm{H}$ signals originating from 4 hydrogen atoms of LIN are visible (see a rectangle marked with the dashed lines at the left-hand side panel. The right-hand side panel shows ${ }^{1} \mathrm{H}$ NMR spectra for cocrystal hydrates - in these cases broadened ${ }^{1} \mathrm{H}$ resonances are visible at ca. 2.8-2.9 ppm, resulting in the integral value of ca. 2 . This suggest that in each case one molecule of water is present in each crystal lattice. 


\section{Solid-state NMR spectra registered for pure components and}

the reaction mixtures obtained after grinding

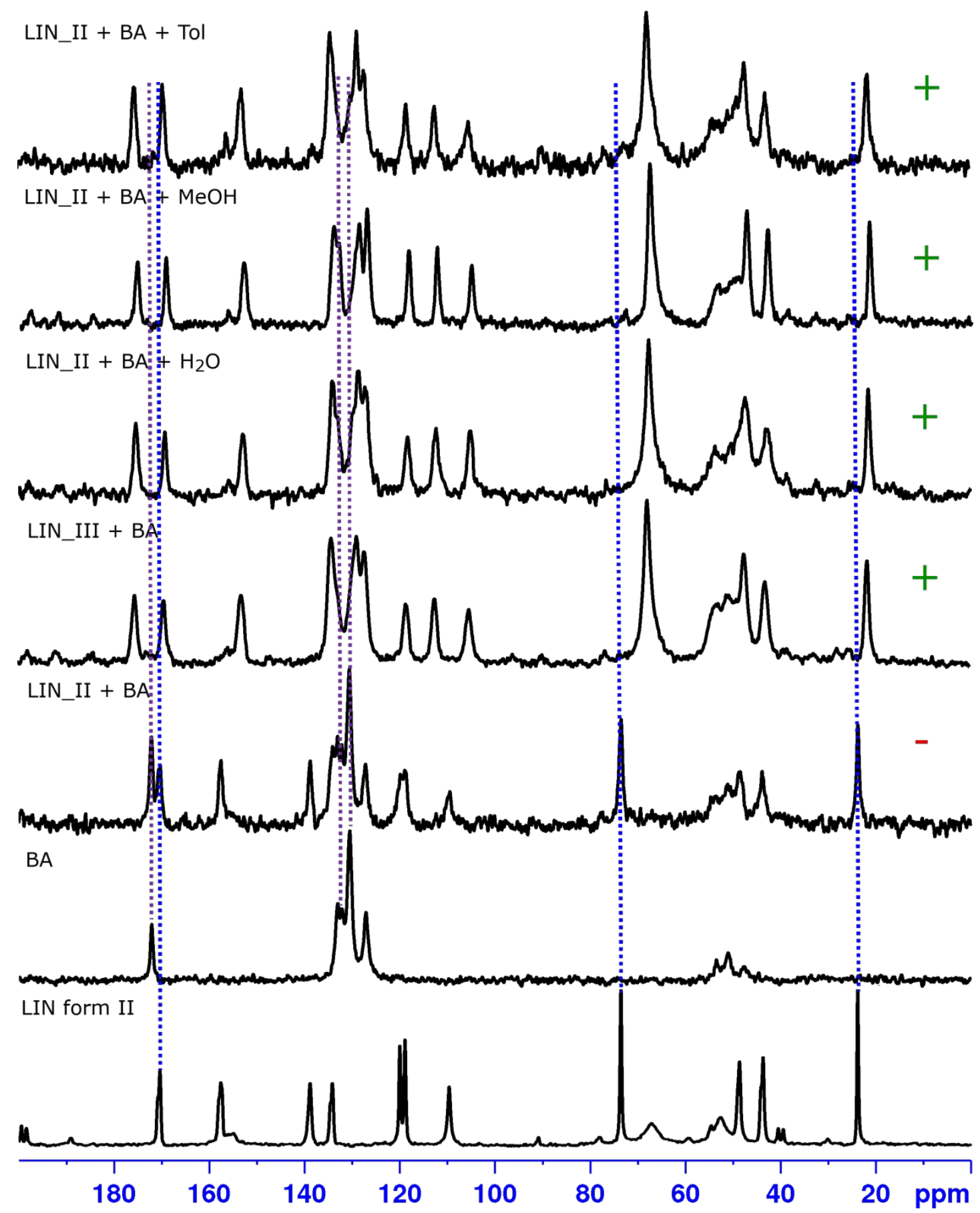

Figure S16. ${ }^{13} \mathrm{C}$ CPMAS NMR spectra for LIN form II, BA and various reaction mixtures obtained after grinding LIN_II or LIN_III with BA without any solvent and in the presence of water, methanol or toluene to create $\overline{L A G}^{-}$conditions. Dashed lines indicate the positions of ${ }^{13} \mathrm{C}$ signals originating from pure reagents, 'plus' or 'minus' signs indicate whether a cocrystal was formed in given reaction conditions. 


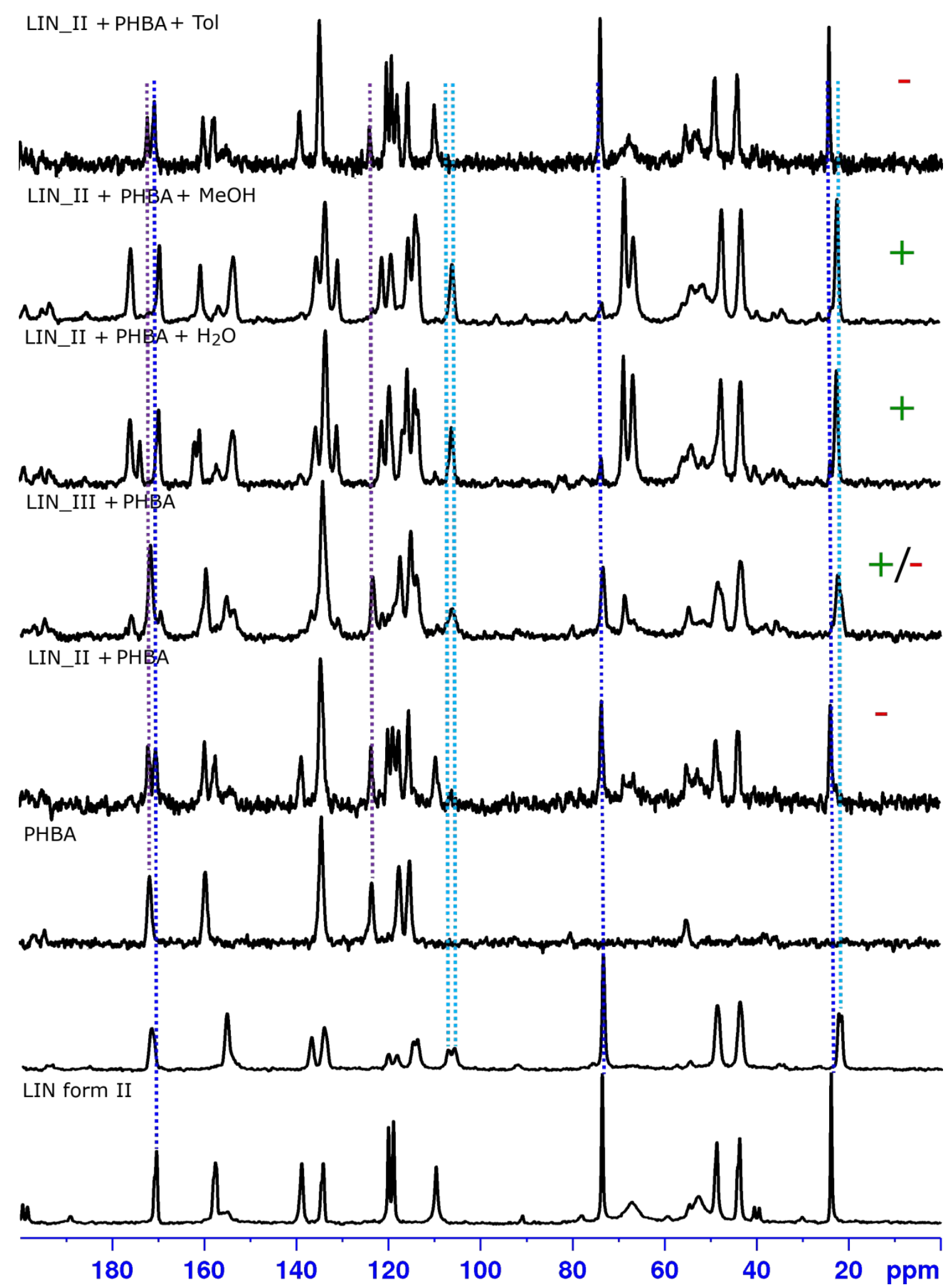

Figure S17. ${ }^{13} \mathrm{C}$ CPMAS NMR spectra for LIN form II, LIN form III, PHBA and various reaction mixtures obtained after grinding LIN_II or LIN_III with PHBA without any solvent and in the presence of water, methanol or toluene to create LAG conditions. Dashed lines indicate the positions of ${ }^{13} \mathrm{C}$ signals originating from pure reagents, 'plus' or 'minus' signs indicate whether a cocrystal was formed in given reaction conditions. 


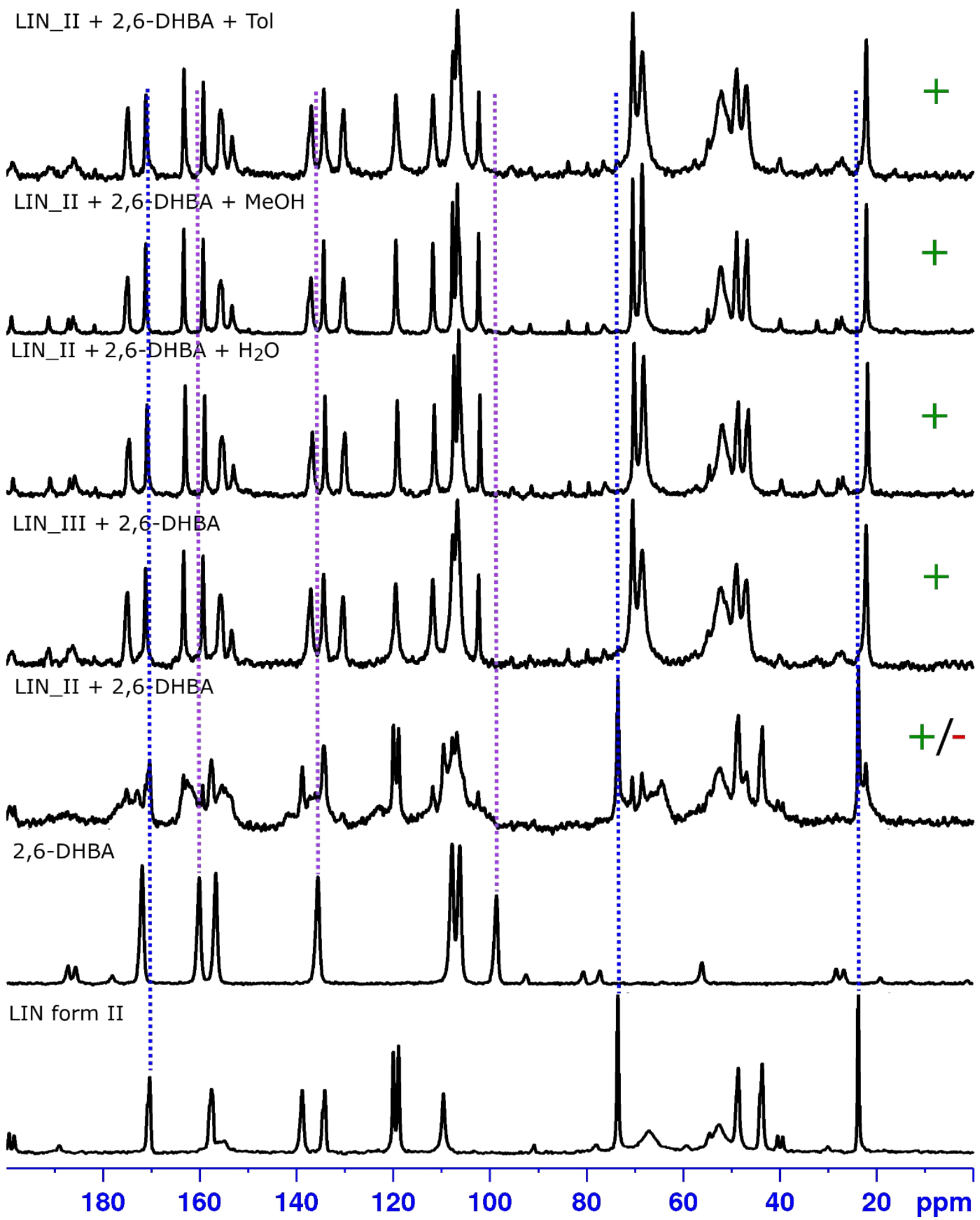

Figure S18. ${ }^{13} \mathrm{C}$ CPMAS NMR spectra for LIN form II, 2,6-DHBA and various reaction mixtures obtained after grinding LIN_II or LIN_III with 2,6-DHBA without any solvent and in the presence of water, methanol or toluene to create LAG conditions. Dashed lines indicate the positions of ${ }^{13} \mathrm{C}$ signals originating from pure reagents, 'plus' or 'minus' signs indicate whether a cocrystal was formed in given reaction conditions. 


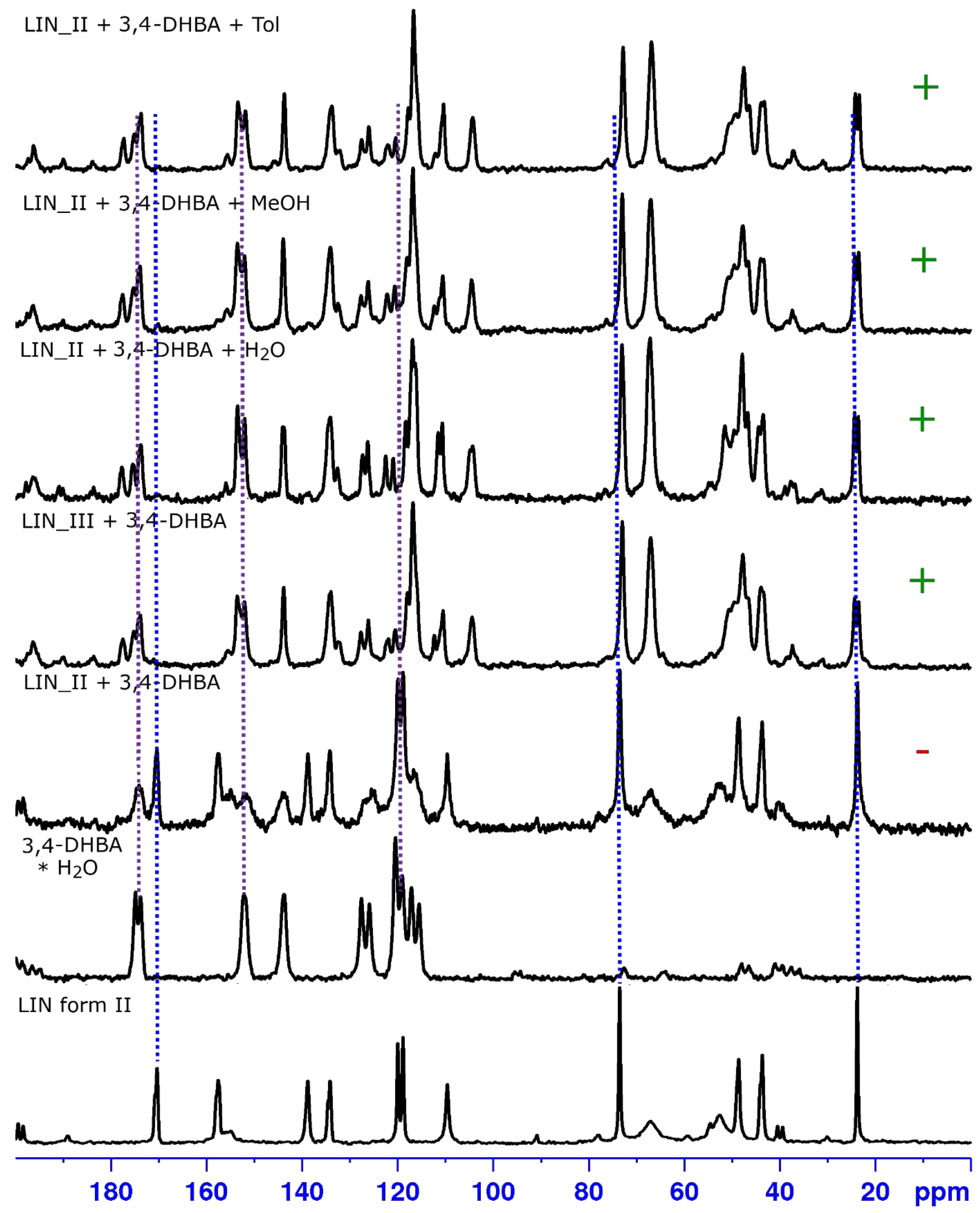

Figure S19. ${ }^{13} \mathrm{C}$ CPMAS NMR spectra for LIN form II, 3,4-DHBA* $\mathrm{H}_{2} \mathrm{O}$ and various reaction mixtures obtained after grinding LIN_II or LIN_III with 3,4-DHBA* $\mathrm{H}_{2} \mathrm{O}$ without any solvent and in the presence of water, methanol or toluene to create LAG conditions. Dashed lines indicate the positions of ${ }^{13} \mathrm{C}$ signals originating from pure reagents, 'plus' or 'minus' signs indicate whether a cocrystal was formed in given reaction conditions. 


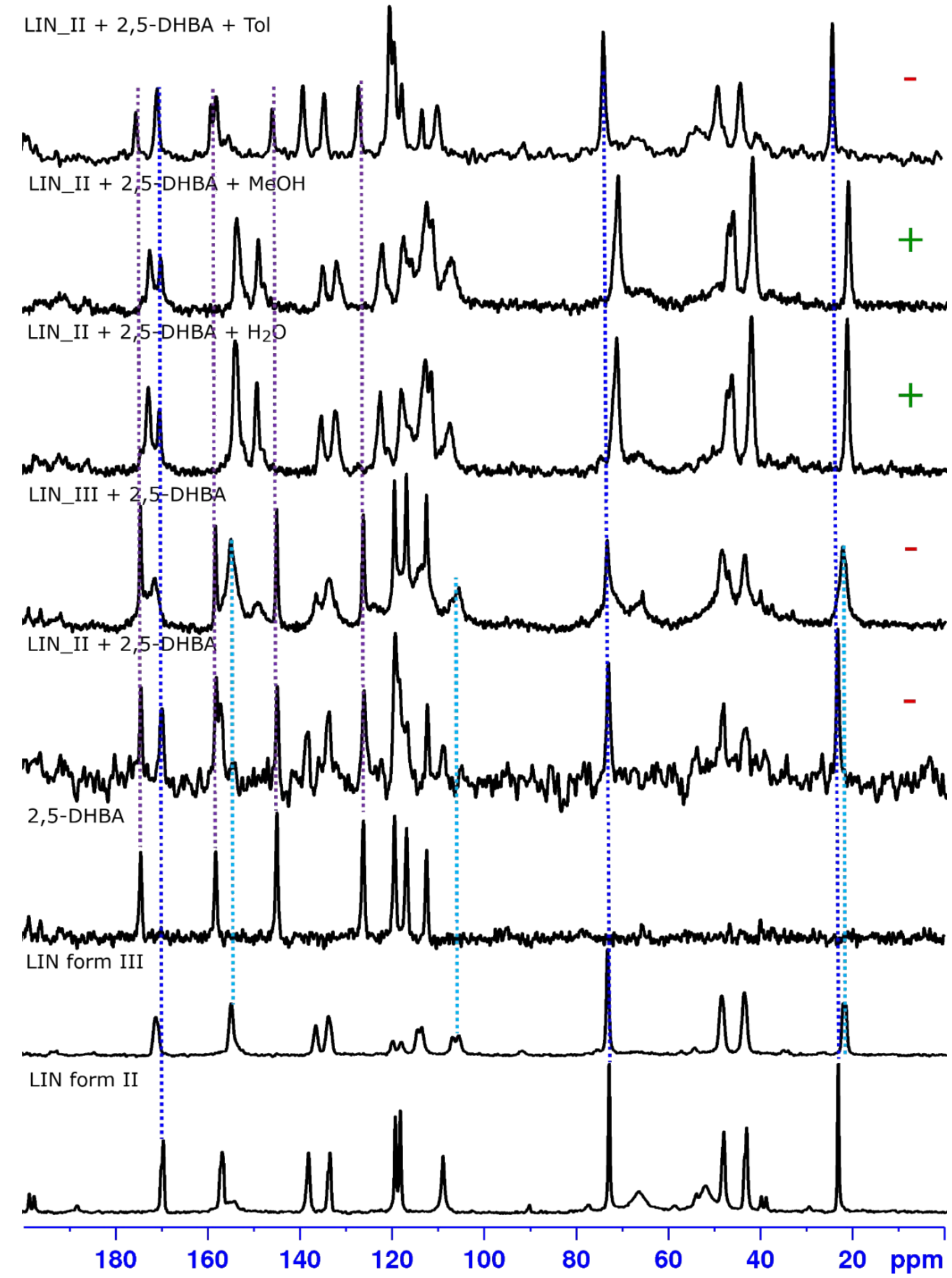

Figure S20. ${ }^{13} \mathrm{C}$ CPMAS NMR spectra for LIN form II, LIN form III, 2,5-DHBA and various reaction mixtures obtained after grinding LIN_II or LIN_III with 2,5-DHBA without any solvent and in the presence of water, methanol or toluene to create LAG conditions. Dashed lines indicate the positions of ${ }^{13} \mathrm{C}$ signals originating from pure reagents, 'plus' or 'minus' signs indicate whether a cocrystal was formed in given reaction conditions. 


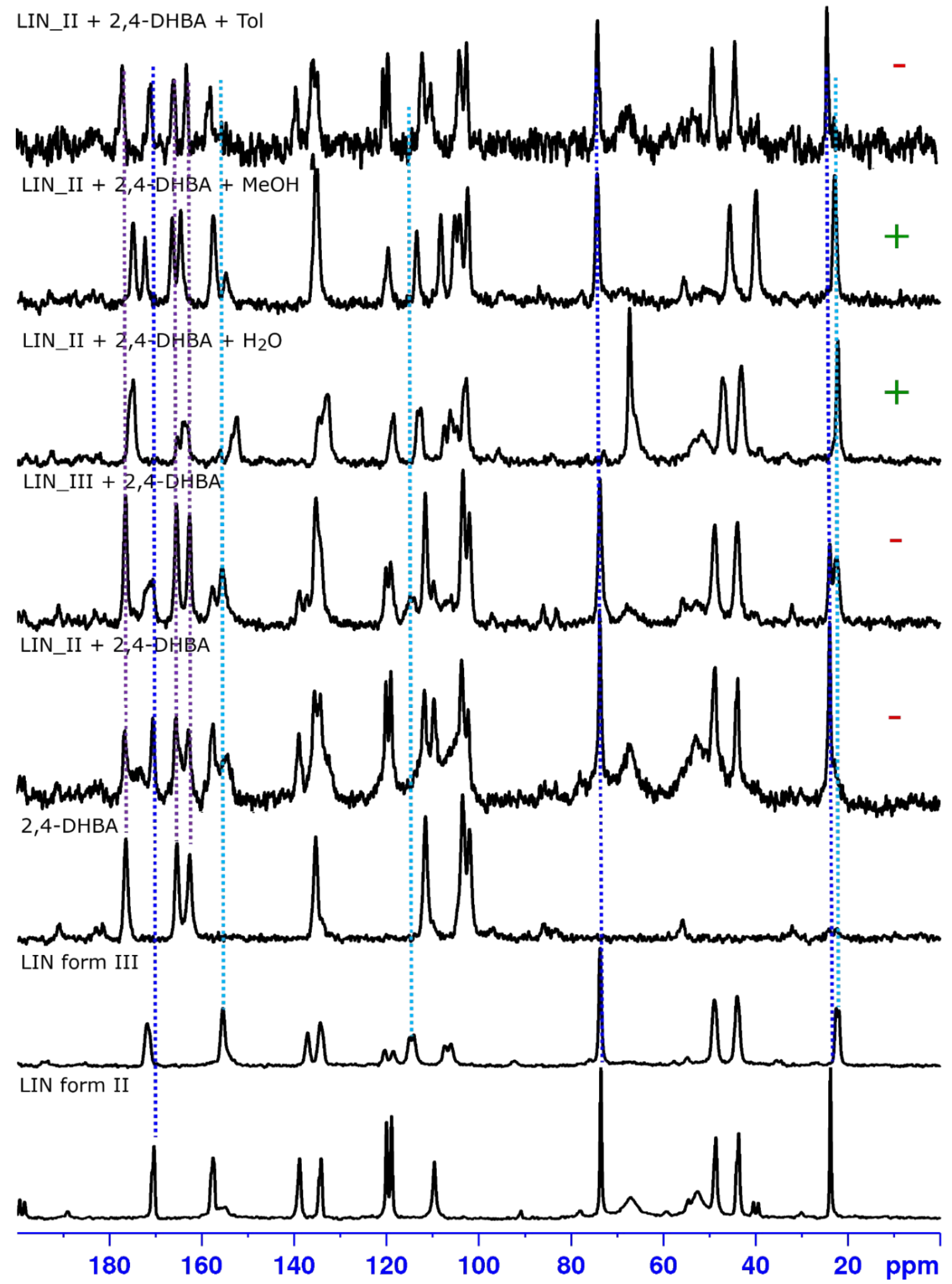

Figure S21. ${ }^{13} \mathrm{C}$ CPMAS NMR spectra for LIN form II, LIN form III, 2,4-DHBA and various reaction mixtures obtained after grinding LIN_II or LIN_III with 2,4-DHBA without any solvent and in the presence of water, methanol or toluene to create LAG conditions. Dashed lines indicate the positions of ${ }^{13} \mathrm{C}$ signals originating from pure reagents, 'plus' or 'minus' signs indicate whether a cocrystal was formed in given reaction conditions. 


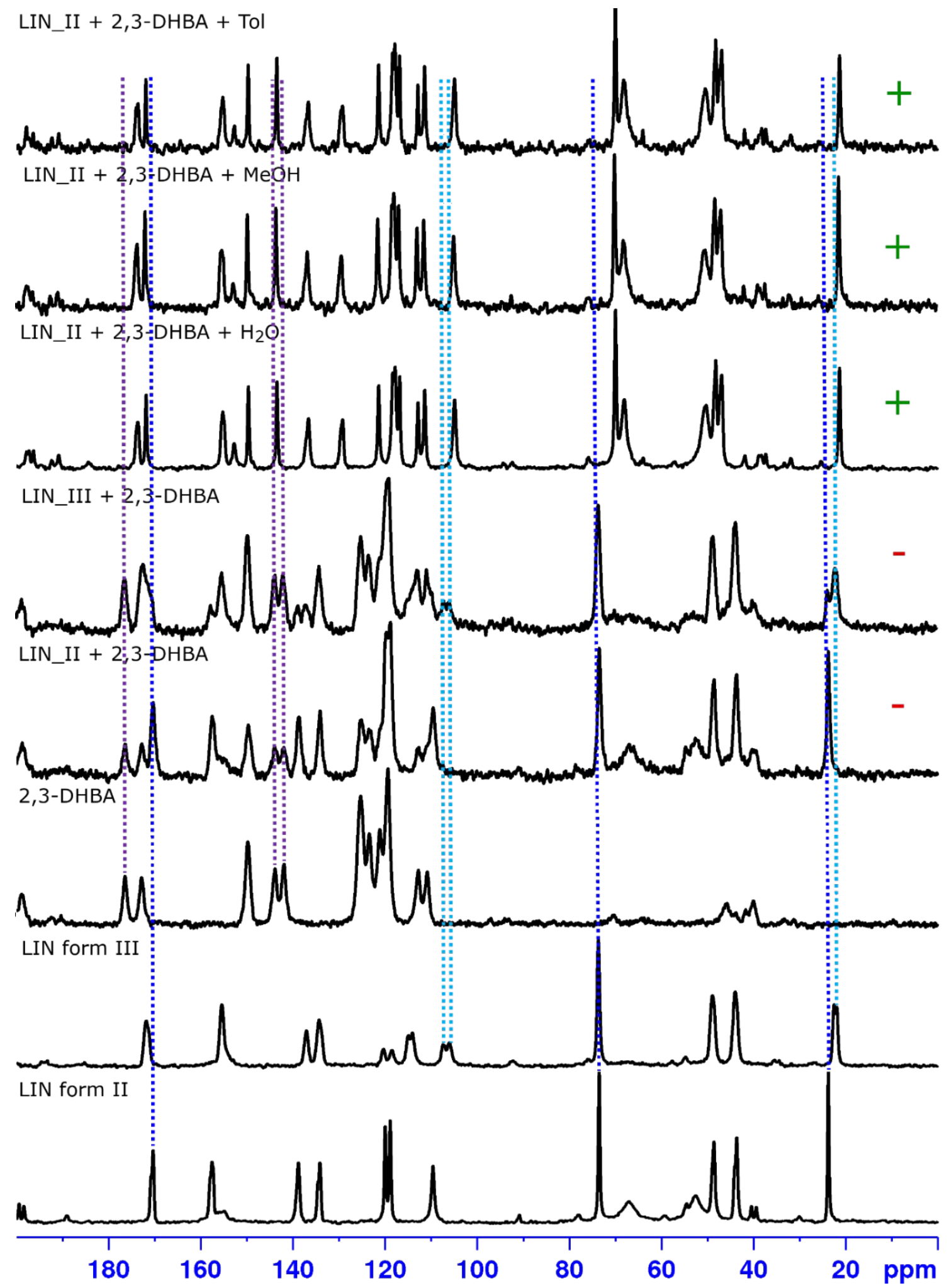

Figure S22. ${ }^{13} \mathrm{C}$ CPMAS NMR spectra for LIN form II, LIN form III, 2,3-DHBA and various reaction mixtures obtained after grinding LIN_II or LIN_III with 2,3-DHBA without any solvent and in the presence of water, methanol or toluene to create LAG conditions. Dashed lines indicate the positions of ${ }^{13} \mathrm{C}$ signals originating from pure reagents, 'plus' or 'minus' signs indicate whether a cocrystal was formed in given reaction conditions. 


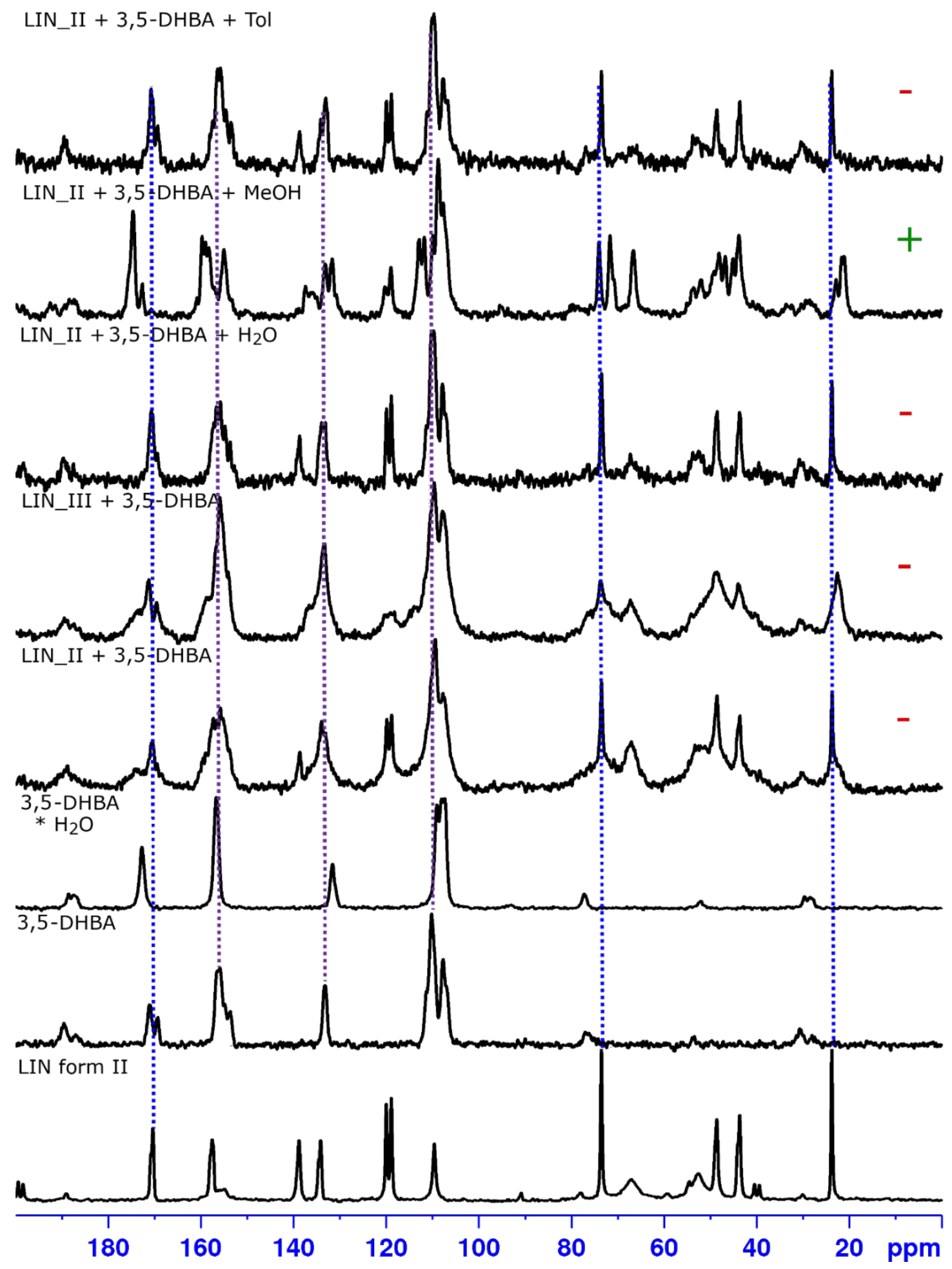

Figure S23. ${ }^{13} \mathrm{C}$ CPMAS NMR spectra for LIN form II, 3,5-DHBA, 3,5-DHBA $* \mathrm{H}_{2} \mathrm{O}$ and various reaction mixtures obtained after grinding LIN_II or LIN_III with 3,5-DHBA without any solvent and in the presence of water, methanol or toluene to create LAG conditions. Dashed lines indicate the positions of ${ }^{13} \mathrm{C}$ signals originating from pure reagents, 'plus' or 'minus' signs indicate whether a cocrystal was formed in given reaction conditions. 


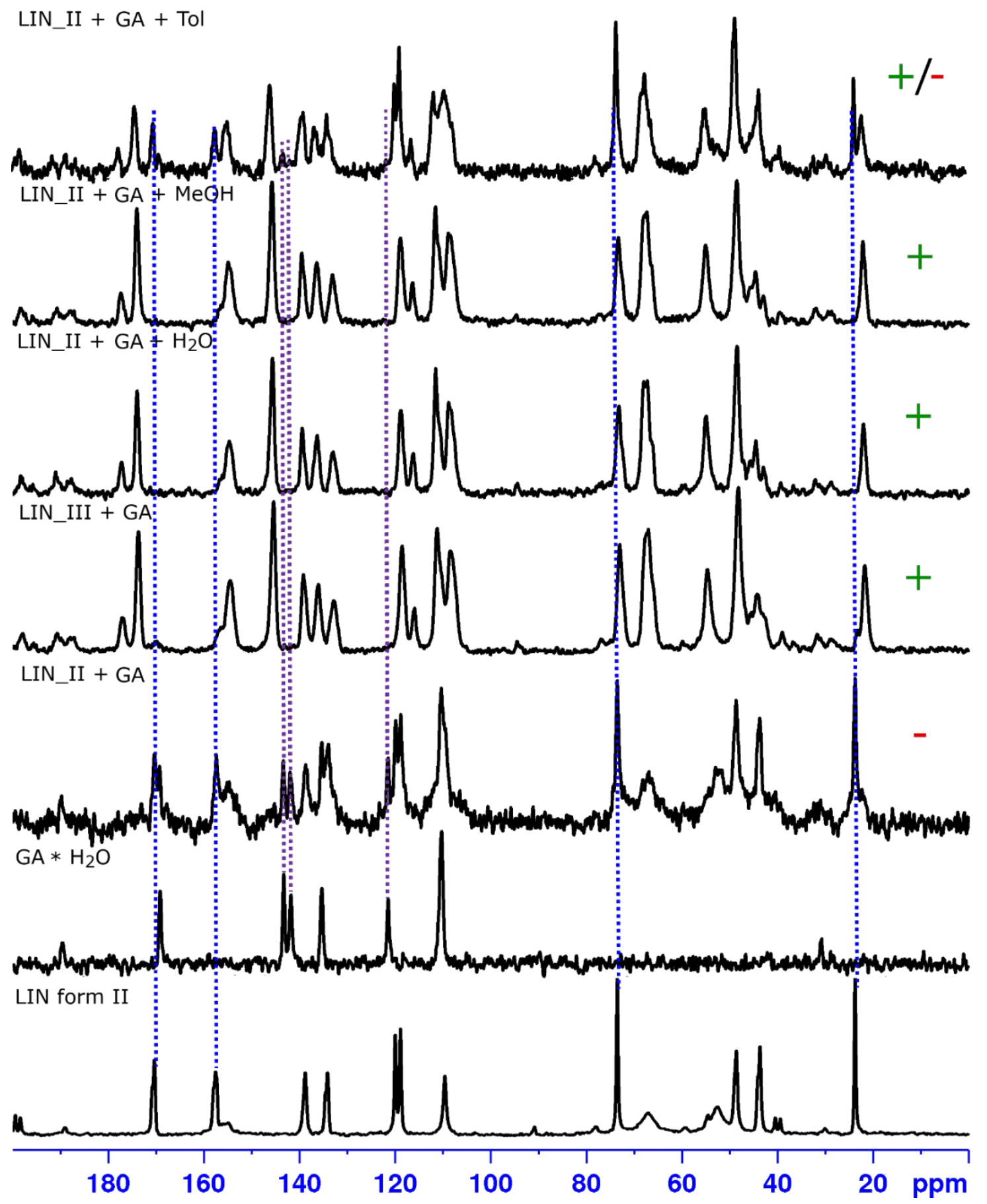

Figure S24. ${ }^{13} \mathrm{C}$ CPMAS NMR spectra for LIN form II, GA* $\mathrm{H}_{2} \mathrm{O}$ and various reaction mixtures obtained after grinding LIN_II or LIN_III with GA without any solvent and in the presence of water, methanol or toluene to create LAG conditions. Dashed lines indicate the positions of ${ }^{13} \mathrm{C}$ signals originating from pure reagents, 'plus' or 'minus' signs indicate whether a cocrystal was formed in given reaction conditions. 


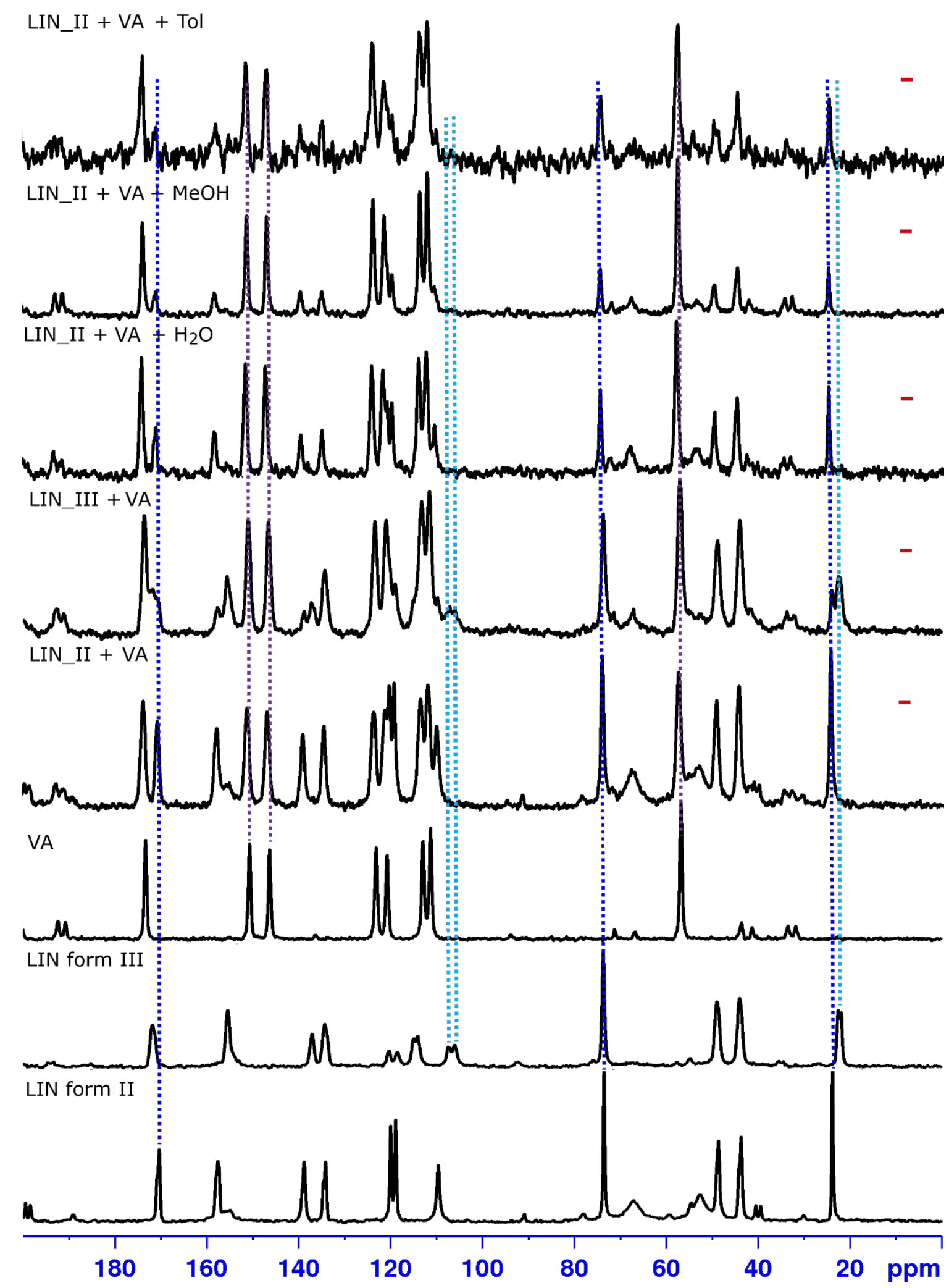

Figure S25. ${ }^{13} \mathrm{C}$ CPMAS NMR spectra for LIN form II, LIN form III, VA and various reaction mixtures obtained after grinding LIN_II or LIN_III with VA without any solvent and in the presence of water, methanol or toluene to create LAG conditions. Dashed lines indicate the positions of ${ }^{13} \mathrm{C}$ signals originating from pure reagents, 'minus' sign indicates a lack of cocrystal formation in given reaction conditions. 


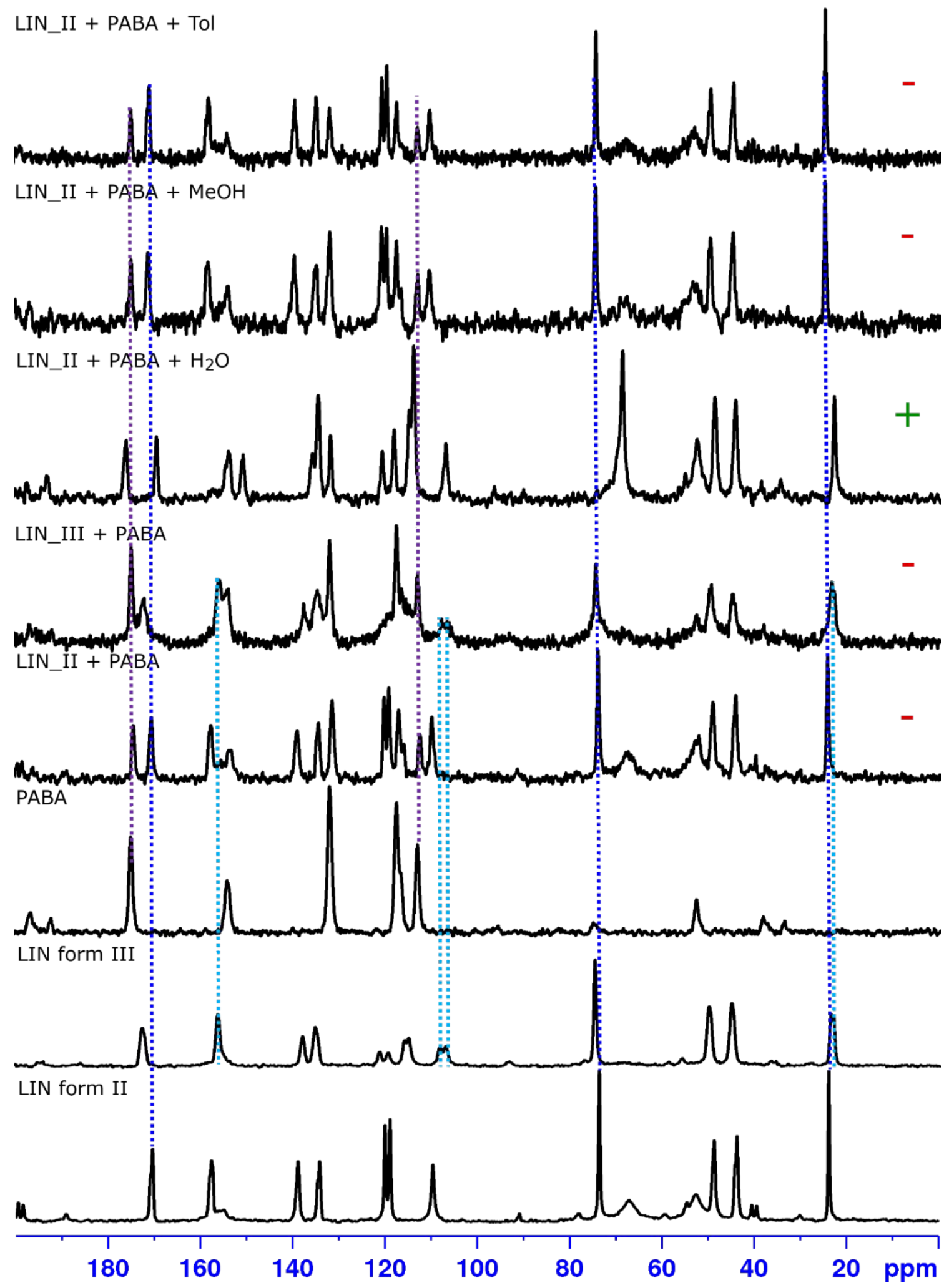

Figure S26. ${ }^{13} \mathrm{C}$ CPMAS NMR spectra for LIN form II, LIN form III, PABA and various reaction mixtures obtained after grinding LIN_II or LIN_III with PABA without any solvent and in the presence of water, methanol or toluene to create LAG conditions. Dashed lines indicate the positions of ${ }^{13} \mathrm{C}$ signals originating from pure reagents, 'plus' or 'minus' signs indicate whether a cocrystal was formed in given reaction conditions. 


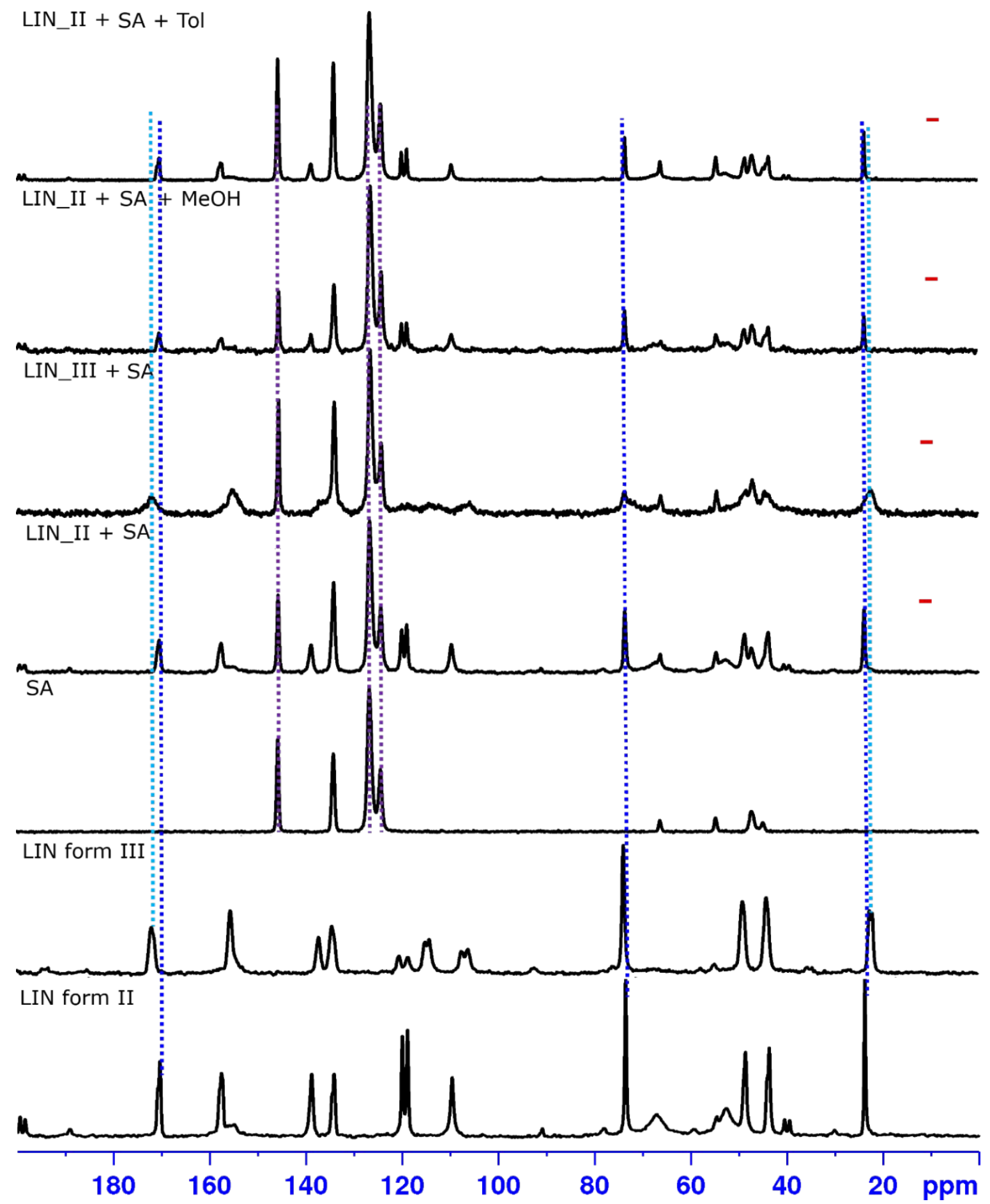

Figure S27. ${ }^{13} \mathrm{C}$ CPMAS NMR spectra for LIN form II, LIN form III, SA and various reaction mixtures obtained after grinding LIN_II or LIN_III with SA without any solvent and in the presence of water, methanol or toluene to create LAG conditions. Dashed lines indicate the positions of ${ }^{13} \mathrm{C}$ signals originating from pure reagents, 'minus' sign indicates a lack of cocrystal formation in given reaction conditions. 


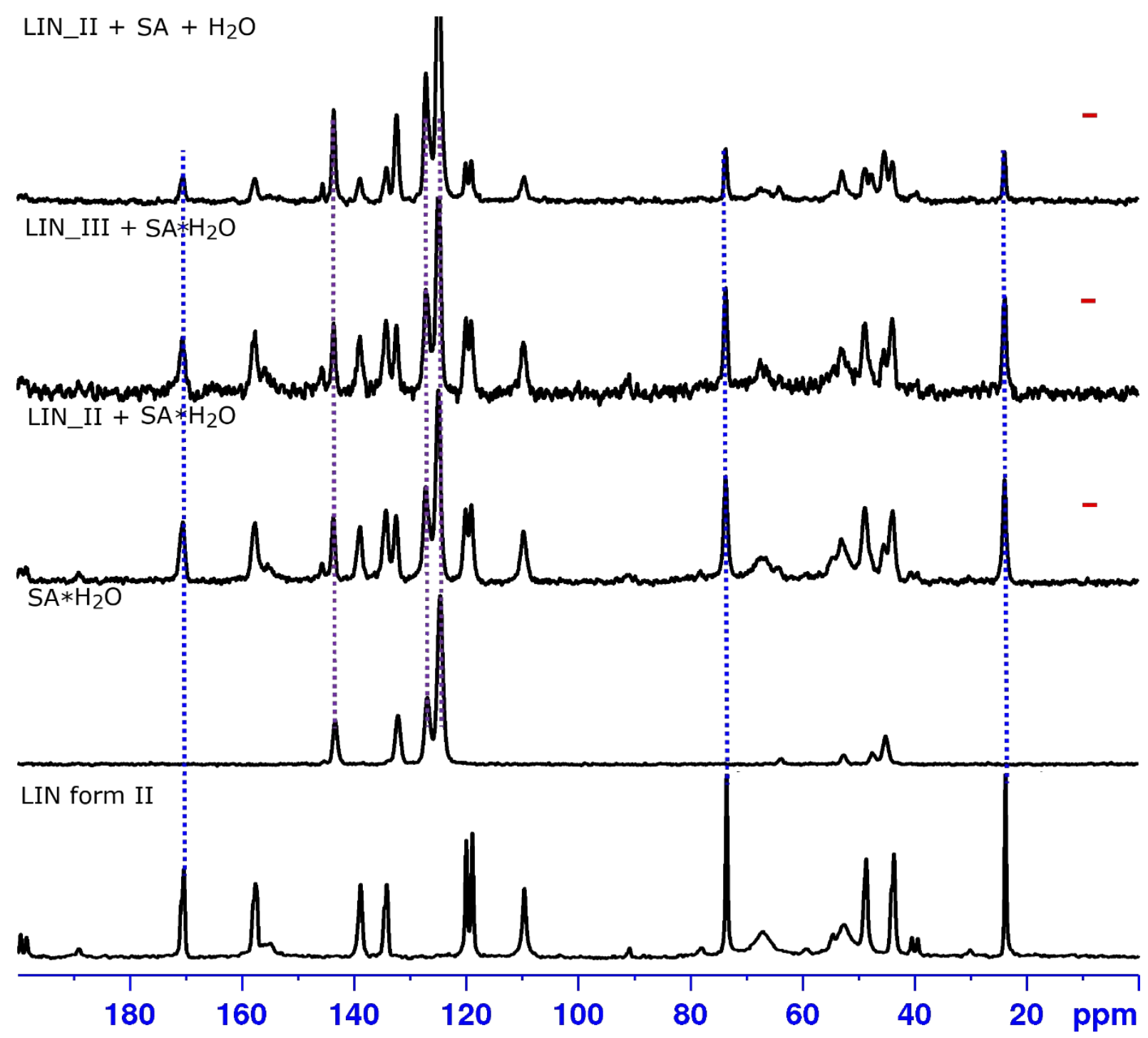

Figure S28. ${ }^{13} \mathrm{C}$ CPMAS NMR spectra for $\mathrm{LIN}$ form II, SA* $\mathrm{H}_{2} \mathrm{O}$ and various reaction mixtures obtained after grinding LIN_II or LIN_III with SA* $\mathrm{H}_{2} \mathrm{O}$ without any solvent and in the presence of water, methanol or toluene to create LAG conditions. Dashed lines indicate the positions of ${ }^{13} \mathrm{C}$ signals originating from pure reagents, 'minus' sign indicates a lack of cocrystal formation in given reaction conditions. 


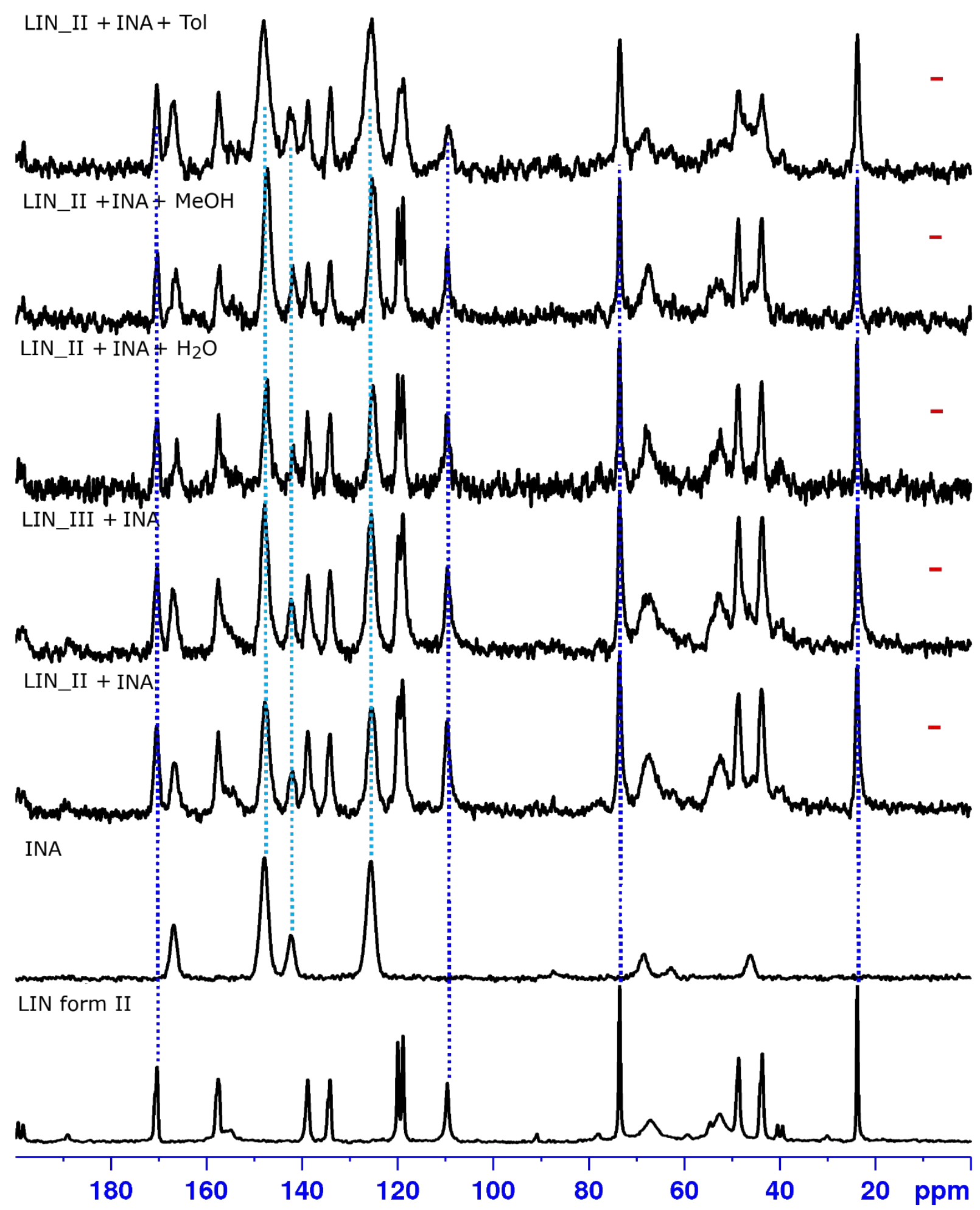

Figure S29. ${ }^{13} \mathrm{C}$ CPMAS NMR spectra for LIN form II, INA and various reaction mixtures obtained after grinding LIN_II or LIN_III with INA without any solvent and in the presence of water, methanol or toluene to create $\mathrm{LAG}$ conditions. Dashed lines indicate the positions of ${ }^{13} \mathrm{C}$ signals originating from pure reagents, 'minus' sign indicates a lack of cocrystal formation in given reaction conditions. 


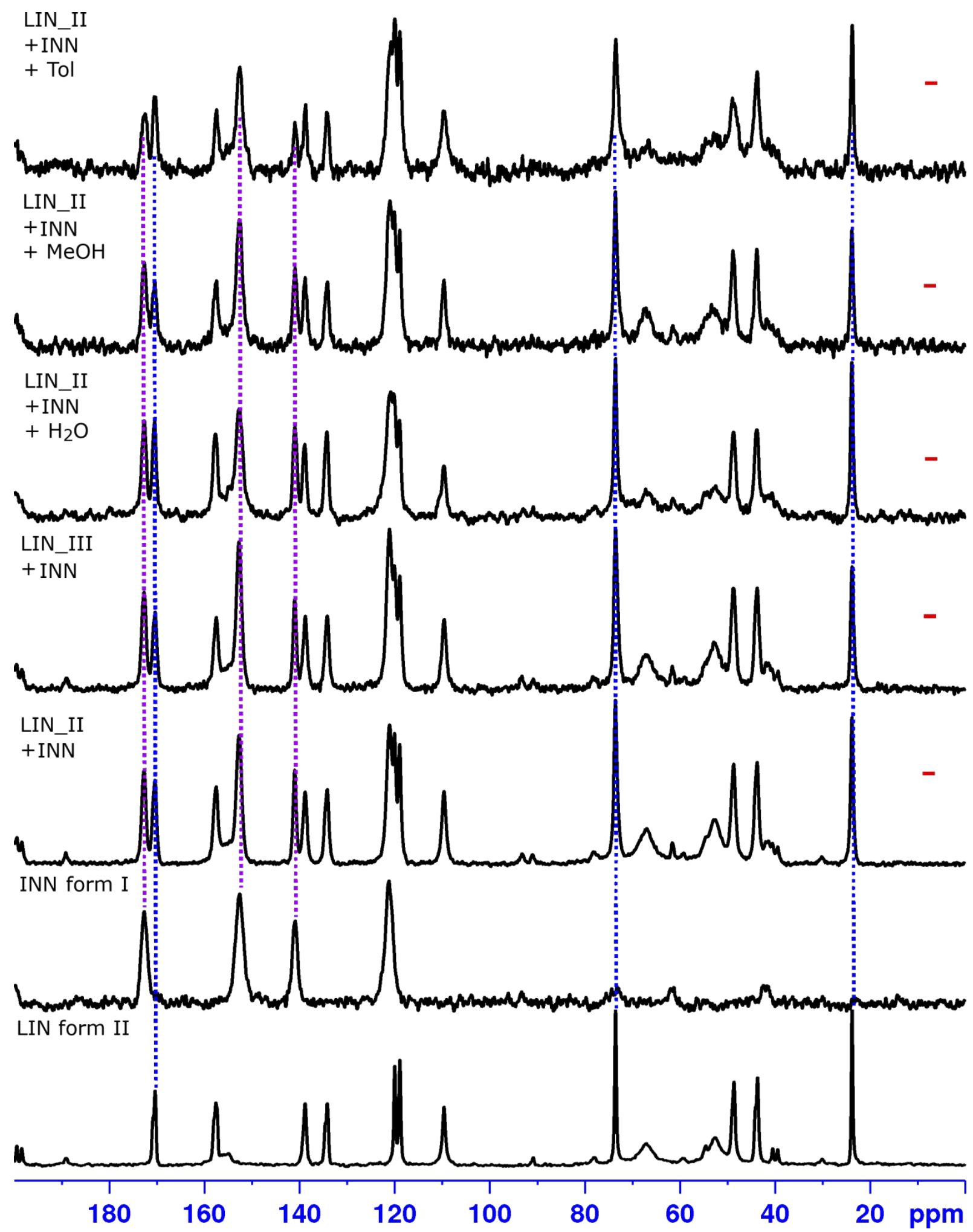

Figure S30. ${ }^{13} \mathrm{C}$ CPMAS NMR spectra for LIN form II, INN form I and various reaction mixtures obtained after grinding LIN_II or LIN_III with INN without any solvent and in the presence of water, methanol or toluene to create LAG conditions. Dashed lines indicate the positions of ${ }^{13} \mathrm{C}$ signals originating from pure reagents, 'minus' sign indicates a lack of cocrystal formation in given reaction conditions. 


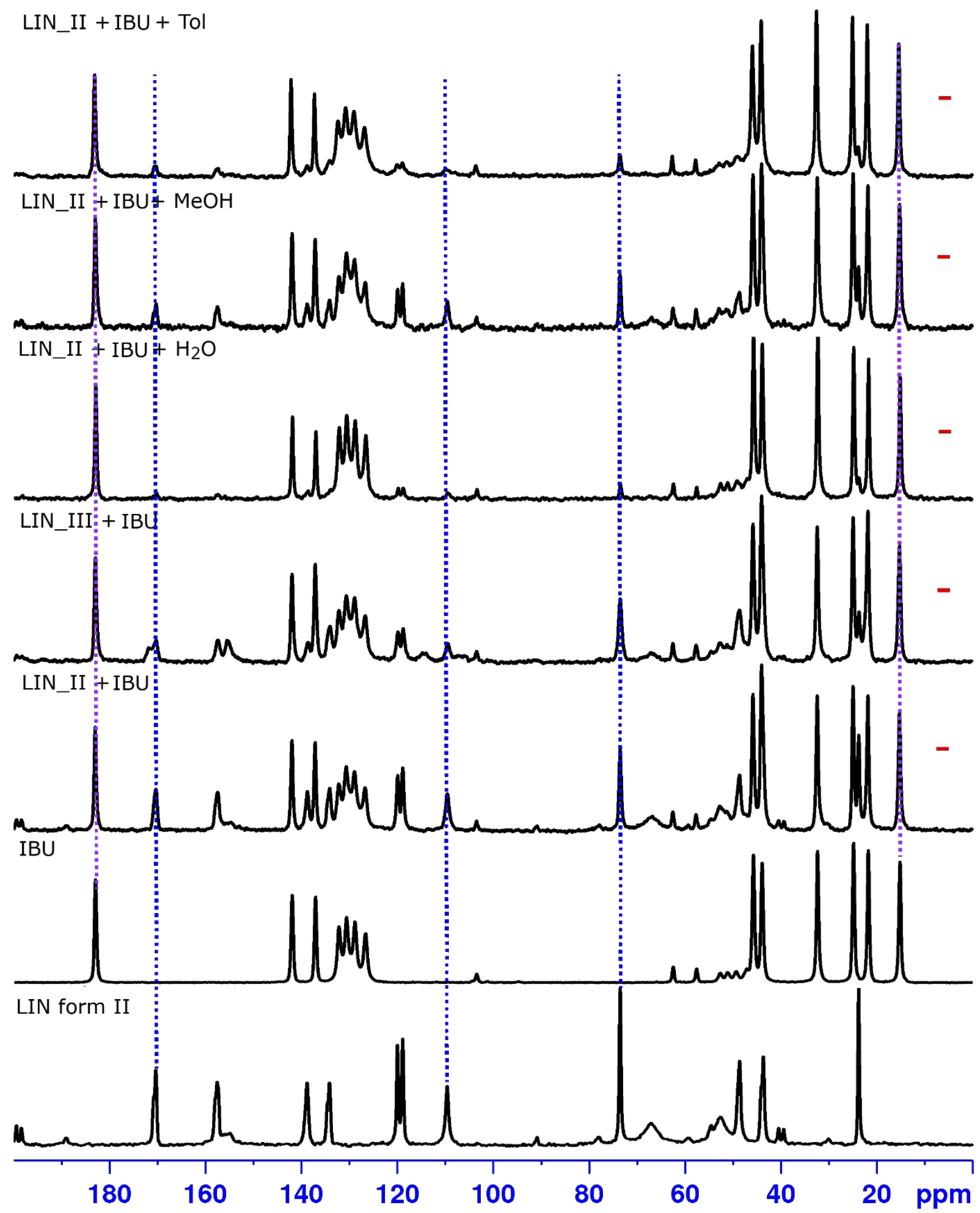

Figure S31. ${ }^{13} \mathrm{C}$ CPMAS NMR spectra for LIN form II, IBU and various reaction mixtures obtained after grinding LIN_II or LIN_III with VA without any solvent and in the presence of water, methanol or toluene to create LAG conditions. Dashed lines indicate the positions of ${ }^{13} \mathrm{C}$ signals originating from pure reagents, 'minus' sign indicates a lack of cocrystal formation in given reaction conditions. 


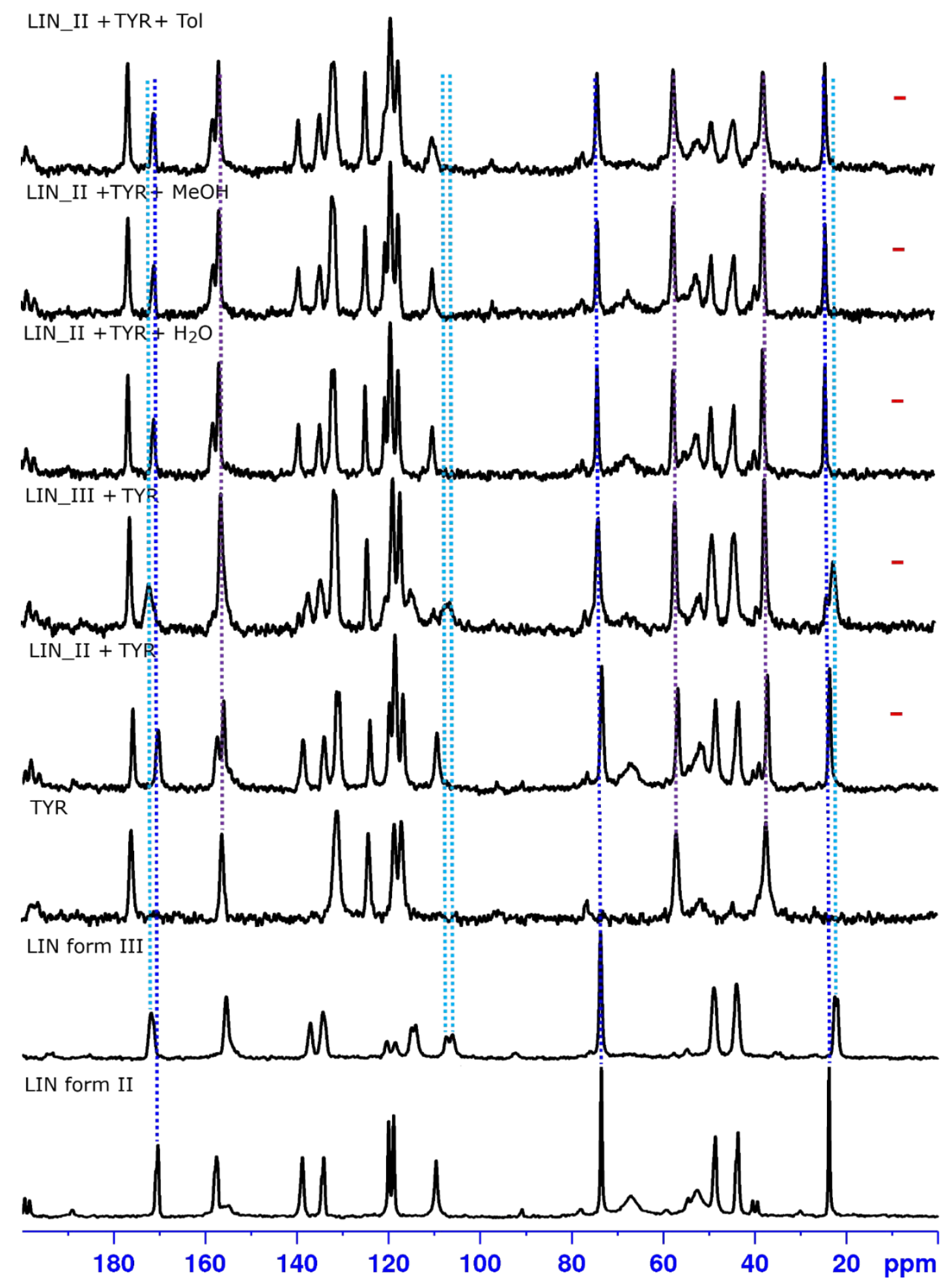

Figure S32. ${ }^{13} \mathrm{C}$ CPMAS NMR spectra for LIN form II, LIN form III, TYR and various reaction mixtures obtained after grinding LIN_II or LIN_III with TYR without any solvent and in the presence of water, methanol or toluene to create LAG conditions. Dashed lines indicate the positions of ${ }^{13} \mathrm{C}$ signals originating from pure reagents, 'minus' sign indicates a lack of cocrystal formation in given reaction conditions. 


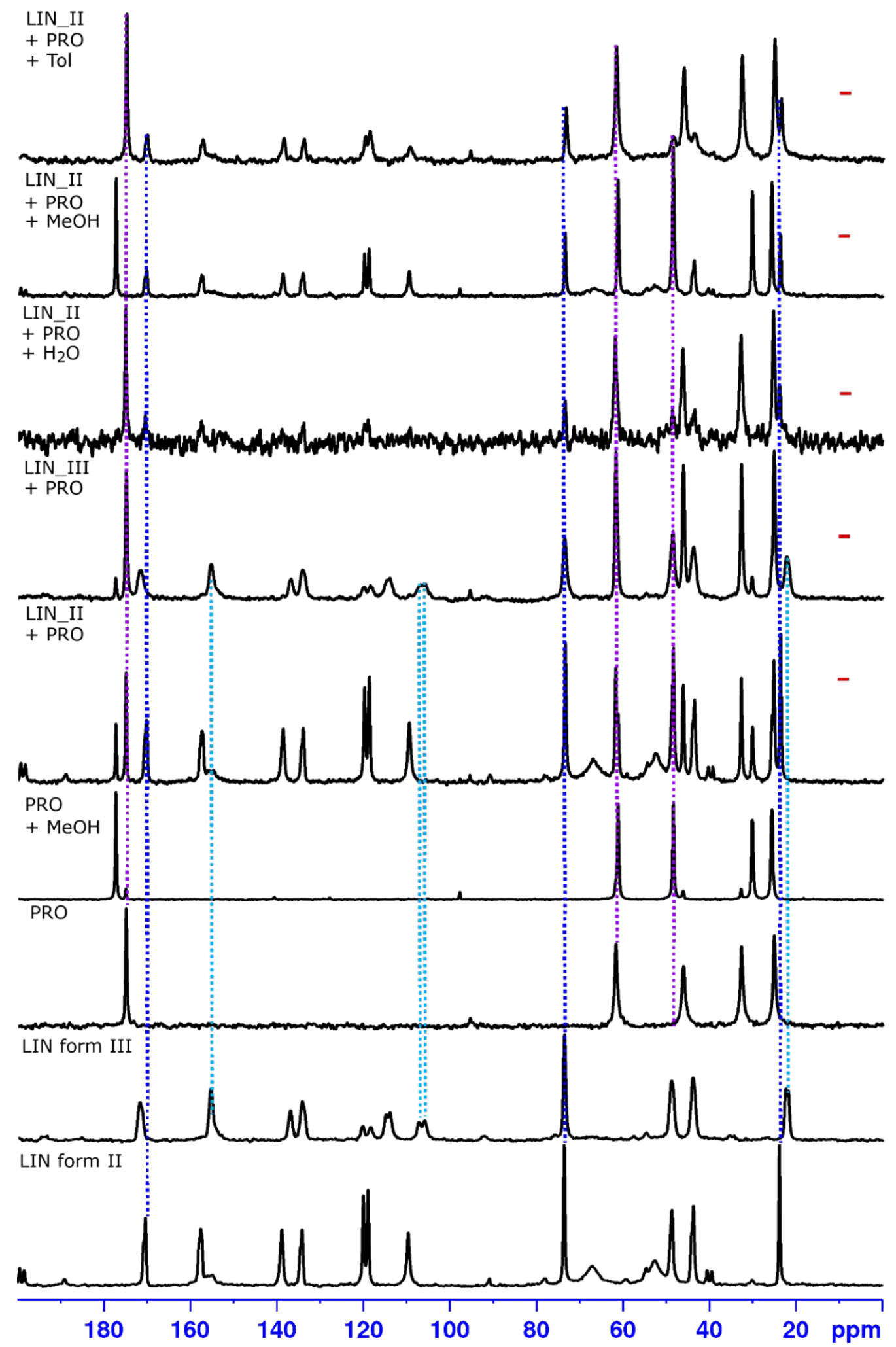

Figure S33. ${ }^{13} \mathrm{C}$ CPMAS NMR spectra for LIN form II, LIN form III, PRO and various reaction mixtures obtained after grinding LIN_II or LIN_III with PRO without any solvent and in the presence of water, methanol or toluene to create LAG conditions, as well as after grinding PRO with methanol. Dashed lines indicate the positions of ${ }^{13} \mathrm{C}$ signals originating from pure reagents, 'minus' sign indicates a lack of cocrystal formation in given reaction conditions. 


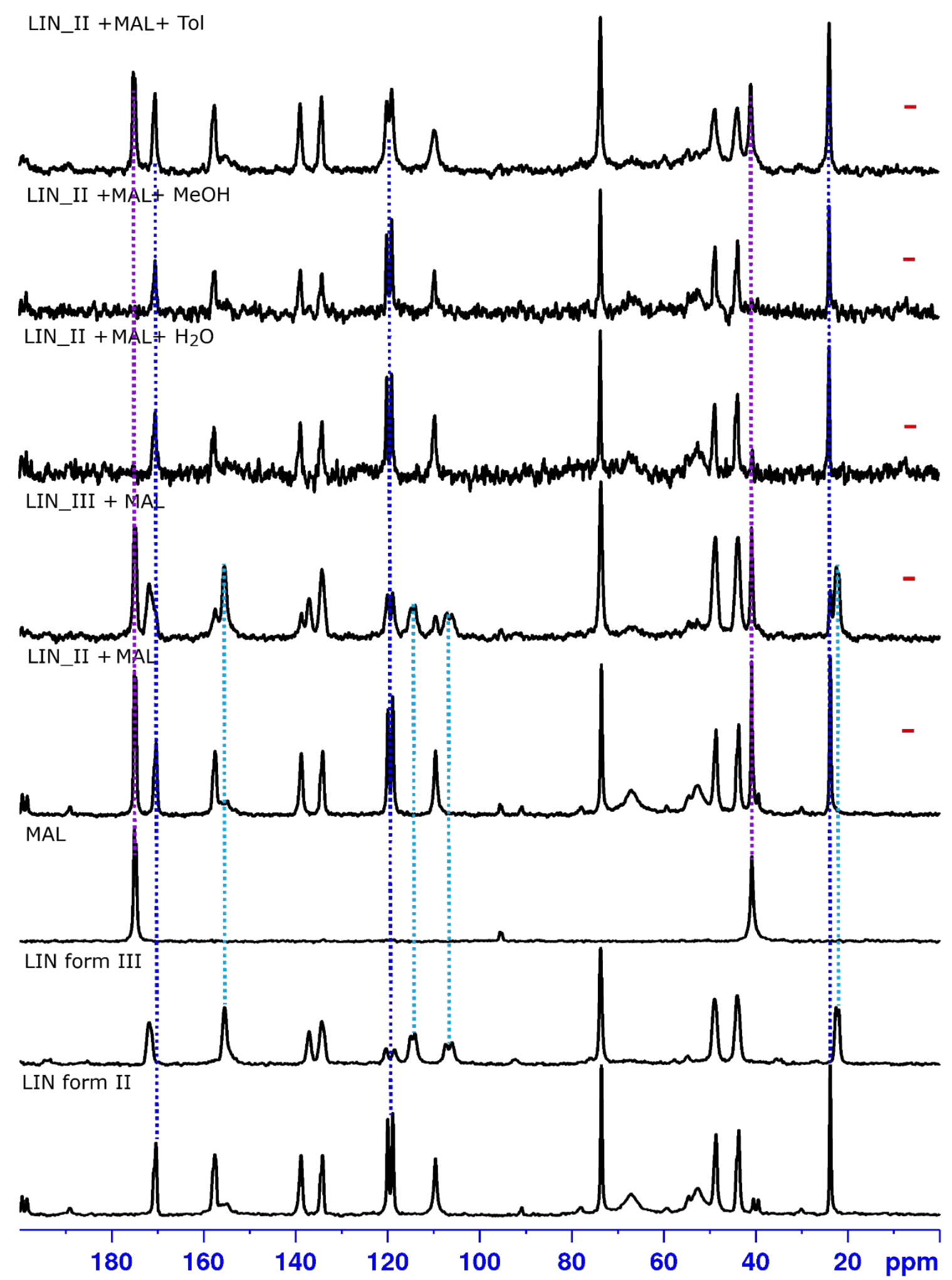

Figure S34. ${ }^{13} \mathrm{C}$ CPMAS NMR spectra for LIN form II, LIN form III, MAL and various reaction mixtures obtained after grinding LIN_II or LIN_III with MAL without any solvent and in the presence of water, methanol or toluene to create LAG conditions. Dashed lines indicate the positions of ${ }^{13} \mathrm{C}$ signals originating from pure reagents, 'minus' sign indicates a lack of cocrystal formation in given reaction conditions. 


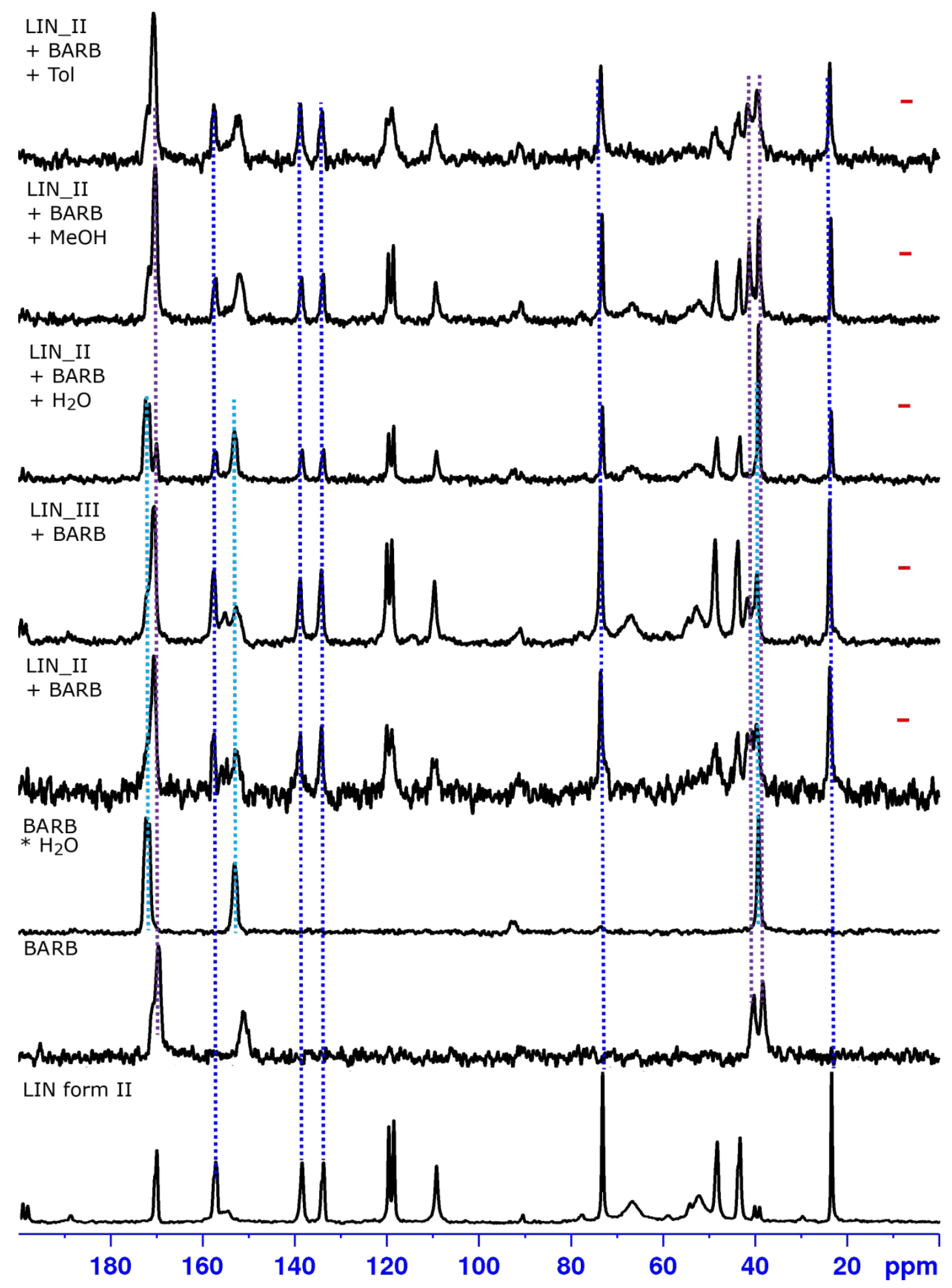

Figure S35. ${ }^{13} \mathrm{C}$ CPMAS NMR spectra for LIN form II, BARB, BARB* $\mathrm{H}_{2} \mathrm{O}$ and various reaction mixtures obtained after grinding LIN_II or LIN_III with BARB without any solvent and in the presence of water, methanol or toluene to create LAG conditions. Dashed lines indicate the positions of ${ }^{13} \mathrm{C}$ signals originating from pure reagents, 'minus' sign indicates a lack of cocrystal formation in given reaction conditions. 


\section{Determination of the coformers polymorphic forms}

Benzoic acid (BA) crystallizes in one polymorphic P2 $2_{1} / \mathrm{c}$ monoclinic form (CSD refcode $\mathrm{BENZAC).} \mathrm{For} \mathrm{all} \mathrm{calculations} \mathrm{BENZAC1} 3^{1}$ crystal structure from the CSD database was used.

2,3-dihydroxybenzoic acid (2,3-DHBA) has two known polymorphic forms: triclinic P-1 $(\mathrm{CSD} \text { refcode CACDAM })^{2}$ and monoclinic $\mathrm{P} 2{ }_{1} / \mathrm{n}\left(\mathrm{CSD}\right.$ refcode CACDAM01). ${ }^{3}$ Of these, only the triclinic form is a $Z^{\prime}=2$ structure, in agreement with the ${ }^{13} \mathrm{C}$ CPMAS NMR spectrum registered for our sample of this coformer (see Figure S36). Therefore the crystal structure of this triclinic form was accounted for in the calculations.

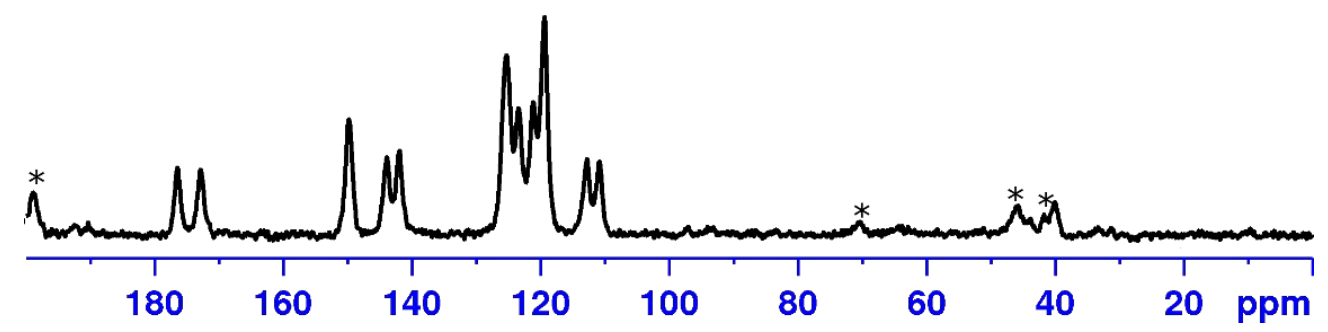

Figure S36. ${ }^{13} \mathrm{C}$ CPMAS NMR spectrum of commercial 2,3-DHBA sample. The number of ${ }^{13} \mathrm{C}$ resonances indicates a $Z^{\prime}=2$ structure, which corresponds to a triclinic form of 2,3-DHBA. The repetition delay for this spectrum was equal to $300 \mathrm{~s}$. Asterisks mark spinning sidebands.

2,4-dihydroxybenzoic acid (2,4-DHBA) exists in two monoclinic forms, form I (CSD refcode ZZZEEU08) ${ }^{4}$ and form II (CSD refcode ZZZEEU01). ${ }^{5}$ The forms are enantiotropically related, with form II being more stable at room temperature and showing in the DSC a phase transition to form I at ca. $155-160{ }^{\circ} \mathrm{C} .{ }^{6}$ The DSC plot registered for the commercial sample of 2,4-DHBA shows an endothermic transition corresponding to this phase change (Figure S37). Therefore it was form II of 2,4-DHBA that was taking part for mechanochemical reactions.

2,4-DHBA is also known to form two hydrates: a hemihydrates (CSD refcode QIVTUK01)4 and a monohydrate (CSD refcode YUXGUV). ${ }^{7}$ In our conditions neither of these forms were observed. Even after grinding 2,4-DHBA with water or methanol no hydrate formation was observed, as indicated by a lack of any change in the ${ }^{13} \mathrm{C}$ CPMAS NMR spectra of 2,4-DHBA before and after grinding with water or methanol (see Figure S37). 


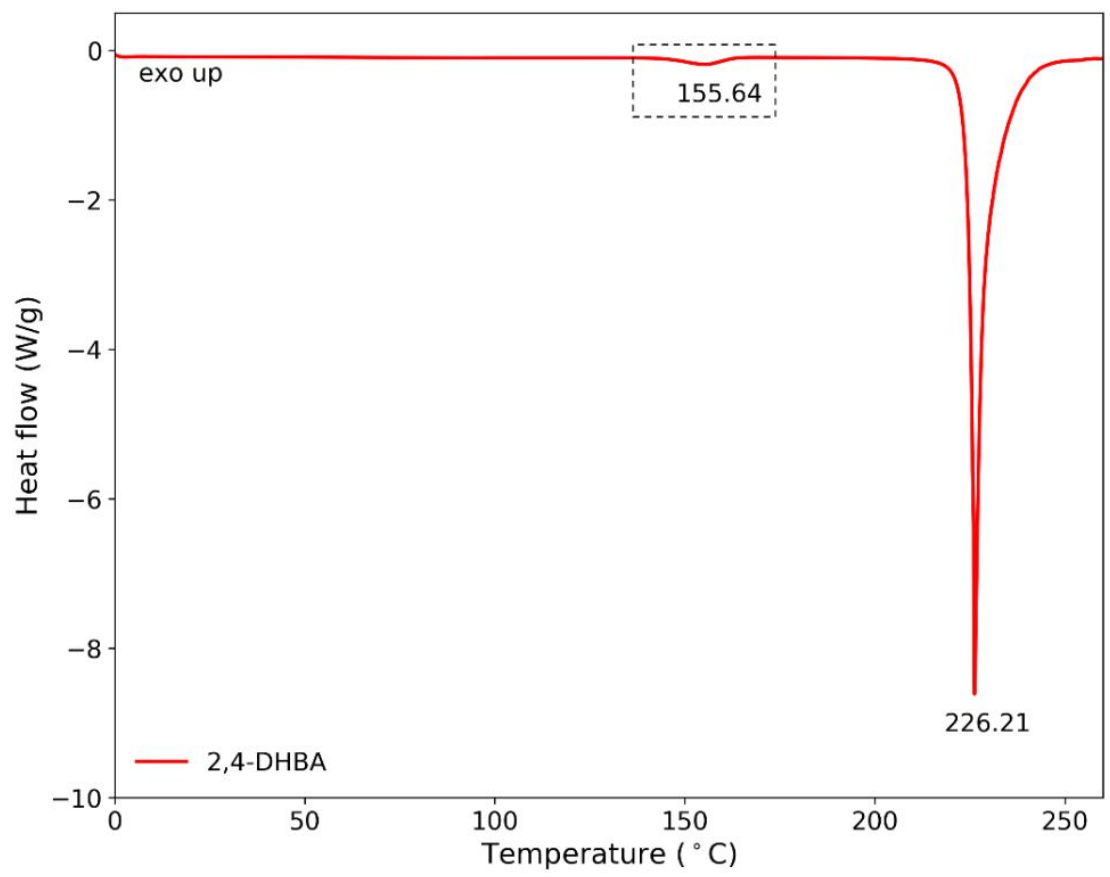

2,4-DHBA ground with $\mathrm{MeOH}$

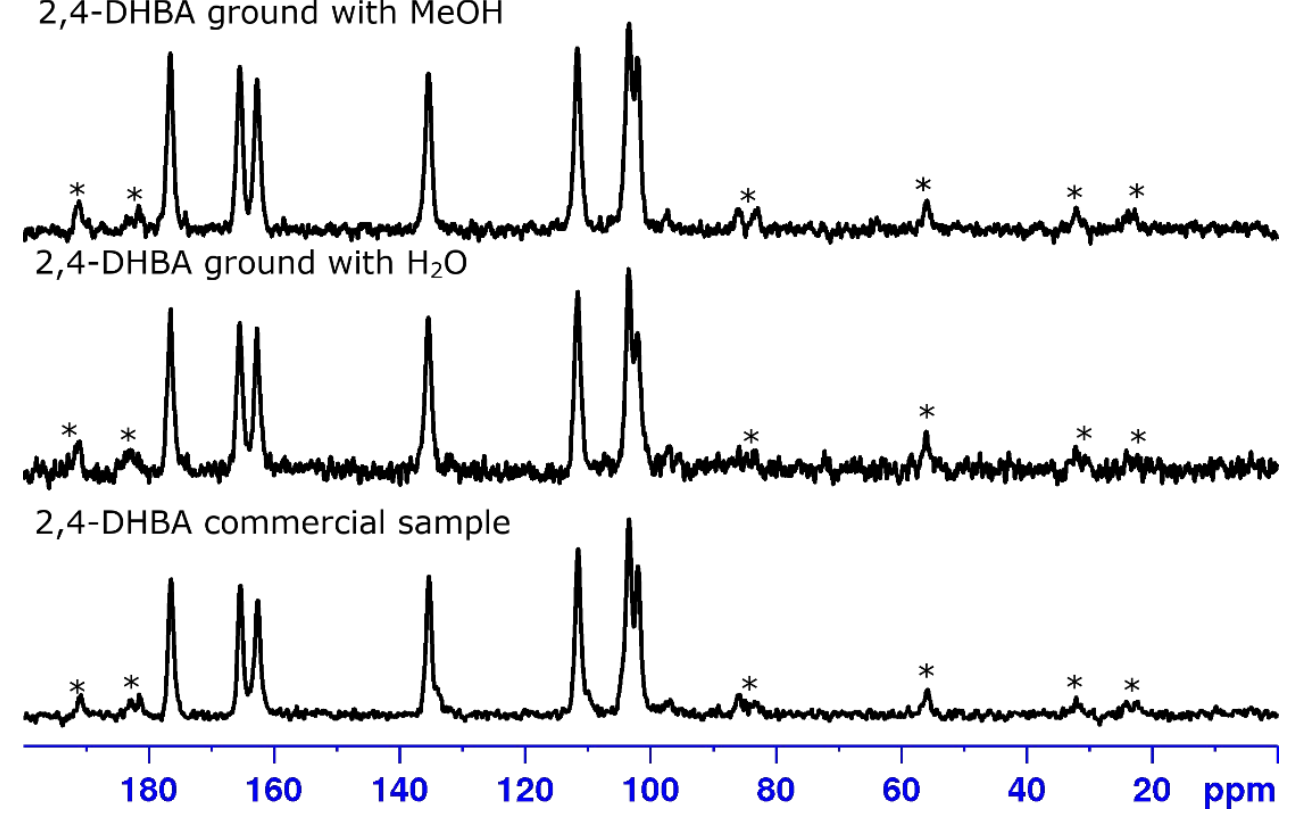

Figure S37. Upper: the DSC curve for a commercial sample of 2,4-DHBA with a phase transition from form II to form I marked by a dashed-lined rectangle; lower: ${ }^{13} \mathrm{C}$ CPMAS NMR spectra registered for a commercial sample of 2,4-DHBA and the same sample after grinding with water or methanol. Asterisks mark spinning sidebands.

2,5-dihydroxybenzoic acid (2,5-DHBA) has also two known polymorphic forms (both monoclinic): form I (CSD refcode BESKAL02) ${ }^{8}$ and form II (CSD refcode BESKAL03). 8 The ${ }^{13} \mathrm{C}$ CPMAS NMR spectrum registered for the commercial sample of 2,5-DHBA indicated that it is a mixture of two polymorphic forms, which was deduced from the presence of additional small intensity signals apart from those detected for the major form present in the sample. The DSC measurements confirmed the presence of two polymorphs, as indicated by two separate melting events at temperatures corresponding to the melting points of form I $\left(207.7^{\circ} \mathrm{C}\right)$ and II 
$\left(203.6^{\circ} \mathrm{C}\right)$, respectively.6 After recrystallization of this sample from 2-propanol/methanol only one set of signals was visible in the ${ }^{13} \mathrm{C}$ CPMAS spectrum of this sample and only one endothermic event on the DSAC curve corresponding to the melting point of form I (see Figure S38). In the grinding experiments with LIN form I of 2,5-DHBA was used.
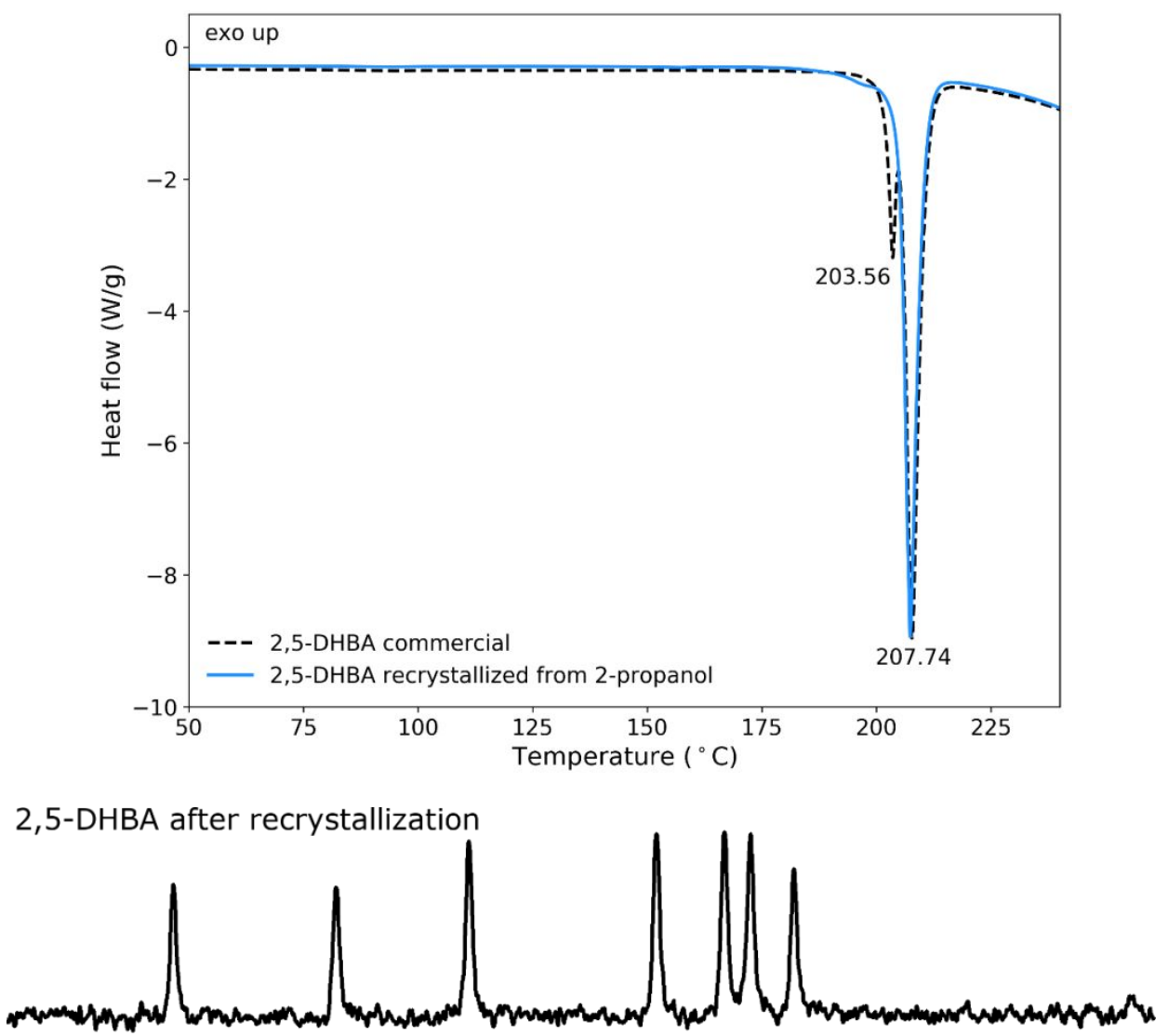

2,5-DHBA commercial sample

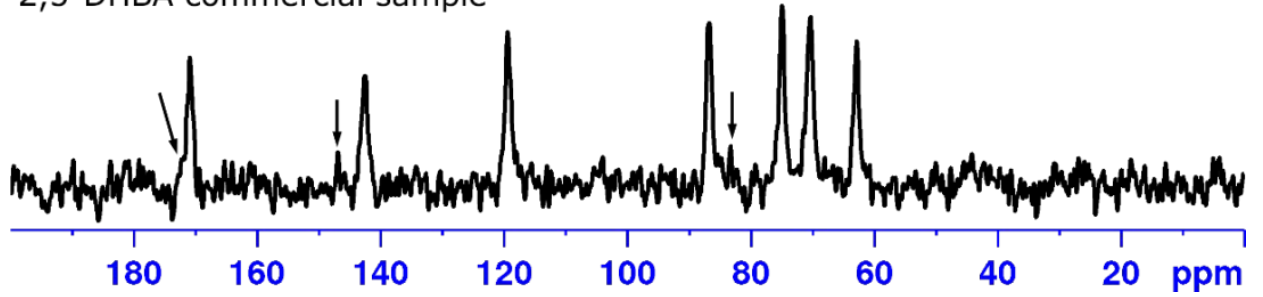

Figure S38. The DSC curves (upper) and ${ }^{13} \mathrm{C}$ CPMAS NMR spectra (lower) registered for a commercial sample of 2,5-DHBA and the same sample after recrystallization from 2propanol/methanol. Arrows in the NMR spectrum of commercial 2,5-DHBA mark additional signals originating from form II.

2,6-dihydroxybenzoic acid (2,6-DHBA) crystallizes in two neat polymorphic forms: orthorhombic $P$ na $2_{1}$ form (CSD refcode LEZJAB) ${ }^{9}$ and monoclinic $P 2_{1} / \mathrm{c}$ form (CSD refcode LEZJAB01), ${ }^{10}$ in addition to a monohydrate Pnma form (CSD refcode LEZJEF). ${ }^{9}$ The ${ }^{13} \mathrm{C}$ CPMAS NMR spectrum registered for commercial sample of 2,6-DHBA indicates the presence of a mixture of polymorphic forms, as indicated by small intensity signals marked in Figure S39 by arrows. This is further corroborated by the PXRD measurements, which suggest that the commercial sample contains $P$ na $2_{1}$ form with an admixture of the monohydrate form (see Figure S39 for reflexes originating from both forms clearly identifiable in the PXRD 
diffractogram of 2,6-DHBA sample). The DSC curve for the commercial sample of 2,6-DHBA indicates the presence of the monoclinic form, with the melting point of ca. $164^{\circ} \mathrm{C}$, in agreement with the literature values $\left(165-167{ }^{\circ} \mathrm{C}\right),{ }^{11}$ with a small endothermic event at ca. $95{ }^{\circ} \mathrm{C}$ in the DSC associated with a loss of water from a monohydrate form.
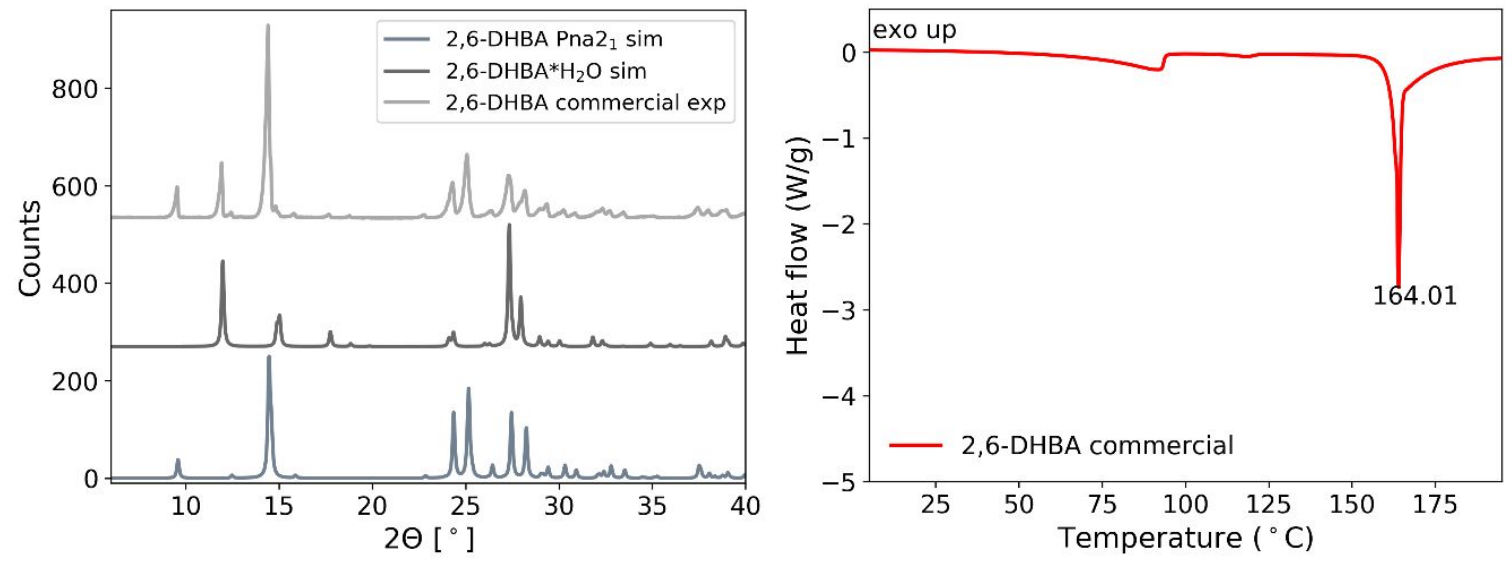

2,6-DHBA ground with $\mathrm{MeOH}$

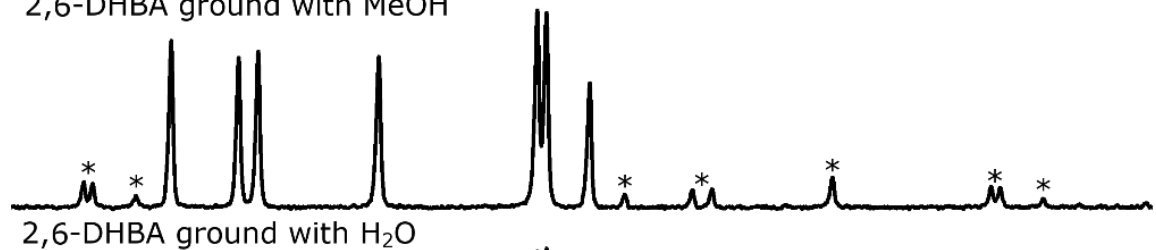

2,6-DHBA ground with $\mathrm{H}_{2} \mathrm{O}$

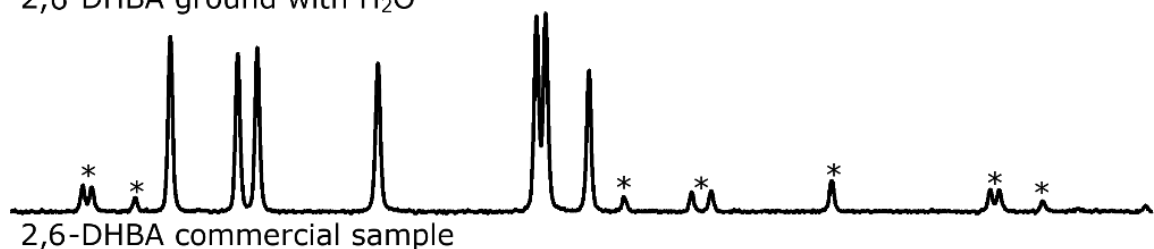

2,6-DHBA commercial sample

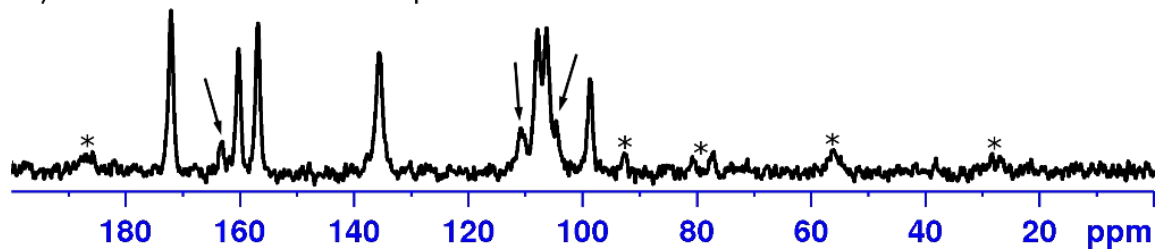

Figure S39. Upper left: experimental PXRD data for the commercial sample of 2,6-DHBA and simulated PXRD patterns for its orthorhombic form (LEZJAB), as well as its monohydrate (LEZJEF); upper right: the DSC curve for a commercial sample of 2,6-DHBA; lower: ${ }^{13} \mathrm{C}$ CPMAS NMR spectra registered for a commercial sample of 2,6-DHBA and the same sample after grinding with water or methanol. Asterisks mark spinning sidebands, while arrows indicate additional signals originating from a monohydrate form of 2,6-DHBA.

Surprisingly, after grinding 2,6-DHBA sample with methanol or water the small intensity signals visible in the NMR spectrum of the commercial sample and originating from the monohydrate form are no longer detectable. Instead, only signals originating from the orthorhombic form are visible. Therefore, this form was designated as the one taking part in the mechanochemical reactions with LIN.

3,4-dihydroxybenzoic acid (3,4-DHBA) has only one neat crystal structure published in the CSD database, which was crystallized from the melt (CSD refcode WUYNUA), and three 
crystal structures of 3,4-DHBA monohydrates: two triclinic forms (CSD refcodes BIJDON03 ${ }^{12}$ and BIJDON04) ${ }^{3}$ and one monoclinic form (CSD refcode BIJDON05) ${ }^{13}$. The ${ }^{13} \mathrm{C}$ CPMAS NMR spectrum of commercial 3,4-DHBA shows very broad resonances (Figure S40) suggesting the presence of at least partially amorphous form. Mechanochemical grinding of this sample in the presence of water, as well as its crystallization from water yielded a form with two sets of ${ }^{13} \mathrm{C}$ resonances in the solid-state NMR spectrum (Figure S40), indicating a $Z^{\prime}=2$ structure, i.e. triclinic rhombic monohydrate form of 3,4-DHBA (BIJDON03). This form was used in all grinding experiments.

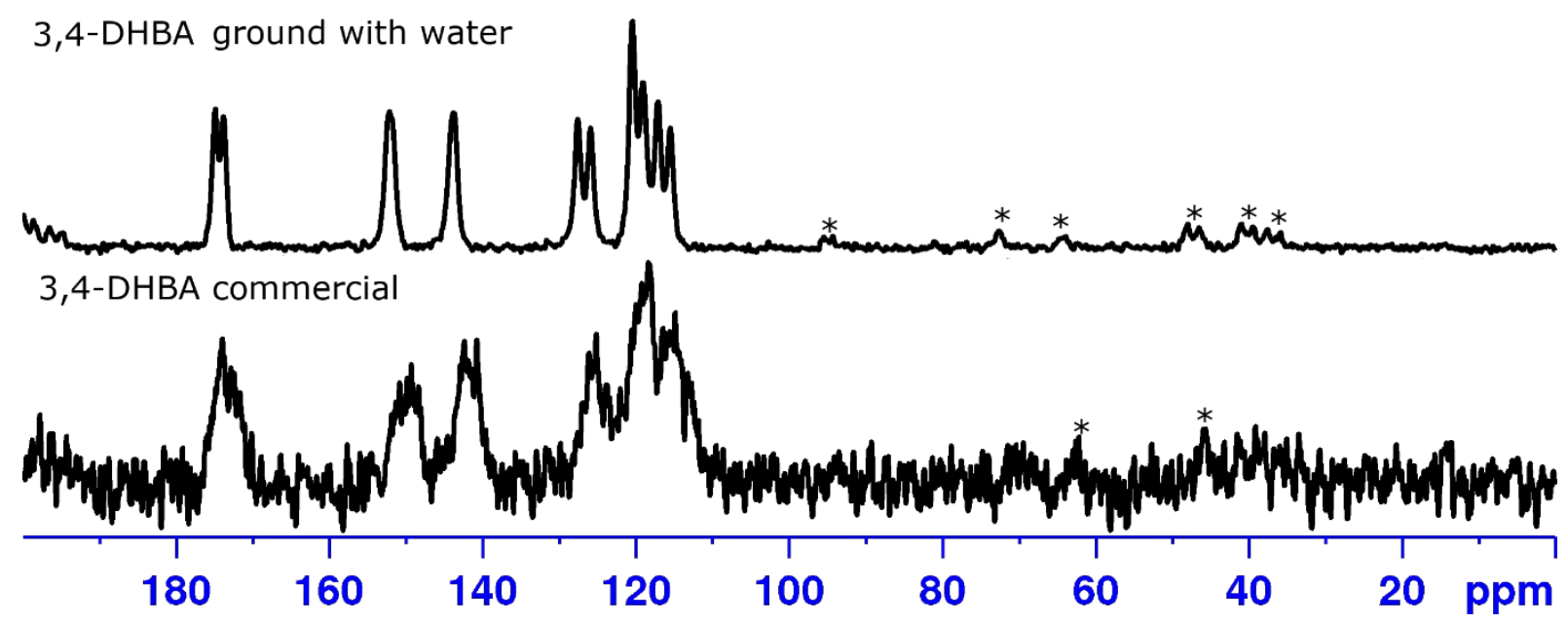

Figure S40. ${ }^{13} \mathrm{C}$ CPMAS NMR spectra of the commercial sample of 3,4-DHBA and the same sample after grinding with water.

3,5-dihydroxybenzoic acid (3,5-DHBA) has two neat polymorphic forms: form 1 (CSD refcode WUYPOW) ${ }^{3}$ and form 2 (CSD refcode WUYPOW01), ${ }^{3}$ in addition to a hemihydrate (CSD refcode OKEMAT). ${ }^{14}$ The DSC curve for the commercial sample of 3,5-DHBA do not show any endothermic events that might be associated with a loss of crystalline water (see Figure S13). Therefore this is an anhydrous form. Its ${ }^{13} \mathrm{C}$ CPMAS NMR spectrum indicates the presence of at least two sets of ${ }^{13} \mathrm{C}$ resonances (possibly three, see Figure S41), indicating that this is form 2 of 3,5-DHBA. The presence of form 2 in the commercial sample is further confirmed by the PXRD data (see Figure 41). This form was used in the grinding experiments with LIN.

Grinding form 2 of 3,5-DHBA with methanol did not change its ${ }^{13} \mathrm{C}$ CPMAS NMR spectrum, but upon grinding with water a different form of 3,5-DHBA emerged with only one set of ${ }^{13} \mathrm{C}$ resonances (in contrast to the known hemihydrate, which is a $Z^{\prime}=2$ structure). The PXRD diffractogram registered for this form indicated that this is a different from the published hemihydrate, in agreement with the NMR data. As this form does not easily yield crystals for single-crystal X-Ray measurements, establishing its crystal structure is beyond the scope of this work and will be in the future. 

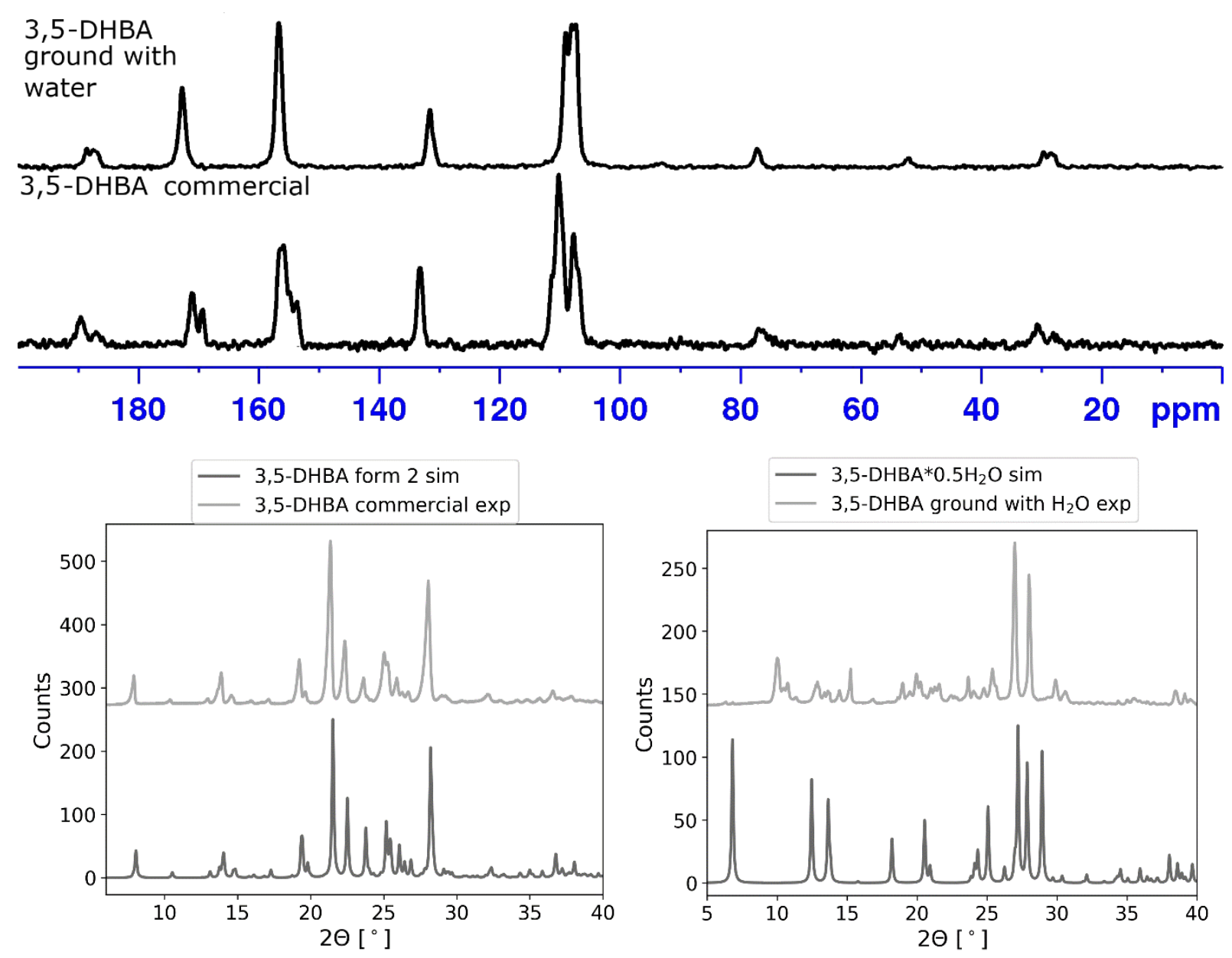

Figure S41. Upper: ${ }^{13} \mathrm{C}$ CPMAS NMR spectra registered for a commercial sample of 3,5DHBA and the same sample after grinding with methanol or water. In the case of methanol no phase change was observed to the sample, but grinding with water yielded a different phase. Lower left: experimental PXRD data for the commercial sample of 3,5-DHBA and its comparison with the simulated powder pattern for form 2 of 3,5-DHBA (WUYPOW01). Lower right: comparison of the experimental PXRD data for 3,5-DHBA sample ground with water and the simulated PXRD pattern for a known hemihydrate of 3,5-DHBA.

p-hydroxybenzoic acid (PHBA) has two neat crystal structures published in the CSD in $P 2_{1} /$ a (CSD refcode JOZZIH, form I) ${ }^{15}$ and $P 2_{1} / \mathrm{n}$ monoclinic space groups (CSD refcode JOZZIH01, form II), ${ }^{16}$ in addition to a monohydrate (CSD refcode PHBZAC02). ${ }^{17}$ The DSC registered for a commercial sample of PHBA (Figure S42) indicates that this form is not a hydrate. Grinding the commercial sample with water or methanol does not cause any changes to the phase of the commercial sample, as indicated by the ${ }^{13} \mathrm{C}$ CPMAS NMR measurements (Figure S42). The PXRD patterns for both neat polymorphs of PHBA are very similar, as both structures share some common features. Nevertheless, the comparison of the simulated PXRD data with the experimental diffractogram for commercial PHBA indicates that it is rather form I, with the positions of the diffraction peaks being closer to this form than to form II (see Figure S42). 

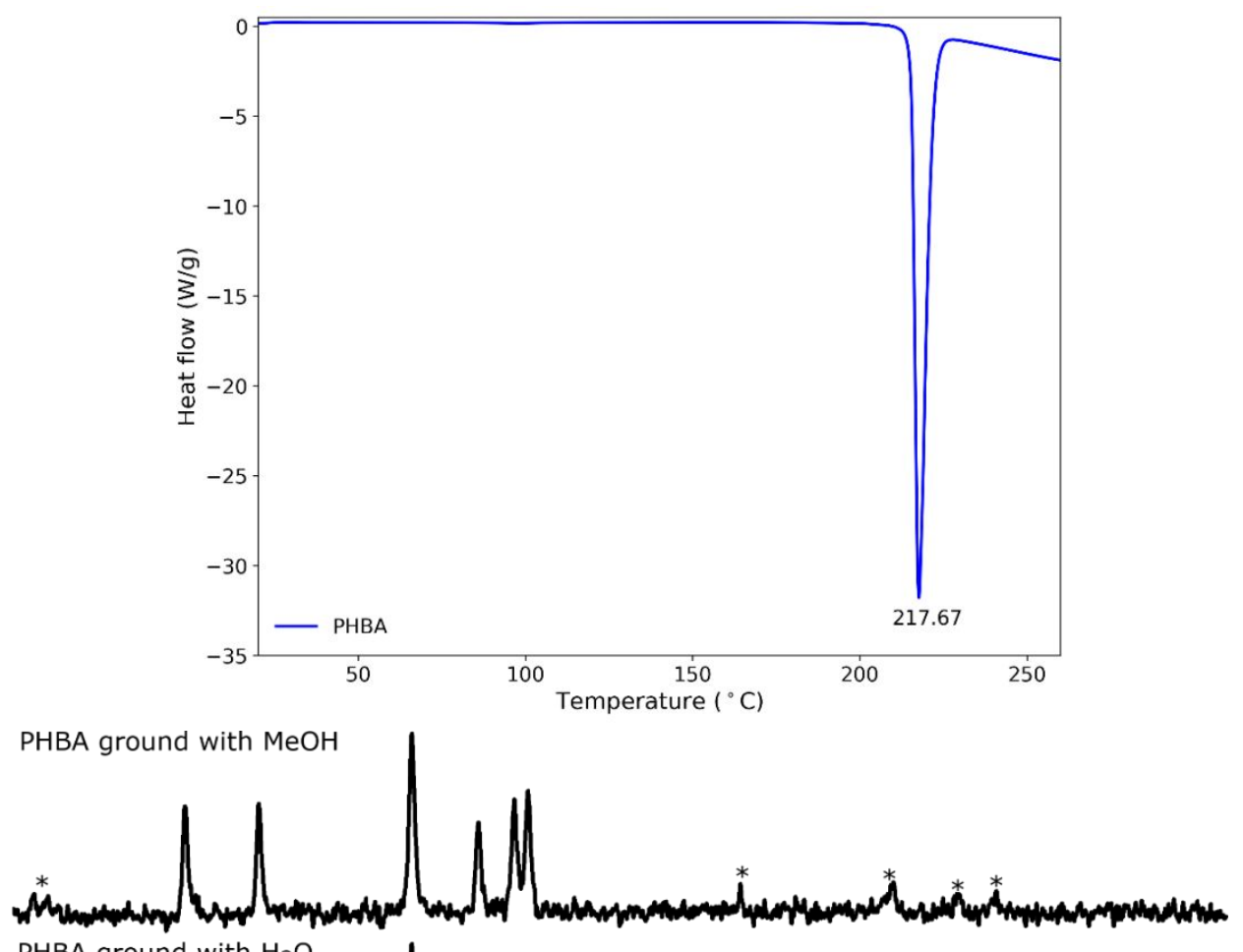
PHBA ground with $\mathrm{H}_{2} \mathrm{O}$
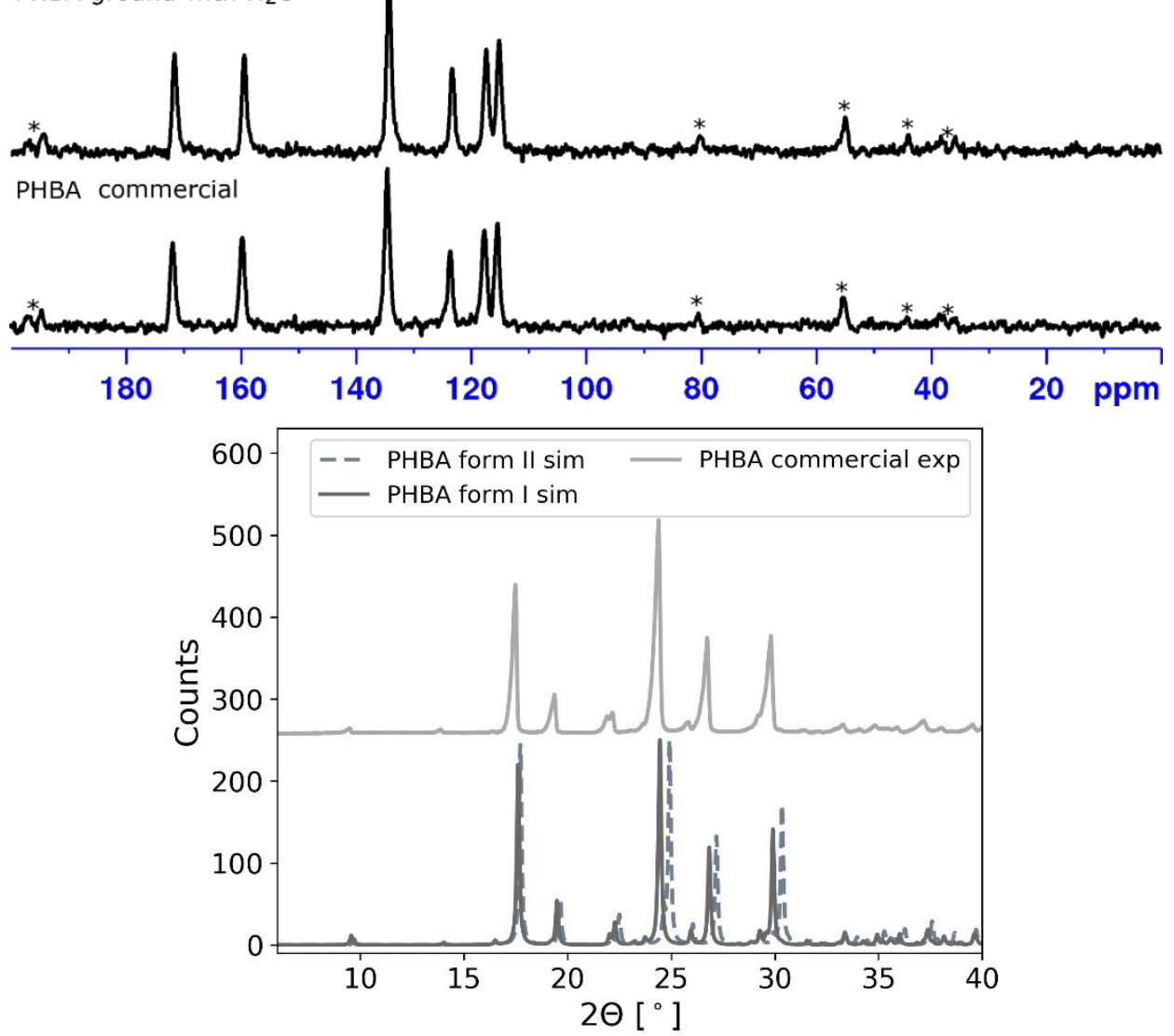

Figure S42. Upper: DSC curve for commercial sample of PHBA. Middle: ${ }^{13}$ C CPMAS NMR spectra registered for a commercial sample of PHBA and the same sample after grinding with methanol or water. No phase change was observed upon grinding. Asterisks mark spinning sidebands. Lower: experimental PXRD data registered for the commercial sample of PHBA and its comparison with the simulated PXRD patterns for two polymorphic forms of this coformer. 
p-aminobenzoic acid (PABA) has four neat polymorphic forms with known crystal structures: form alpha I (CSD refcode AMBNAC06), ${ }^{18}$ form beta IV (CSD refcode AMBNAC10),Error! Bookmark not defined. high pressure form delta (CSD refcode AMBNAC14) ${ }^{19}$ and orthorhombic form V (CSD refcode AMBNAC09). ${ }^{20}$ The DSC measurement for commercial sample of PABA shows the melting event at ca. $190{ }^{\circ} \mathrm{C}$ (Figure S14), which is in good agreement with the literature melting point for the alpha I form. The presence of this form is also indicated by the PXRD data registered for the commercial sample (see Figure S43).

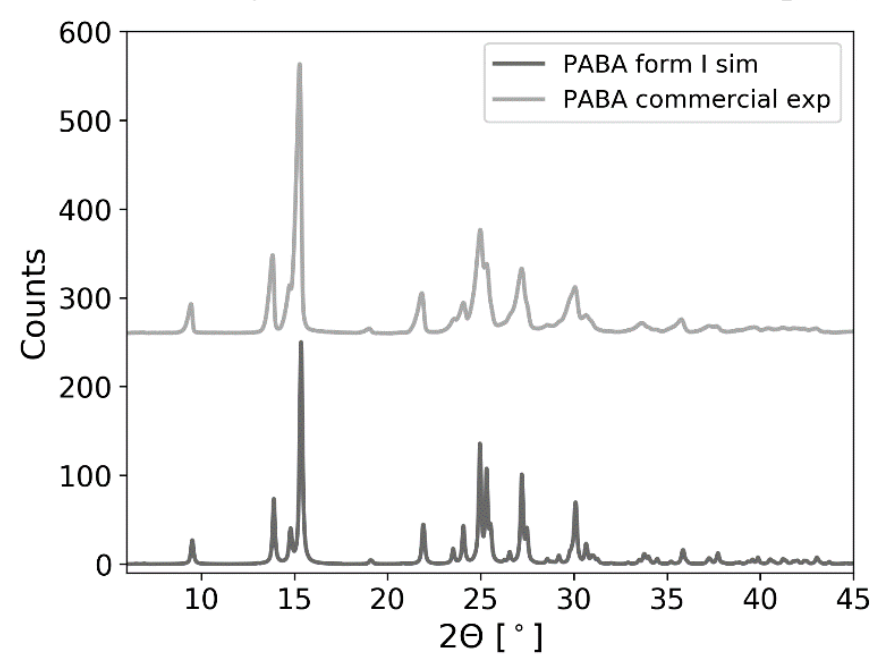

Figure S43. Experimental PXRD data registered for the commercial sample of PABA and its comparison with the simulated PXRD pattern for the $\alpha$ polymorph of PABA.

Gallic acid (GA) used for grinding experiments was in its monohydrate form. There are six polymorphs of GA monohydrate in the CSD, of which five are monoclinic polymorph and one is a triclinic polymorph. These are: $P 2_{1} / \mathrm{c}$ form I (CSD refcodes KONTIQ), ${ }^{21} P 2 / \mathrm{n}$ form II (CSD refcode KONTIQ01), ${ }^{22}$ triclinic $P$-1 form III (CSD refcode KONTIQ04), ${ }^{23} P 2_{1} / \mathrm{c}$ form IV (CSD refcode KONTIQ05), ${ }^{23} P 2_{1} / \mathrm{c}$ form V (CSD refcode KONTIQ06), ${ }^{24}$ and $P 2_{1} / \mathrm{c}$ form VI (CSD refcode KONTIQ08). ${ }^{25}$ The PXRD data indicate that the sample used for grinding experiments with LIN contains form II of GA monohydrate (see Figure S44). Upon grinding of the commercial form with water or methanol no changes to the ${ }^{13} \mathrm{C}$ CPMAS NMR spectra are observed (Figure S44), indicating a lack of a phase change.

Vanilic acid (VA) has only one known crystal structure in the CSD, with the CSD refcode CEHGUS. ${ }^{26}$ The ${ }^{13} \mathrm{C}$ CPMAS NMR spectrum registered for a commercial sample of this coformer (see Figure S25) confirms that it is crystalline, with sharp resonances indicating $Z^{\prime}=1$ structure, in agreement with the reported crystal structure. 

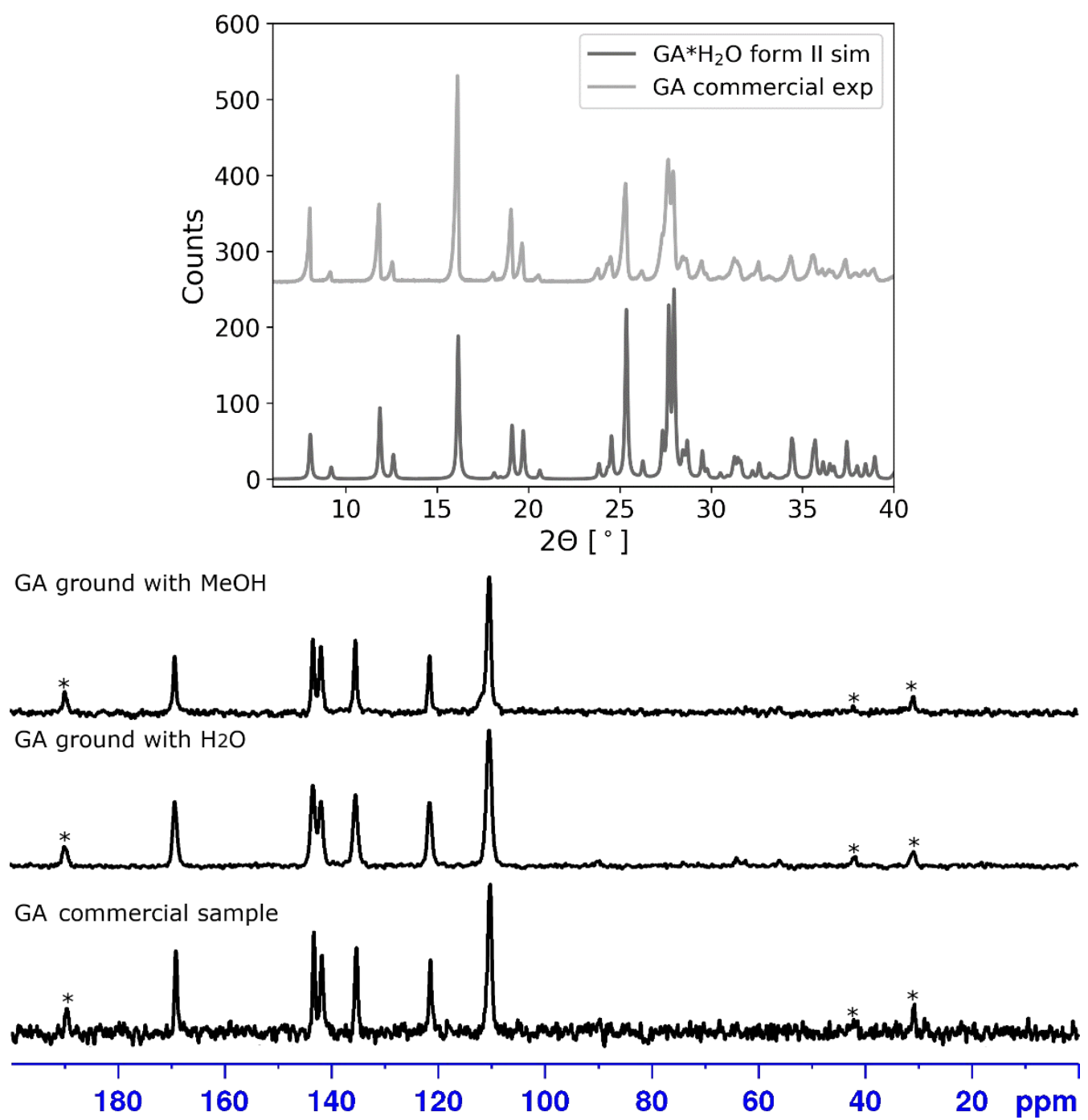

Figure S44. Upper: experimental PXRD data registered for the commercial sample of GA monohydrate and its comparison with the simulated PXRD pattern for the polymorphic form II of GA monohydrate; lower: ${ }^{13} \mathrm{C}$ CPMAS NMR spectra registered for a commercial sample of GA and the same sample after grinding with methanol or water. No phase change was observed upon grinding. Asterisks mark spinning sidebands.

Sulfanilic acid (SA) crystallizes in two neat polymorphic forms, orthorhombic $P$ ca2 1 form (CSD refcode AFAZEM) ${ }^{27}$ and monoclinic $P 2{ }_{1} / \mathrm{c}$ form (AFAZEM01), ${ }^{28}$ and in both of them it is in zwitterionic form. In addition there are four known hydrates, two monohydrates with SA as a zwitterion, a $P 2{ }_{1} / \mathrm{c}$ monoclinic form (CSD refcode SANACM) ${ }^{29}$ and a $P 2{ }_{1} 2_{1} 2_{1}$ orthorhombic form (CSD refcode SANACM01), ${ }^{30}$ as well as one monohydrate (CSD refcode ISESEG) $^{31}$ and one dihydrate (CSD refcode NESVOZ) ${ }^{32}$ with SA in its neutral form. The PXRD data for the commercial sample of SA indicates that it is a $P$ ca2 $1_{1}$ polymorph (Figure S45). Upon grinding this sample with water a phase change is observed, consistent with the formation of the $P 2_{1} / \mathrm{c}$ monohydrate form, as also indicated by the PXRD pattern registered for SA sample after grinding with water. 

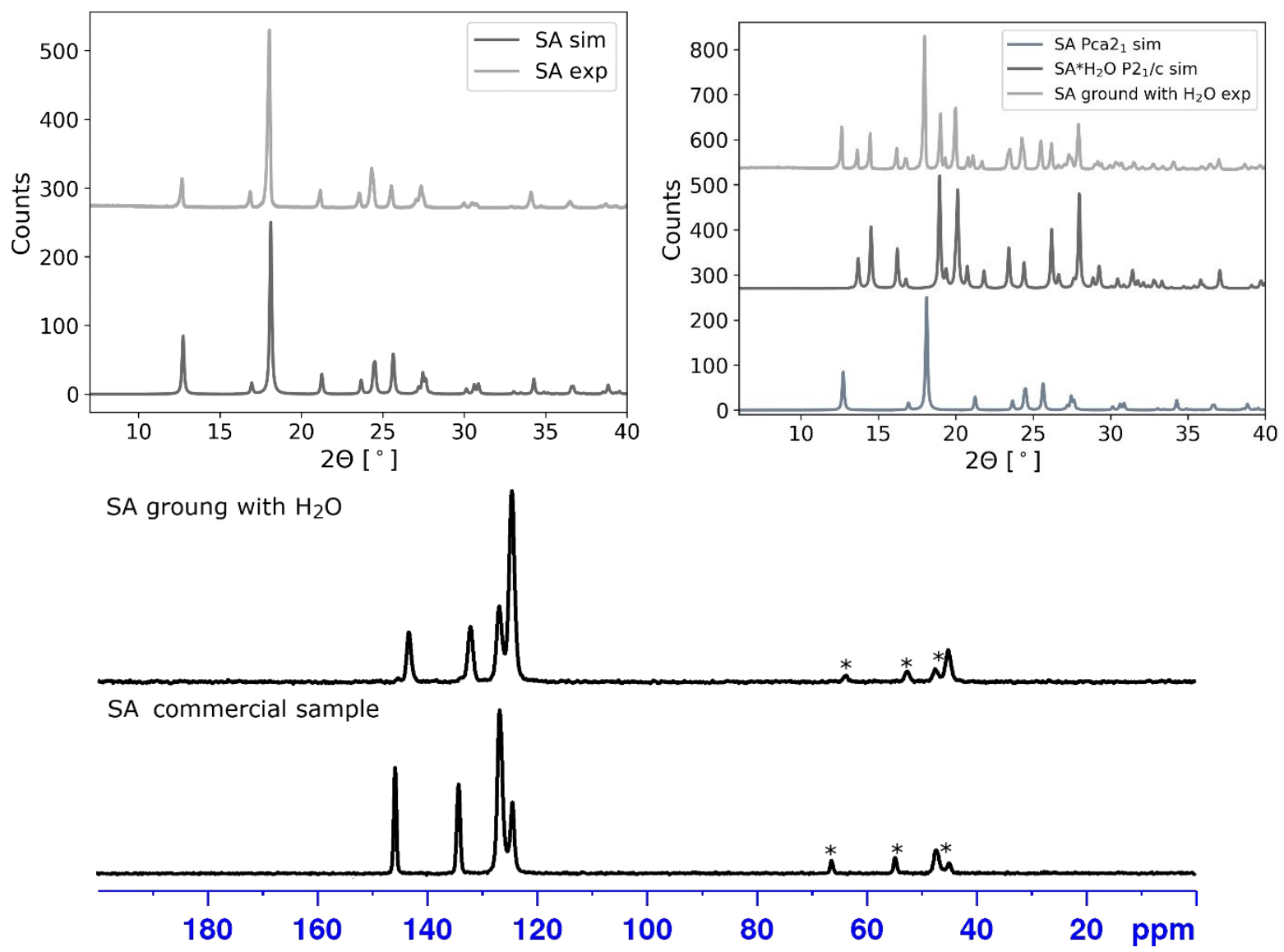

Figure S45. Upper left: experimental PXRD data registered for the commercial sample of SA and its comparison with the simulated PXRD pattern for the orthorhombic polymorph of SA (AFAZEM); upper right: experimental PXRD data registered for SA sample ground with water and its comparison with the simulated PXRD pattern for the $P 2_{1} / \mathrm{c}$ polymorph of SA monohydrate (SANACM); lower: ${ }^{13} \mathrm{C}$ CPMAS NMR spectra registered for a commercial sample of SA and the same sample after grinding with water. A phase change upon grinding is readily observable. Asterisks mark spinning sidebands.

Isonicotinic acid (INA) has only one known crystal structure in the CSD, found in triclinic $P$ 1 space group (CSD refcode ISNICA). ${ }^{33}$ The ${ }^{13} \mathrm{C}$ CPMAS NMR spectrum of the commercial sample indicates it is crystalline (see Figure S29).

Isonicotinamide (INN) is known to crystallize in as many as six neat polymorphic forms: monoclinic $P 2_{1} / \mathrm{c}$ form I (CSD refcode EHOWIH), monoclinic $P 2_{1} / \mathrm{c}$ form II (CSD refcode EHOWIH02), orthorhombic Pbca form III (CSD refcode EHOWIH03), ${ }^{34}$ monoclinic $P$ c form IV (CSD refcode EHOWIH04), ${ }^{35}$ monoclinic $P 2_{1} / \mathrm{c}$ form V (CSD refcode EHOWIH05) ${ }^{35}$ and recently discovered orthorhombic $P$ ca $2{ }_{1}$ form VI (CSD refcode EHOWIH06). ${ }^{36}$ In addition to this reach variety of neat polymorphs, there are two monoclinic crystal structures of INN monohydrates, a $P 2{ }_{1} / \mathrm{c}$ form (CSD refcode MOVTIB01) ${ }^{37}$ and $P \mathrm{c}$ form (CSD refcode MOVBIT02). ${ }^{37}$

The ${ }^{13} \mathrm{C}$ CPMAS NMR spectrum of the commercial sample of INN indicates that this sample contains one of the three forms having $Z^{\prime}=1$ structure (see Figure S30), i.e. form I, form III or form V, while PXRD data registered for this sample proves the presence of form I (Figure S46). 
Grinding INN with water or methanol does not cause any changes to its phase according to the ${ }^{13} \mathrm{C}$ CPMAS NMR spectrum.

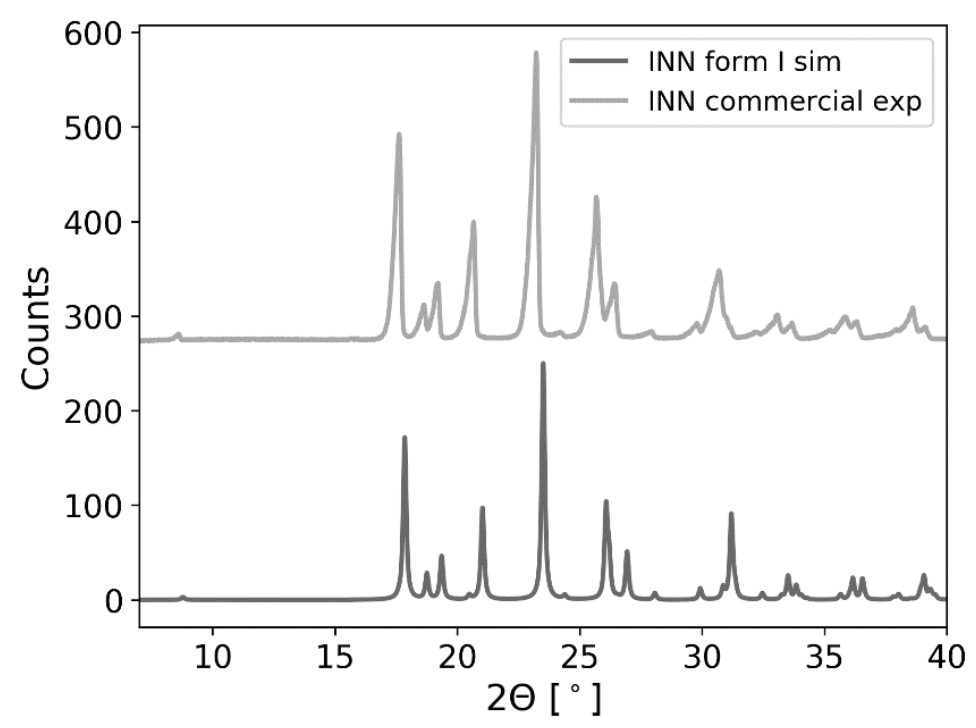

Figure S46. Experimental PXRD data registered for the commercial sample of INN and its comparison with the simulated PXRD pattern for the polymorphic form II of INN (EHOWIH).

Ibuprofen (IBU) commercial sample used for grinding experiments is a racemic mixture of $\mathrm{R} / \mathrm{S}$ forms. The racemate of IBU is known to crystallize in either of the two $P 2_{1} / \mathrm{c}$ monoclinic forms, form 1 (CSD refcode IBPRAC01) ${ }^{38}$ and form 2 (CSD refcode IBPRAC04). ${ }^{39}$ The ${ }^{13} \mathrm{C}$ CPMAS NMR spectrum registered for the commercial sample of IBU (see Figure S31) is consistent with its form 1 .

L-tyrosine (TYR) has in the CSD only one structure, that of $P 2{ }_{1} 2{ }_{1}{ }_{1}$ orthorhombic form (CSD refcode LTYROS11). ${ }^{40}$ The ${ }^{13} \mathrm{C}$ CPMAS spectrum of the commercial sample (see Figure S32) confirms it is in a crystalline state.

L-proline (PRO) crystallizes in two neat polymorphic forms, orthorhombic $P 2{ }_{1} 2_{1} 2_{1}$ form I (CSD refcode PROLIN02) ${ }^{41}$ and triclinic $P 1$ form II (CSD refcode PROLIN04), ${ }^{41}$ in addition to a monohydrate (CSD refcode RUWGEV) ${ }^{42}$ crystallizing in monoclinic $C 2$ space group. The two neat forms of PRO are easily discernible by ${ }^{13} \mathrm{C}$ CPMAS NMR, as the second form is a $Z$ '=2 structure. The solid-state NMR spectrum register for the commercial sample indicate that it is a $Z$ ' $=1$ structure and therefore most probably form I. Grinding this form in the presence of water or methanol yields a new form, with only small amounts of the commercial form present in the sample after the grinding. This new form was identified on the basis of its PXRD pattern as PRO monohydrate (see comparison of PXRD diffractograms in Figure S47). Interestingly, grinding PRO alone also leads to the formation of small amounts of the same monohydrate form, as shown by the ${ }^{13} \mathrm{C}$ CPMAS NMR spectra registered for the commercial sample and this same sample after grinding it alone, as well as in the presence of methanol (Figure S47). Also PXRD data registered for PRO ground in a ball mill alone shows the presence of a small amount 
of PRO monohydrate. As a result both forms, PRO form I and PRO monohydrate should be considered as possible forms taking part in the mechanochemical reactions.
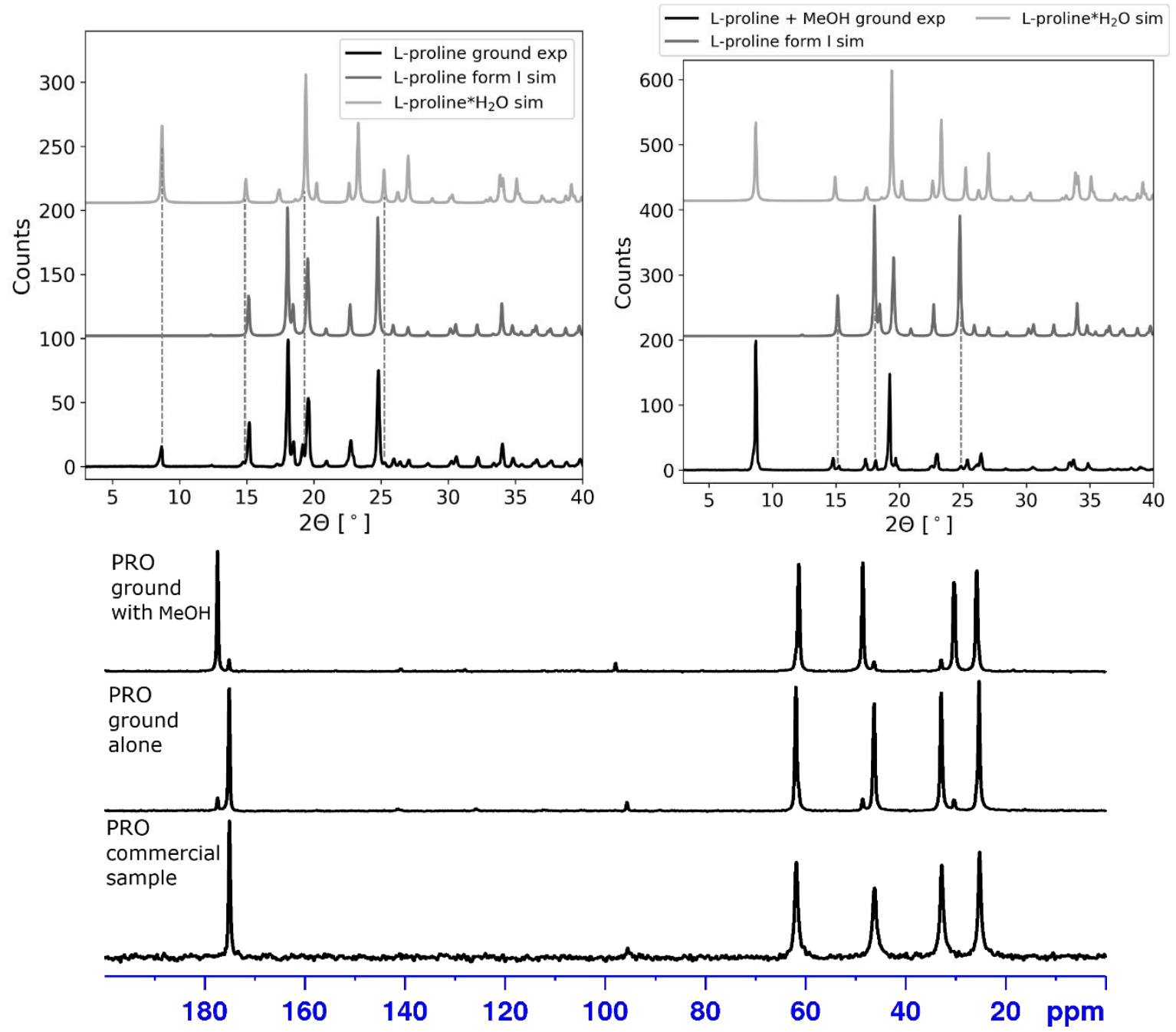

Figure S47. Upper: a comparison of the experimental PXRD data registered for the commercial sample of PRO ground alone for $1 \mathrm{~h}$ in a ball mill (left) and for the commercial sample of PRO ground in a ball mill with methanol for $1 \mathrm{~h}$ with the simulated powder patterns for PRO form I (PROLIN02) and PRO monohydrate (RUWGEV) (right); lower: ${ }^{13} \mathrm{C}$ CPMAS NMR spectra registered for a commercial sample of PRO and the same sample after grinding it alone or with methanol in a ball mill. In both cases after grinding a new form emerges, with the difference in the quantity of this form.

Malonic acid (MAL) can crystallize in either of the three polymorphic forms: orthorhombic $P b c n$ form alpha, for which the only published crystal structure contains perdeuterated compound (CSD refcode MALNAC03), triclinic $P-1$ form beta (CSD refcode MALNAC02) and monoclinic $P 2_{1} / \mathrm{n}$ epsilon form (CSD refcode MALNAC10). The PXRD data indicates that the commercial sample contains form $\beta$ of MAL (Figure S48). 


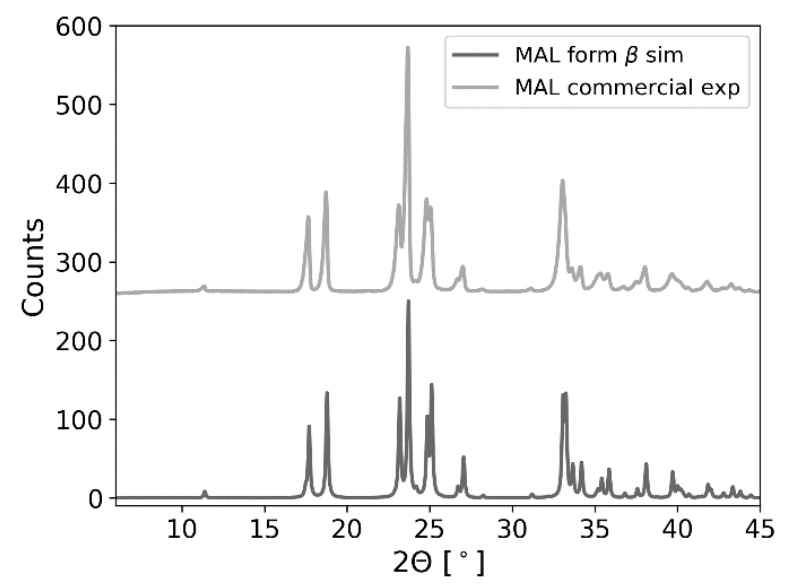

Figure S48. Experimental PXRD data registered for the commercial sample of MAL and its comparison with the simulated PXRD pattern for the polymorphic form $\beta$ of MAL (MALNAC02).

Barbituric acid (BARB) can crystallize as one of the four known polymorphic forms, monoclinic $P 2_{1} / \mathrm{c}$ form I (CSD refcode BARBAC01), ${ }^{43}$ monoclinic $P 2_{1} / \mathrm{c}$ form II with two BARB molecules in an asymmetric unit cell (CSD refcode BARBAC02), ${ }^{43}$ high temperature monoclinic $C 2 / \mathrm{c}$ form III (CSD refcode BARBAC03), ${ }^{44}$ and monoclinic $P 2_{1} / \mathrm{n}$ form IV (CSD refcode IYAQOP) ${ }^{45}$ In the forms I-III BARB is present in its keto form, whereas in form IV it exist as an enol. The commercial sample contains form II of BARB, which is evidenced from the characteristic ${ }^{13} \mathrm{C}$ CPMAS NMR spectrum of this sample (Figure S49), showing two wellseparated resonances for $\mathrm{CH}_{2}$ group, a feature characteristic for form II. Although there are reports in the literature that grinding BARB leads to the formation of form IV, we did not observed such effect after $1 \mathrm{~h}$ of grinding of BARB alone or in the presence of methanol. However, upon grinding with water, a new form was formed, with the ${ }^{13} \mathrm{C}$ CPMAS NMR spectrum (Figure S49) consistent with the orthorhombic Pnma polymorph of BARB dihydrate $\left(\mathrm{CSD}\right.$ refcode BARBAD03) ${ }^{46}$ (the monoclinic $P 2_{1} / \mathrm{n}$ dihydrate polymorph of BARB, BARBAD02, is a low-temperature form, stable below $220 \mathrm{~K}) .{ }^{46}$

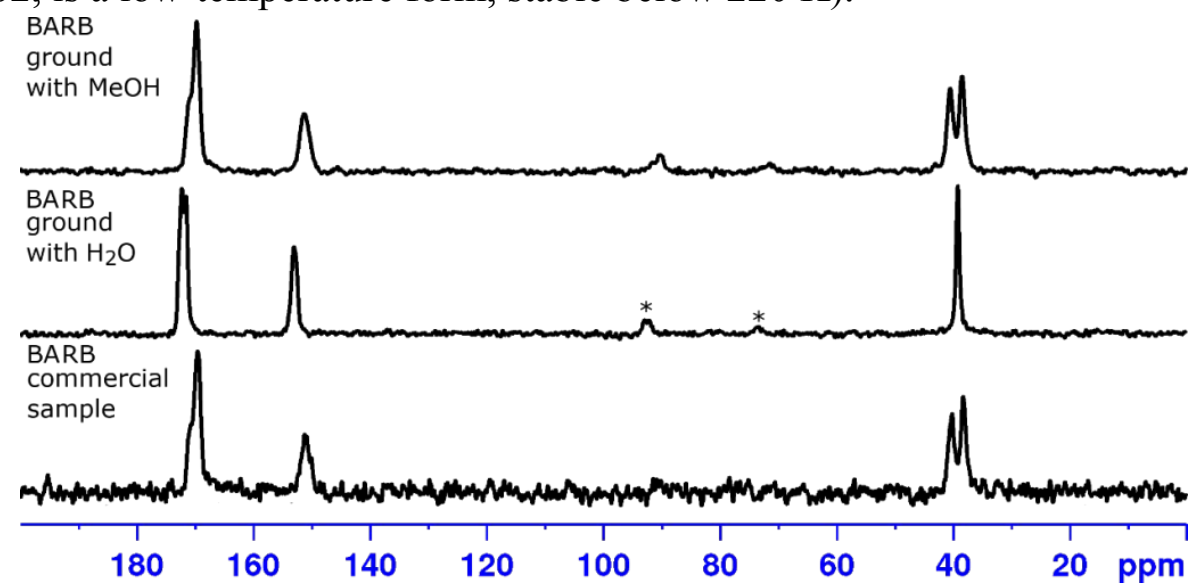

Figure S49. ${ }^{13} \mathrm{C}$ CPMAS NMR spectra registered for a commercial sample of BARB and the same sample after grinding with methanol or water. No phase change was observed upon grinding with methanol, grinding with water yielded BARB dihydrate. Asterisks mark spinning sidebands. 
Table S2 summarizes the data on the known polymorphic forms of the studied coformers and on their hydrates and indicates which forms were accounted for as taking part in the mechanochemical grinding with LIN.

Table S2. Summary of the known polymorphic forms of the coformers used in this study. Bolded positions indicate crystal forms taking part in the mechanochemical grinding.

\begin{tabular}{|c|c|c|c|c|}
\hline coformer & CSD refcode & SG & Z' & form \\
\hline BA & BENZAC13 & $P 2_{1} / \mathrm{c}$ & 1 & monoclinic \\
\hline \multirow[t]{2}{*}{ 2,3-DHBA } & CACDAM & $P-1$ & 2 & triclinic \\
\hline & CACDAM01 & $P 2_{1} / \mathrm{n}$ & 1 & monoclinic \\
\hline \multirow[t]{4}{*}{ 2,4-DHBA } & ZZZEEU01 & $P 2_{1} / \mathbf{n}$ & 1 & form II \\
\hline & ZZZEEU08 & $P 2_{1} / \mathrm{a}$ & 1 & form I \\
\hline & QIVTUK01 & $P-1$ & 2 & hemihydrate \\
\hline & YUXGUV & $P-1$ & 1 & monohydrate \\
\hline \multirow[t]{2}{*}{ 2,5-DHBA } & BESKAL03 & $P 2_{1} / \mathrm{n}$ & 1 & form II - DSC \\
\hline & BESKAL02 & $P 2_{1} / \mathrm{c}$ & 1 & form I \\
\hline \multirow[t]{3}{*}{ 2,6-DHBA } & LEZJAB & Pna2 $_{1}$ & 1 & orthorhombic \\
\hline & LEZJAB01 & $P 2_{1} / \mathrm{c}$ & 1 & monoclinic \\
\hline & LEZJEF & Pnma & 0.5 & monohydrate \\
\hline \multirow[t]{4}{*}{ 3,4-DHBA } & WUYNUA & $P-1$ & 3 & crystallized from the melt \\
\hline & BIJDON03 & $P-1$ & 2 & triclinic rhombic monohydrate \\
\hline & BIJDON04 & $P 2_{1} / \mathrm{n}$ & 1 & monoclinic needle monohydrate \\
\hline & BIJDON05 & $P-1$ & 4 & triclinic 2 monohydrate \\
\hline \multirow[t]{3}{*}{ 3,5-DHBA } & WUYPOW & $C 2 / \mathrm{c}$ & 0.5 & form 1 \\
\hline & WUYPOW01 & $C 2 / \mathrm{c}$ & 3 & form 2 \\
\hline & OKEMAT & $P 2_{1}$ & 2 & hemihydrate \\
\hline \multirow[t]{3}{*}{ PHBA } & JOZZIH & $P 2_{1} / \mathbf{a}$ & 1 & monoclinic form I \\
\hline & JOZZIH01 & $P 2_{1} / \mathrm{n}$ & 1 & monoclinic form II \\
\hline & PHBZAC02 & & & monohydrate \\
\hline \multirow[t]{4}{*}{ PABA } & AMBNAC06 & $P 2_{1} / \mathbf{n}$ & 2 & form alpha I \\
\hline & AMBNAC10 & $P 2_{1} / \mathrm{n}$ & 1 & form beta IV \\
\hline & AMBNAC09 & $P$ na $2_{1}$ & 2 & form $\mathrm{V}$ \\
\hline & AMBNAC14 & Pn & 1 & high pressure form delta \\
\hline
\end{tabular}




\begin{tabular}{|c|c|c|c|c|}
\hline \multirow[t]{6}{*}{ GA } & KONTIQ & $P 2_{1} / \mathrm{c}$ & 1 & monohydrate, $P 2_{1} / \mathrm{c}$ form I \\
\hline & KONTIQ01 & $P 2 / \mathbf{n}$ & 1 & monohydrate $P 2 / n$ form II \\
\hline & KONTIQ04 & $P-1$ & 4 & monohydrate triclinic form III \\
\hline & KONTIQ05 & $P 2_{1} / \mathrm{c}$ & 1 & monohydrate monoclinic form IV \\
\hline & KONTIQ06 & $P 2_{1} / \mathrm{c}$ & 1 & monohydrate form $\mathrm{V}$ \\
\hline & KONTIQ08 & $P 2_{1} / \mathrm{c}$ & 3 & monohydrate form VI \\
\hline VA & CEHGUS & $P 2_{1} / \mathrm{c}$ & 1 & \\
\hline \multirow[t]{6}{*}{ SA } & AFAZEM & Pca2 $_{1}$ & 1 & Pca2 $_{1}$ \\
\hline & AFAZEM01 & $P 2_{1} / \mathrm{c}$ & 1 & $P 2_{1} / \mathrm{c}$ \\
\hline & ISESEG & Pbca & 1 & monohydrate \\
\hline & NESVOZ & $P 2_{1} / \mathrm{n}$ & 1 & dihydrate \\
\hline & SANACM & $P 2_{1} / \mathbf{c}$ & 1 & zwitterionic monohydrate $P 2_{1} / \mathrm{c}$ form \\
\hline & SANACM01 & $P 2_{1} 2_{1} 2_{1}$ & 1 & zwitterionic monohydrate $P 2{ }_{1} 2{ }_{1}{ }_{1}$ form \\
\hline INA & ISNICA & $P-1$ & 1 & \\
\hline \multirow[t]{8}{*}{ INN } & EHOWIH & $P 2_{1} / c$ & 1 & form I \\
\hline & EHOWIH02 & $P 2_{1} / \mathrm{c}$ & 2 & form II \\
\hline & EHOWIH03 & Pbca & 1 & form III (Iso3) \\
\hline & EHOWIH04 & $P \mathrm{c}$ & 3 & monoclinic $P \mathrm{c}$ form IV \\
\hline & EHOWIH05 & $P 2_{1} / \mathrm{c}$ & 1 & monoclinic $P 2_{1} / \mathrm{c}$ form $\mathrm{V}$ \\
\hline & EHOWIH06 & $P c a 2_{1}$ & 2 & $P$ ca $2_{1}$ form VI \\
\hline & MOVTIB01 & $P 2_{1} / \mathrm{n}$ & 2 & monohydrate \\
\hline & MOVTIB02 & $P \mathrm{c}$ & 8 & monohydrate \\
\hline \multirow[t]{2}{*}{ IBU } & IBPRAC01 & $P 2_{1} / \mathrm{c}$ & 1 & racemate form 1 \\
\hline & IBPRAC04 & $P 2_{1} / \mathrm{c}$ & 1 & racemate form 2 \\
\hline TYR & LTYROS11 & $P 2_{1} 2_{1} 2_{1}$ & 1 & \\
\hline \multirow[t]{3}{*}{ PRO } & PROLIN02 & $P 2_{1} 2_{1} 2_{1}$ & 1 & zwitterionic form I \\
\hline & PROLIN04 & $P 1$ & 2 & zwitterionic high-temp. form II \\
\hline & RUWGEV & $C 2$ & 1 & zwitterionic monohydrate \\
\hline \multirow[t]{3}{*}{ MAL } & MALNAC02 & $P-1$ & 1 & beta triclinic \\
\hline & MALNAC03 & Pbcn & 0.5 & alpha orthorhombic \\
\hline & MALNAC10 & $P 2_{1} / \mathrm{n}$ & 3 & epsilon \\
\hline \multirow[t]{6}{*}{ BARB } & BARBAC01 & $P 2_{1} / \mathrm{c}$ & 1 & form I \\
\hline & BARBAC02 & $P 2_{1} / \mathrm{c}$ & 2 & form II \\
\hline & BARBAC03 & $C 2 / \mathrm{c}$ & 0.5 & form III \\
\hline & IYAQOP-03 & $P 2_{1} / \mathrm{n}$ & 1 & enol form IV \\
\hline & BARBAD03 & Pnma & 0.5 & dihydrate orthorhombic form \\
\hline & BARBAD02 & $P 2_{1} / \mathrm{n}$ & 1 & dihydrate monoclinic low-temp. form \\
\hline
\end{tabular}


Linezolid (LIN) has two known polymorphic forms: $P 2_{1} 2_{2} 2_{1}$ form II $\left(Z^{\prime}=1\right)$ and $P-1$ form III $\left(Z^{\prime}=2\right.$, sometimes also referred to in the literature as form IV). Figure S50 shows the ${ }^{13} \mathrm{C}$ CPMAS NMR spectra of these two forms, together with the NMR spectra of form III after 30 and 60 minutes of grinding in a ball mill. It is clear, that during grinding a phase transition LIN III -> LIN II takes place, indicating higher room temperature stability of form II. After $30 \mathrm{~min}$ of grinding signals originating from LIN form II start to appear in the NMR spectrum but form III is still a dominant form, while after 60 minutes the resonances from LIN form III are no longer detectable.

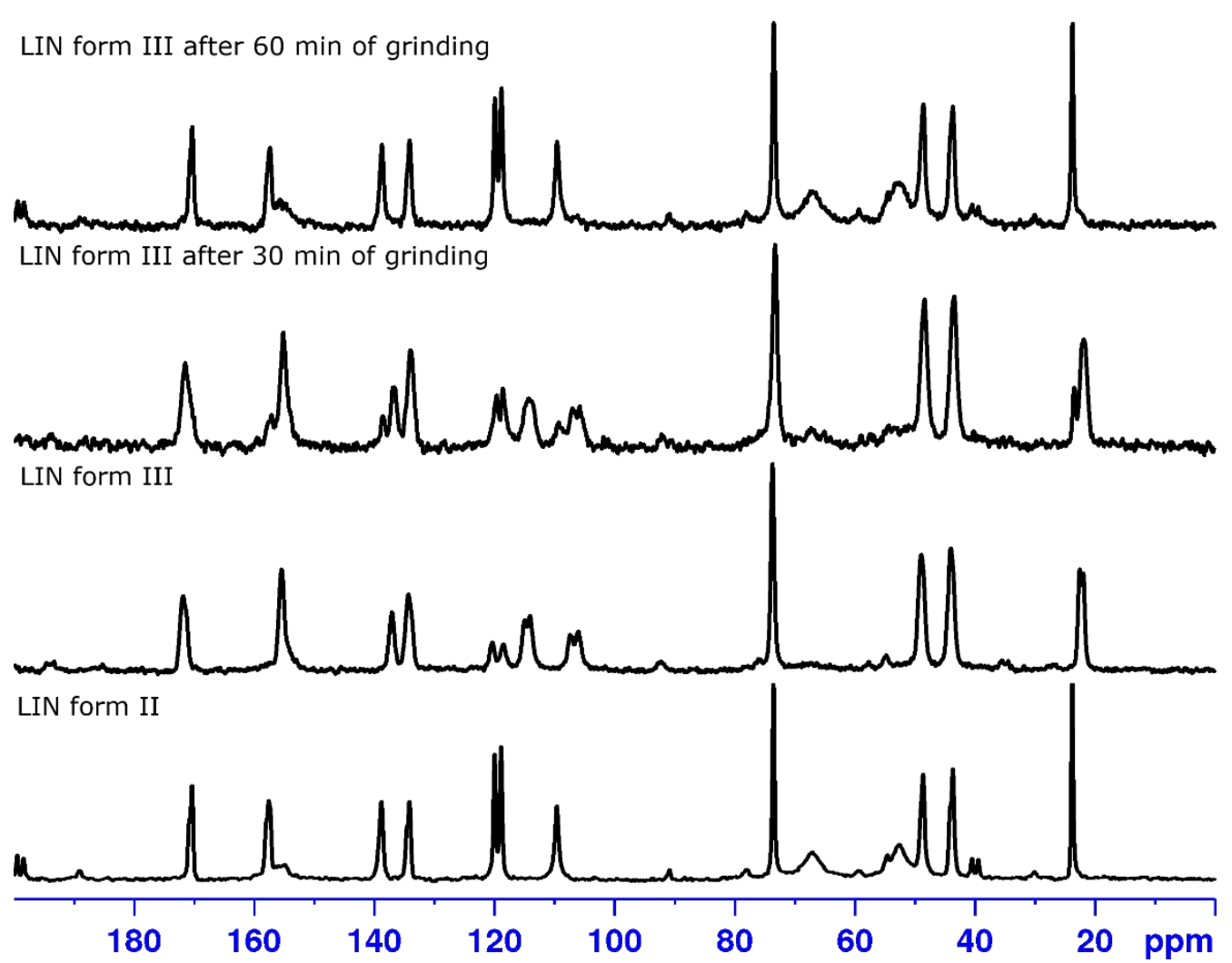

Figure S50. ${ }^{13} \mathrm{C}$ CPMAS NMR spectra of LIN form II and form III, as well as LIN form III after 30 and $60 \mathrm{~min}$ of grinding in a ball mill. The spectra were registered with a repetition time of $120 \mathrm{~s}$.

\section{Numerical values from CASTEP and MEP calculations}

Table S3. Total, intramolecular and intermolecular energies of LIN_II and LIN_III (in kJ/mol) calculated under periodic boundary conditions at PBE-D2 level of theory and expressed per one LIN molecule.

\begin{tabular}{llll}
\hline Crystal form & $E^{\text {total }}$ & E $^{\text {intramolecular }}$ & $E^{\text {intermolecular }}$ \\
\hline LIN_II & -588031 & -587709 & -321 \\
\hline LIN_III_1 & -588030 & -587718 & -314 \\
LIN_III_2 & & -587713 & \\
\hline
\end{tabular}


Table S4. Numerical $\alpha$ and $\beta$ values (in $\mathrm{kJ} / \mathrm{mol}$ ) obtained from MEP calculations.

\begin{tabular}{|c|c|c|c|c|c|c|c|c|c|c|}
\hline \multirow[t]{2}{*}{ molecule } & \multicolumn{5}{|c|}{$\alpha$ values } & \multicolumn{5}{|c|}{$\beta$ values } \\
\hline & 1 & 2 & 3 & 4 & 5 & 1 & 2 & 3 & 4 & 5 \\
\hline $\mathrm{BA}$ & 3.64 & 1.16 & 1.12 & 1.08 & 0.90 & 5.04 & 1.41 & 0.62 & 0.59 & 0.00 \\
\hline PHBA-1 & 4.35 & 3.49 & 1.60 & 0.99 & 0.87 & 5.38 & 2.29 & 1.65 & 0.64 & 0.00 \\
\hline PHBA-2 & 4.37 & 3.49 & 1.64 & 0.99 & 0.94 & 5.50 & 2.31 & 1.55 & 0.68 & 0.00 \\
\hline 2,6-DHBA-1 & 5.51 & 2.08 & 1.91 & 1.39 & 0.97 & 6.43 & 4.54 & 3.72 & 0.47 & 0.29 \\
\hline 2,6-DHBA-2 & 4.77 & 2.22 & 1.21 & 1.08 & 0.86 & 3.52 & 2.80 & 2.33 & 0.84 & 0.81 \\
\hline 3,4-DHBA & 4.89 & 3.46 & 3.04 & 1.69 & 0.93 & 5.80 & 3.51 & 1.64 & 0.74 & 0.44 \\
\hline 2,5-DHBA & 4.13 & 4.04 & 1.32 & 1.13 & 1.00 & 3.73 & 3.02 & 2.77 & 0.71 & 0.67 \\
\hline 2,4-DHBA & 4.34 & 3.94 & 1.59 & 1.07 & 1.03 & 3.90 & 3.40 & 2.54 & 1.05 & 0.75 \\
\hline 2,3-DHBA & 4.21 & 2.77 & 1.48 & 1.02 & 0.96 & 3.31 & 2.67 & 1.20 & 0.95 & 0.81 \\
\hline 3,5-DHBA-1 & 4.08 & 3.95 & 3.80 & 1.21 & 0.87 & 4.48 & 2.89 & 2.80 & 1.07 & 0.66 \\
\hline 3,5-DHBA-2 & 4.15 & 4.07 & 3.68 & 1.41 & 1.24 & 5.29 & 3.12 & 2.29 & 1.16 & 0.77 \\
\hline 3,5-DHBA-3 & 4.12 & 4.02 & 3.64 & 1.43 & 0.87 & 4.70 & 2.83 & 2.38 & 1.66 & 0.71 \\
\hline GA-1 & 4.61 & 3.62 & 3.50 & 3.01 & 0.90 & 5.02 & 3.36 & 1.90 & 0.82 & 0.78 \\
\hline GA-2 & 4.52 & 3.96 & 3.60 & 2.99 & 1.12 & 5.42 & 4.91 & 1.32 & 0.67 & 0.64 \\
\hline GA-3 & 4.73 & 3.64 & 3.53 & 2.98 & 1.19 & 5.74 & 3.64 & 1.27 & 0.85 & 0.83 \\
\hline GA-4 & 4.64 & 3.84 & 3.60 & 3.01 & 1.24 & 5.48 & 5.01 & 1.23 & 0.69 & 0.63 \\
\hline VA & 3.46 & 3.03 & 1.47 & 1.26 & 0.86 & 5.71 & 2.97 & 1.36 & 0.89 & 0.30 \\
\hline PABA & 3.19 & 2.90 & 2.89 & 1.23 & 1.19 & 6.29 & 2.15 & 2.05 & 1.21 & 0.00 \\
\hline SA & 4.04 & 3.27 & 3.26 & 1.54 & 1.52 & 6.16 & 6.06 & 3.13 & 1.26 & 0.86 \\
\hline INA & 4.14 & 1.22 & 1.10 & 1.05 & 1.01 & 5.48 & 3.88 & 0.78 & 0.00 & 0.00 \\
\hline INN & 3.42 & 2.85 & 1.74 & 1.13 & 0.95 & 5.93 & 5.76 & 0.61 & 0.38 & 0.00 \\
\hline IBU-1 & 3.53 & 0.95 & 0.92 & 0.89 & 0.83 & 4.95 & 2.30 & 1.27 & 1.04 & 0.00 \\
\hline IBU-2 & 3.52 & 0.98 & 0.95 & 0.91 & 0.89 & 4.95 & 2.31 & 1.27 & 0.10 & 0.00 \\
\hline IBU-3 & 3.59 & 0.90 & 0.89 & 0.87 & 0.85 & 5.47 & 1.50 & 1.35 & 1.53 & 0.00 \\
\hline TYR* & 4.07 & 2.74 & 1.85 & 1.66 & 1.57 & 7.70 & 5.38 & 3.03 & 1.09 & 0.00 \\
\hline
\end{tabular}




\begin{tabular}{|c|c|c|c|c|c|c|c|c|c|c|}
\hline PRO* & 2.69 & 1.57 & 1.21 & 1.19 & 1.09 & 8.36 & 5.60 & 0.00 & 0.00 & 0.00 \\
\hline MAL & 4.10 & 3.83 & 1.44 & 1.34 & 0.00 & 4.74 & 4.36 & 1.91 & 1.26 & 0.00 \\
\hline BARB & 3.36 & 3.36 & 1.78 & 1.75 & 0.00 & 3.71 & 3.70 & 3.57 & 0.00 & 0.00 \\
\hline LIN-1 & 2.83 & 1.78 & 1.69 & 1.32 & 1.11 & 6.47 & 5.70 & 4.42 & 2.31 & 2.27 \\
\hline LIN-2 & 2.82 & 1.65 & 1.61 & 1.54 & 1.32 & 6.43 & 5.88 & 4.40 & 2.43 & 1.81 \\
\hline $\mathrm{LIN}-3 / 5^{* *}$ & 3.71 & 1.77 & 1.62 & 1.32 & 1.32 & 6.86 & 5.74 & 4.43 & 3.94 & 2.47 \\
\hline LIN-4 & 2.82 & 1.78 & 1.69 & 1.59 & 1.52 & 6.48 & 5.74 & 4.41 & 2.32 & 2.27 \\
\hline
\end{tabular}

Table S5. Energy values (in $\mathrm{kJ} / \mathrm{mol}$ of molecules) obtained from calculations under periodic boundary conditions at PBE-D2 level of theory for the crystal forms of the coformers taking part in mechanochemical reaction with LIN.

\begin{tabular}{|c|c|c|c|c|}
\hline coformer & $\begin{array}{l}\text { crystal form } \\
\text { (CSD refcode) }\end{array}$ & $\mathrm{E}^{\text {total }}$ & $E^{\text {intramolecular }}$ & $E^{\text {intermolecular }}$ \\
\hline BA & $\begin{array}{l}P 2_{1} / \mathrm{c} \\
(\mathrm{BENZAC} 13)\end{array}$ & -200617 & -200503 & -115 \\
\hline $\begin{array}{l}3,4-\mathrm{DHBA} \\
{ }^{*} \mathrm{H}_{2} \mathrm{O}\end{array}$ & $\begin{array}{l}P-1 \text { triclinic } \\
\text { (BIJDON03) }\end{array}$ & -165441 & -165326 & -115 \\
\hline 2,3-DHBA & $\begin{array}{l}P-1 \text { form } \\
\text { (CACDAM) }\end{array}$ & -285345 & -285218 & -127 \\
\hline $\mathrm{GA} * \mathrm{H}_{2} \mathrm{O}$ & $\begin{array}{l}P 2 / \mathrm{n} \text { form II } \\
(\mathrm{KONTIQ} 01)\end{array}$ & -186624 & -186496 & -128 \\
\hline 2,6-DHBA & $\begin{array}{l}P \text { na2 }{ }_{1} \text { form } \\
\text { (LEZJAB) }\end{array}$ & -285350 & -285217 & -134 \\
\hline 2,5-DHBA & $\begin{array}{l}P 2_{1} / \mathrm{c} \text { form I } \\
(\text { BESKAL02) }\end{array}$ & -285346 & -285208 & -138 \\
\hline PABA & $\begin{array}{l}P 2_{1} / \mathrm{n} \text { form } \alpha \\
(\mathrm{AMBNAC} 06)\end{array}$ & -228922 & -228779 & -143 \\
\hline VA & $\begin{array}{l}P 2_{1} / \mathrm{c} \text { form } \\
(\mathrm{CEHGUS})\end{array}$ & -303639 & -303496 & -144 \\
\hline PHBA & $\begin{array}{l}P 2_{1} / \mathrm{a} \text { form I } \\
(\mathrm{JOZZIH})\end{array}$ & -242991 & -242847 & -144 \\
\hline 2,4-DHBA & $\begin{array}{l}P 2_{1} / \mathrm{n} \text { form II } \\
\text { (ZZZEEU01) }\end{array}$ & -285369 & -285224 & -145 \\
\hline MAL & $P-1$ form $\beta$ & -221416 & -221268 & -148 \\
\hline
\end{tabular}




\begin{tabular}{|c|c|c|c|c|}
\hline & (MALNAC02) & & & \\
\hline IBU & $\begin{array}{l}P 2_{1} / \mathrm{c} \text { racemate form I } \\
(\text { IBPRAC } 01)\end{array}$ & -310739 & -310587 & -152 \\
\hline INA & $\begin{array}{l}P-1 \text { form } \\
\text { (ISNICA) }\end{array}$ & -210518 & -210359 & -159 \\
\hline 3,5-DHBA & $\begin{array}{l}C 2 / \mathrm{c} \text { form } 2 \\
\text { (WUYPOW01) }\end{array}$ & -285351 & -285177 & -174 \\
\hline $\mathrm{SA} * \mathrm{H}_{2} \mathrm{O}$ & $\begin{array}{l}P 2_{1} / \mathrm{c} \text { form } \\
(\mathrm{SANACM})\end{array}$ & -165425 & -165194 & -231 \\
\hline SA & $\begin{array}{l}P \mathrm{ca} 2_{1} \text { form } \\
\text { AFAZEM }\end{array}$ & -285310 & -284912 & -397 \\
\hline
\end{tabular}

\section{References}

${ }^{1}$ W. Cai, A. Katrusiak. Pressure effects on H-ordering in hydrogen bonds and interactions in benzoic acid. CrystEngComm 2012, 14, 4420-4424.

${ }^{2}$ N. Okabe, H. Kyoyama. 2,3-Di-hydroxy-benzoic acid. Acta Cryst. 2001, E57, o1224-o1226.

${ }^{3}$ B. Sarma, P. Sanphui, A. Nangia. Polymorphism in Isomeric Dihydroxybenzoic Acids. Cryst. Growth Des. 2010, 10, 2388-2399.

${ }^{4}$ D. E. Braun, P. G. Karamertzanis, J.-B. Arlin, A. J. Florence, V. Kahlenberg, D. A. Tocher, U. J. Griesser, S. L. Price. Solid-State Forms of $\beta$-Resorcylic Acid: How Exhaustive Should a Polymorph Screen Be? Cryst. Growth Des. 2011, 11, 210-220.

5 A. Parkin, M. Adam, R. I. Cooper, D. S. Middlemiss, C. C. Wilson. Structure and hydrogen bonding in 2,4dihydroxybenzoic acid at 90, 100, 110 and $150 \mathrm{~K}$; a theoretical and single-crystal X-ray diffraction study. Acta Cryst. 2007, B63, 303-308.

${ }^{6}$ D. E. Braun, P. G. Karamertzanis, S. L. Price. Which, if any, hydrates will crystallise? Predicting hydrate formation of two dihydroxybenzoic acids. Chem. Commun. 2011, 47, 5443-45445.

${ }^{7}$ B. Sridhar. Synthon preference in a hydrated [beta]-resorcylic acid structure and its cocrystal with thymine. Acta Cryst. 2015, C71, 1042-1047.

${ }^{8}$ D. E. Cohen, J. B. Benedict, B. Morlan, D. T. Chiu, B. Kahr. Dyeing Polymorphs: The MALDI Host 2,5Dihydroxybenzoic Acid. Cryst. Growth Des. 2007, 7, 492-495.

${ }^{9}$ M. Gdaniec, M. Gilski, G. S. Denisov. $\gamma$-Resorcylic acid, its monohydrate and its pyridinium complex. Acta Cryst. C 1994, C50, 1622-1626.

${ }^{10}$ L. R. MacGillivray, M. J. Zaworotko. Crystal and molecular structure of 2,6-dihydroxybenzoic acid. J. Chem. Cryst. 1994, 24, 703-705.

11 Y. Wang, Y. Chen, P. Zhu, Y. Bao, C. Xie, J. Gong, X. Jiang, B. Hou, W. Chen. Measurement and Correlation of the Solubility of 2,6-Dihydroxybenzoic Acid in Alcohols and Binary Solvents. J. Chem. Eng. Data 2017, 62, 3009-3014.

12 V. Horneffer, K. Dreisewerd, H.-C. Lüdemann, F. Hillenkamp, M. Läge, K. Strupat. Is the incorporation of analytes into matrix crystals a prerequisite for matrix-assisted laser desorption/ionization mass spectrometry? A study of five positional isomers of dihydroxybenzoic acid. Int. J. Mass Spectrom. Ion. Process. 1999, 185, 859870.

${ }^{13}$ S. W. Ng. A triclinic modification of 3,4-dihy-droxy-benzoic acid monohydrate. Acta Cryst. 2011, E67, o2476.

${ }^{14}$ S. Varughese, G. R. Desiraju. Using Water as a Design Element in Crystal Engineering. Host-Guest Compounds of Hydrated 3,5-Dihydroxybenzoic Acid. Cryst. Growth Des. 2010, 10, 4184-4196.

${ }^{15}$ E. A. Heath, P. Singh, Y. Ebisuzaki Structure of p-hydroxybenzoic acid and p-hydroxybenzoic acid-acetone complex (2/1). Acta Cryst. 1992, C48, 1960-1965.

${ }^{16}$ J. J. Du, S. A. Stanton, P. A. Williams, J. A. Ong, P. W. Groundwater, J. Overgaard, J. A. Platts, D. E. Hibbs. Using Electron Density to Predict Synthon Formation in a 4-Hydroxybenzoic Acid: 4,4'-Bipyridine Cocrystal. Cryst. Growth Des. 2018, 18, 1786-1798. 
${ }^{17}$ T.-J. Hsieh, C.-C. Su, C.-Y. Chen, C.-H. Liou, L.-H. Lu. Using experimental studies and theoretical calculations to analyze the molecular mechanism of coumarin, p-hydroxybenzoic acid, and cinnamic acid. J. Mol. Struct. 2005, 741, 193-199.

${ }^{18}$ S. Athimoolam, S. Natarajan. 4-Carboxy-anilinium (2R,3R)-tartrate and a redetermination of the [alpha]polymorph of 4-amino-benzoic acid. Acta Cryst. 2007, C63, o514-0517.

${ }^{19}$ M. R. Ward, S. Younis, A. J. Cruz-Cabeza, C. L. Bull, N. P. Funnell, I. D. H. Oswald. Discovery and recovery of delta p-aminobenzoic acid. CrystEngComm 2019, 21, 2058-2066.

20 R. Benali-Cherif, R. Takouachet, E.-E. Bendeif, N. Benali-Cherif. The structural properties of a noncentrosymmetric polymorph of 4-amino-benzoic acid. Acta Cryst. 2014, C70, 323-325.

${ }^{21}$ R.-W. Jiang, D.-S. Ming, P. P. H. But, T. C. W. Mak Gallic acid monohydrate. Acta Cryst. 2000, C56, 594-595.

${ }^{22}$ N. Okabe, H. Kyoyama, M. Suzuki. Gallic acid monohydrate. Acta Cryst. 2001, E57, o764-o766.

${ }^{23}$ H. D. Clarke, K. K. Arora, L. Wojtas, M. J. Zaworotko. Polymorphism in Multiple Component Crystals: Forms III and IV of Gallic Acid Monohydrate. Cryst. Growth Des. 2011, 11, 964-966.

${ }^{24}$ D. E. Braun, R. M. Bhardwaj, A. J. Florence, D. A. Tocher, S. L. Price. Complex Polymorphic System of Gallic Acid-Five Monohydrates, Three Anhydrates, and over 20 Solvates. Cryst. Growth Des. 2013, 13, 19-23.

${ }^{25}$ A. A. Hoser, I. Sovago, A. Lanzac, A. Ø. Madsen. A crystal structure prediction enigma solved: the gallic acid monohydrate system - surprises at 10 K. Chem. Commun. 2017, 53, 925-928.

${ }^{26}$ B. Kozlevčar, D. Odlazek, A. Golobič, A. Pevec, P. Strauch, P. Šegedin. Complexes with lignin model compound vanillic acid. Two different carboxylate ligands in the same dinuclear tetracarboxylate complex [Cu2(C8H7O4)2(O2CCH3)2(CH3OH)2]. Polyhedron 2006, 25, 1161-1166.

${ }^{27} \mathrm{~J}$. N. Low, C. Glidewell. A quasi-diamondoid hydrogen-bonded framework in an-hydro-us sulfanilic acid. Acta Cryst. 2002, C58, o209-0211.

${ }^{28}$ S. K. Callear, M. B. Hursthouse. University of Southampton, Crystal Structure Report Archive 2008, 552, doi: $10.5258 /$ ecrystals/552

${ }^{29}$ A. I. M. Rae. E. N. Maslen. The crystal structure of sulphanilic acid monohydrate. Acta Cryst. 1962, 15, 12851291.

${ }^{30}$ A. Banu, G. M. Golzar Hossain. A new polymorph of sulfanilic acid monohydrate. Acta Cryst. 2006, E62, o2252-o2253.

${ }^{31}$ Q. Wang, CSD Communication 2016

${ }^{32}$ S. Langenstück, C. Zhao, U. Englert. The ephemeral dihydrate of sulfanilic acid. Acta Cryst. 2018, C74, 7-12.

${ }^{33}$ F. Takusagawa, A. Shimada. Isonicotinic acid. Acta Cryst. 1976, B32, 1925-1927.

${ }^{34} \mathrm{~J}$. Li, S. A. Bourne, M. R. Caira. New polymorphs of isonicotinamide and nicotinamide. Chem. Commun. 2011, 47, 1530-1532.

${ }^{35}$ K. S. Eccles, R. E. Deasy, L. Fábián, D. E. Braun, A. R. Maguire, S. E. Lawrence. Expanding the crystal landscape of isonicotinamide: concomitant polymorphism and co-crystallisation. CrystEngComm 2011, 13, 69236925.

${ }^{36}$ A. I. Vicatos, M. R. Caira. A new polymorph of the common coformer isonicotinamide. CrystEngComm 2019, 21, 843-849.

${ }^{37}$ N. B. Báthori, A. Lemmerer, G. A. Venter, S. A. Bourne, M. R. Caira. Pharmaceutical Co-crystals with Isonicotinamide-Vitamin B3, Clofibric Acid, and Diclofenac-and Two Isonicotinamide Hydrates. Cryst. Growth Des. 2011, 11, 75-87.

${ }^{38}$ N. Shankland, A. J. Florence, P. J. Cox, D. B. Sheen, S. W. Love, N. S. Stewart, C. C. Wilson. Crystal morphology of ibuprofen predicted from single-crystal pulsed neutron diffraction data. Chem. Commun. 1996, 855-856.

39 P. Derollez, E. Dudognon, F. Affouard, F. Danède, N. T. Correia, M. Descamps. Ab initio structure determination of phase II of racemic ibuprofen by X-ray powder diffraction. Acta Cryst. 2010, B66, 76-80.

${ }^{40}$ M. N. Frey, T. F. Koetzle, M. S. Lehmann, W. C. Hamilton. Precision neutron diffraction structure determination of protein and nucleic acid components. X. A comparison between the crystal and molecular structures of L-tyrosine and L-tyrosine hydrochloride. J. Chem. Phys. 1973, 58, 2547.

${ }^{41}$ N. Tumanova, N. Tumanov, K. Robeyns, F. Fischer, L. Fusaro, F. Morelle, V. Ban, G. Hautier, Y. Filinchuk, J. Wouters, T. Leyssens, F. Emmerling. Opening Pandora's Box: Chirality, Polymorphism, and Stoichiometric Diversity in Flurbiprofen/Proline Cocrystals. Cryst. Growth Des. 2018, 18, 954-961.

42 J. Janczak, P. Luger. L-Proline Monohydrate at 100 K. Acta Cryst 1997, C53, 1954-1956.

${ }^{43}$ T. C. Lewis, D. A. Tocher, S. L. Price. An Experimental and Theoretical Search for Polymorphs of Barbituric Acid: The Challenges of Even Limited Conformational Flexibility. Cryst. Growth Des. 2004, 4, 979-987.

${ }^{44}$ D. M. Többens, J. Glinneman, M. R. Chierotti, J. van de Streek, D. Sheptyakov. On the high-temperature phase of barbituric acid. CrystEngComm 2012, 14, 3046-3055. 
${ }^{45}$ M. U. Schmidt, J. Brüning, J. Glinnemann, M. W. Hützler, P. Mörschel, S. N. Ivashevskaya, J. van de Streek, D. Braga, L. Maini, M. R. Chierotti, R. Gobetto. The Thermodynamically Stable Form of Solid Barbituric Acid: The Enol Tautomer. Angew. Chem. Int. Ed. 2011, 50, 7924-7926.

${ }^{46}$ G. S. Nichol, W. Clegg. A variable-temperature study of a phase transition in barbituric acid dihydrate. Acta Cryst. 2005, B61, 464-472. 